\title{
Experimental Investigation of a Flexible Wing with a Variable Camber Continuous Trailing Edge Flap Design
}

\author{
Nhan Nguyen * \\ NASA Ames Research Center, Moffett Field, CA 94035 \\ Nathan Precup ${ }^{\dagger}$ \\ University of Washington, Seattle, WA 98195 \\ James Urnes, Sr. $\ddagger$ \\ Boeing Research \& Technology, St. Louis, MO 63134 \\ Chester Nelson $\S$ \\ Boeing Commercial Airplanes, WA \\ Sonia Lebofsky II \\ Stinger Ghaffarian Technologies Inc., Moffett Field, CA 94035 \\ Eric Ting $\|$ \\ Stinger Ghaffarian Technologies Inc., Moffett Field, CA 94035 \\ Eli Livne ** \\ University of Washington, Seattle, WA 98195
}

\begin{abstract}
This paper presents experimental results of a flexible wing wind tunnel model with a variable camber continuous trailing edge flap (VCCTEF) design for drag minimization, tested at the University of Washington Aeronautical Laboratory (UWAL). The wind tunnel test was designed to explore the relative merit of the VCCTEF concept for improved cruise efficiency through the use of low-cost aeroelastic model test techniques. The flexible wing model is a $10 \%$-scaled model of a typical transport wing and is constructed of woven fabric composites and foam core. The wing structural stiffness in bending is tailored to be half of the stiffness of a Boeing 757-era transport wing, while the torsional stiffness is about the same. This stiffness reduction results in a wing tip deflection of about $10 \%$ of the wing semi-span. The VCCTEF is a multi-segment flap design having three chordwise camber segments and five spanwise flap sections for a total of 15 individual flap elements. The three chordwise camber segments can be positioned appropriately to create a desired trailing edge camber. Elastomeric material is used to cover the gaps in between the spanwise flap sections, thereby creating a continuous trailing edge. Wind tunnel data indicate a high degree of data correlation and repeatability. The VCCTEF can achieve a drag reduction of up to $6.31 \%$ and an improvement in the lift-to-drag ratio $(L / D)$ of up to $4.85 \%$. The paper also presents two methods for estimating the lift coefficient of a rigid wing using a dynamic pressure correction and an aeroelastic deflection correction. Both methods provide good estimates of the rigid-wing lift coefficient.
\end{abstract}

\section{Introduction}

The aircraft industry has been responding to the need for energy-efficient aircraft by redesigning airframes to be aerodynamically efficient, employing light-weight materials for aircraft structures and incorporating more energy-efficient aircraft engines. Reducing airframe operational empty weight (OEW) using advanced composite materials is one of the major considerations for improving energy efficiency. Modern light-weight materials can provide less structural rigidity while maintaining sufficient

\footnotetext{
*NASA Ames Research Center, Research Scientist, AIAA Associate Fellow, nhan.t.nguyen@nasa.gov

†University of Washington Aeronautical Laboratory, Operations Manager, nprecup@u.washington.edu

${ }^{\ddagger}$ Boeing Research \& Technology, Program Manager, james.m.urnes-sr@boeing.com

${ }^{\S}$ Boeing Commercial Airplanes, Boeing Technical Fellow, chester.p.nelson@ boeing.com

IIStinger Ghaffarian Technologies Inc., NASA Ames Research Center, Research Engineer, sonia.lebofsky@nasa.gov

" Stinger Ghaffarian Technologies Inc., NASA Ames Research Center, Research Engineer, eric.b.ting@nasa.gov

** University of Washington, Endowed Boeing Professor of Aeronautics and Astronautics, eli@aa.washington.edu
} 
load-carrying capacity. As structural flexibility increases, aeroelastic interactions with aerodynamic forces and moments can alter aircraft aerodynamics significantly, thereby potentially degrading aerodynamic efficiency.

Under the Fundamental Aeronautics Program in the NASA Aeronautics Research Mission Directorate, the Fixed Wing Project is conducting multidisciplinary research to investigate advanced concepts and technologies for future aircraft systems. A NASA study entitled "Elastically Shaped Future Air Vehicle Concept" was conducted in $2010^{1,2}$ to examine new concepts that can enable active control of wing aeroelasticity to achieve drag reduction. This study showed that highly flexible wing aerodynamic surfaces can be elastically shaped in-flight by active control of wing twist and vertical deflection in order to optimize the local angles of attack of wing sections to improve aerodynamic efficiency through drag reduction during cruise and enhanced lift performance during take-off and landing.

The study shows that active aeroelastic wing shaping control can have a potential drag reduction benefit. Conventional flap and slat devices inherently generate drag as they increase lift. The study shows that conventional flap and slat systems are not aerodynamically efficient for use in active aeroelastic wing shaping control for drag reduction. A new flap concept, referred to as the Variable Camber Continuous Trailing Edge Flap (VCCTEF) system, was conceived by NASA to address this need. ${ }^{1}$ Initial study results indicate that, for some applications, the VCCTEF system may offer a potential pay-off in drag reduction that could provide significant fuel savings. In order to realize the potential benefit of drag reduction by active span-load and aeroelastic wing shaping control while meeting all other performance requirements, the approach for high lift devices needs to be considered as part of the wing shaping control strategy.

NASA and Boeing are currently conducting a joint study to develop the VCCTEF further under the research element Adaptive Aeroelastic Shape Control (AASC) within the Fixed Wing Project. ${ }^{3,4}$ This study is built upon the development of the VCCTEF system for the NASA Generic Transport Model (GTM), which is essentially based on the Boeing 757 airframe, ${ }^{5}$ employing light-weight Shape Memory Alloy (SMA) technology for actuation and three separate chordwise segments shaped to provide a variable camber to the flap. This cambered flap has potential for drag reduction as compared to a conventional straight, plain flap. The flap is also made up of individual 2-foot spanwise sections, which enable different flap settings at each flap spanwise position. This results in the ability to control the wing twist shape as a function of span, resulting in a change to the wing twist to establish the best lift-to-drag ratio $(L / D)$ at any aircraft gross weight or mission segment. Wing twist on traditional commercial transport designs is dictated by the aeroelastic deflection of a fixed "jig twist" shape applied at manufacture. The design of this jig twist is set for one cruise configuration, usually for a $50 \%$ fuel loading or mid-point on the gross weight schedule. The VCCTEF offers different wing twist settings, hence different spanwise loadings, for each gross weight condition and also different settings for climb, cruise and descent, a major factor in obtaining best L/D conditions.

The second feature of VCCTEF is a continuous trailing edge flap. The individual 2-foot spanwise flap sections are connected with a flexible covering, so no breaks can occur in the flap planforms, thus reducing drag by eliminating these breaks in the flap continuity, which otherwise would generate vorticity that results in a drag increase and also contributes to airframe noise. This continuous trailing edge flap design combined with the flap camber result in lower drag increases during flap deflections. In addition, it also offers a potential noise reduction benefit.

The VCCTEF serves multiple functions as:

- A wing shaping control device to twist the flexible wing and change the span-load distribution to obtain changes in lift-to-drag ratios that will reduce cruise drag throughout the flight envelope.

- A high-lift device for take-off, climb-out, let-down and final approach by using the full span cambered flap.

- A full span roll control effector in lieu of traditional ailerons using the aft section of the cambered flap.

- An aeroservoelastic (ASE) control device to compensate for reduced flutter margins of flexible wings.

\section{Description of the VCCTEF System}

The GTM (Generic Transport Model) is a 5.5\%-scaled research transport platform that includes a wind tunnel model and a remotely piloted vehicle, as shown in Fig. 1. It represents a notional single-aisle, mid-size, 200-passenger transport aircraft generically approximating a Boeing 757. The full-scale geometry of the GTM is obtained by scaling up the 5.5\%-scaled geometry. Figure 2 is an illustration of the GTM planform. The GTM had been extensively tested in the 14-foot-by-22foot wind tunnel at NASA Langley Research Center. Thus, wind tunnel test data are available that can be used to validate computational models. 


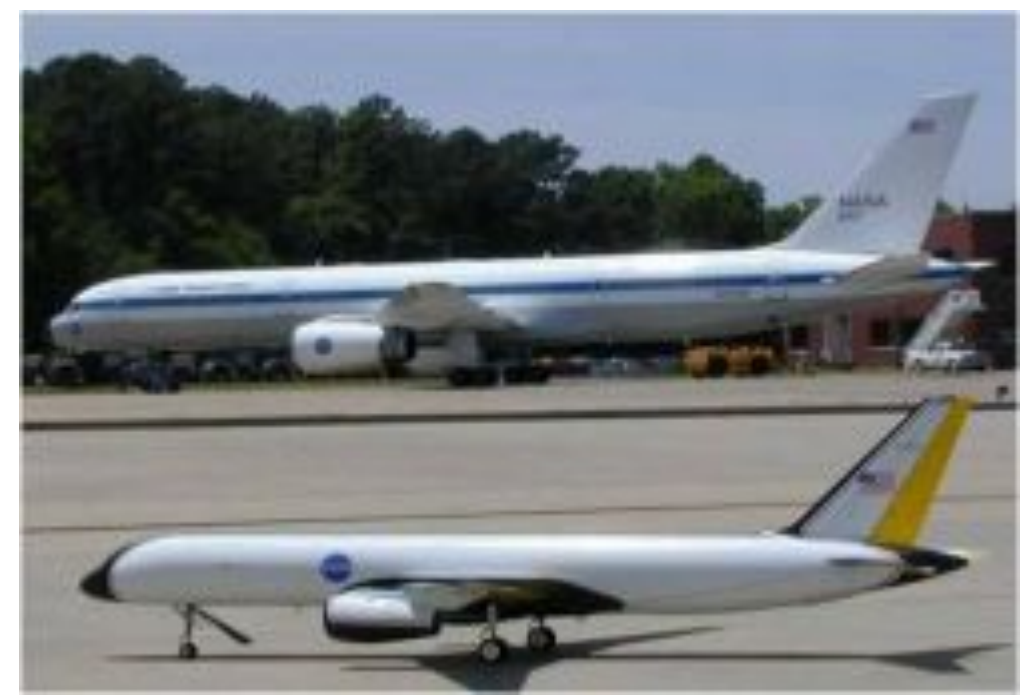

Fig. 1 - Generic Transport Model (GTM) and Remotely Piloted Vehicle at NASA Langley

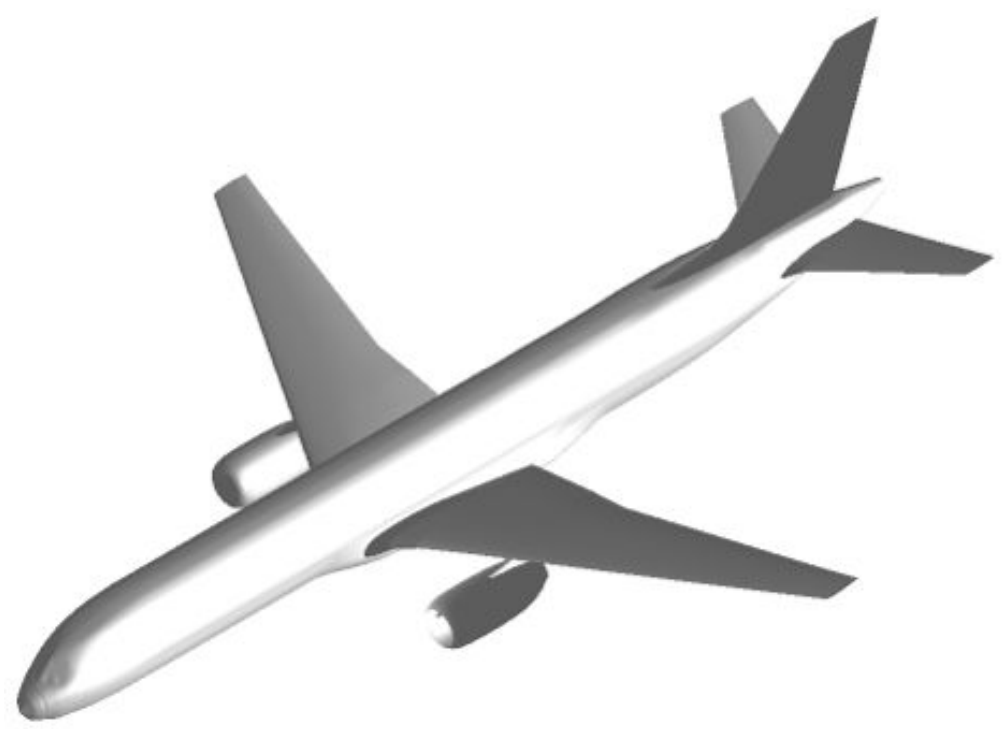

Fig. 2 - GTM Planform

The aircraft has a mid-cruise weight of 210,000 lbs for a typical operating load (gear up, flap up) that includes cargo, fuel, and passengers. Fuel weighs about 50,000 lbs for a range of about 3,000 nautical miles. At the design cruise condition of Mach 0.8 at $36,000 \mathrm{ft}$, the design lift coefficient is 0.51 .

To enable effective wing shaping control, the GTM wings are modeled as modern transport wings with a high degree of flexibility, similar to estimated flexibility distributions on state-of-the-art passenger aircraft wings. The wing bending stiffness is tailored to achieve a $10 \%$ wing tip deflection, which results in a bending stiffness about half that of older-generation transport wings, while the torsional stiffness is about the same. This $10 \%$ wing tip deflection is about the same as that of a modern composite high-aspect-ratio wing design in modern transport aircraft such as the Boeing 787.

The VCCTEF is divided into 14 sections attached to the outer wing and 3 sections attached to the inner wing, as shown in Fig. 3. ${ }^{4}$ Each 24-inch section has three camber flap segments that can be individually commanded. These camber flaps are joined to the next section by a flexible and supported material (shown in blue) installed with the same shape as the camber and thus providing continuous flaps throughout the wing span with no drag producing gaps. 


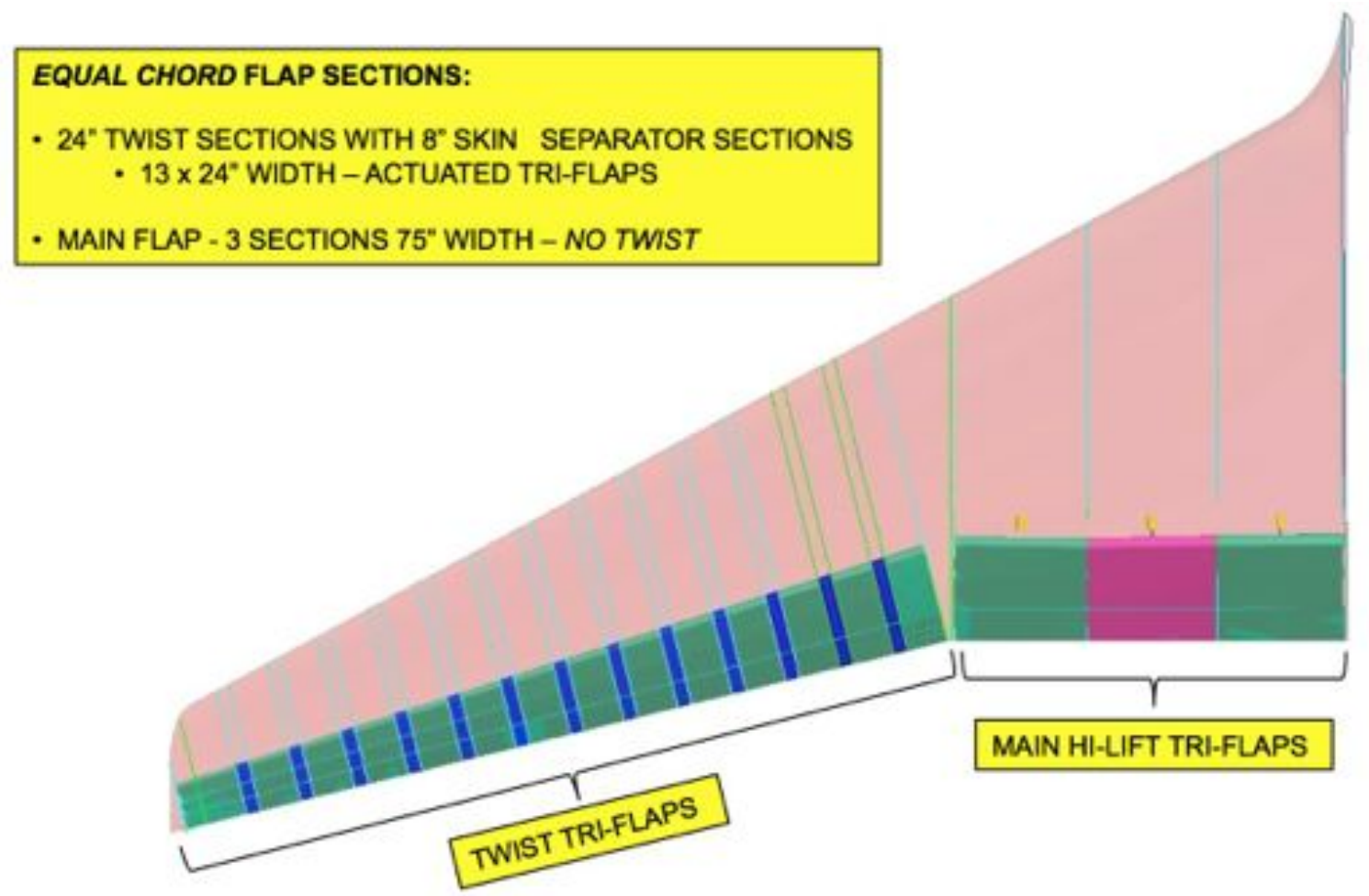

Fig. 3 - Wing Configured with the Variable Camber Continuous Trailing Edge Flap

A major goal of the program is to develop a light-weight flap control system that has a significant weight advantage as compared to current flap screw-jack actuators. Hydraulic, electric and Shape Memory Alloy (SMA) torque rod actuation were evaluated with the result that the SMA actuation has the best weight advantage, as shown in Fig. $4 .{ }^{4}$ Moreover, the use of hinge line actuation eliminates the large and heavy external mounted actuators, and permits all actuators to be interior to the wing and flap mold lines, thus contributing to the overall drag reduction goal.

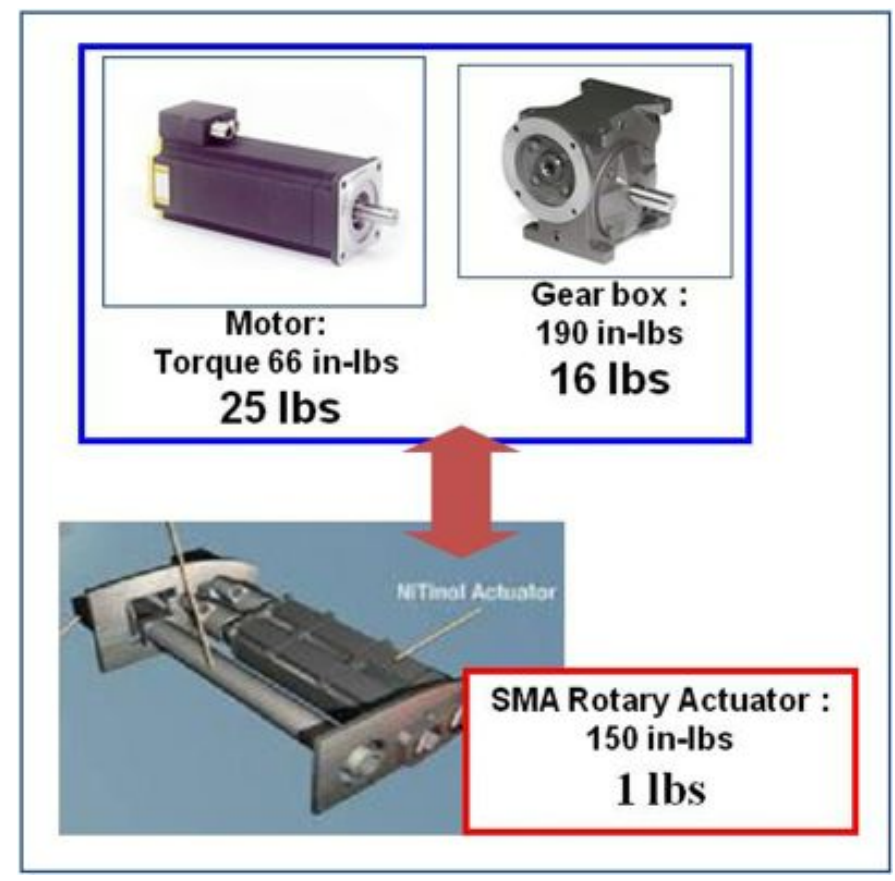

Fig. 4 - Shape Memory Alloy Actuators Have a Low Weight Compared to Electric Motor Actuators

Figure 5 shows a schematic representation of three of the outer wing flap sections, each having three camber components. ${ }^{4}$ 


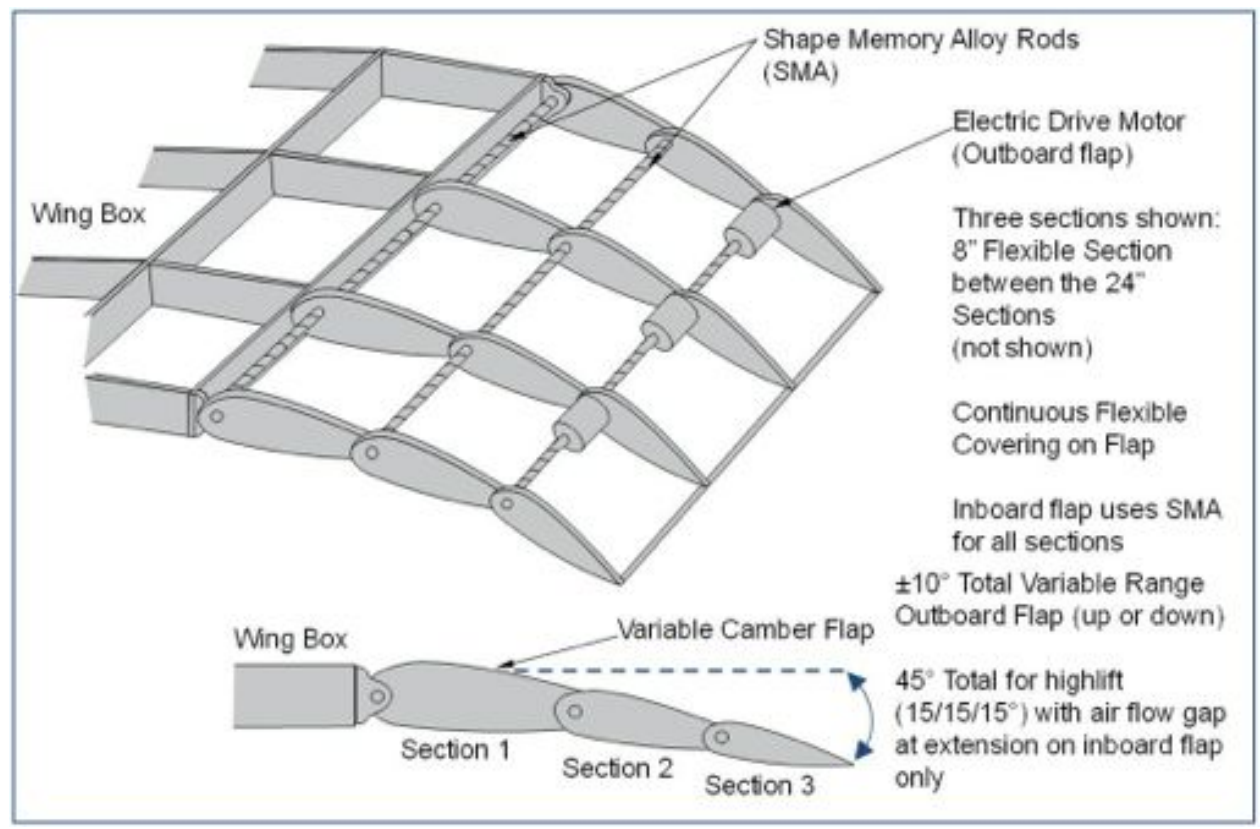

Fig. 5 - The Variable Camber Flap Control uses Shape Memory Alloy Torque Rod and Electric Drive Actuation

SMA actuators drive the first and second camber flap segments and a faster acting electric actuator drives the third camber flap segment. SMA actuators can deliver large hinge moments, but generally move at a slow rate. The outer wing flap uses the full-span third camber segment as a roll command effector and as a control device for suppressing aeroelastic wing structural dynamic modes, both requiring high rates which can be met by electric actuators. Figure 6 shows an SMA actuator. ${ }^{4}$

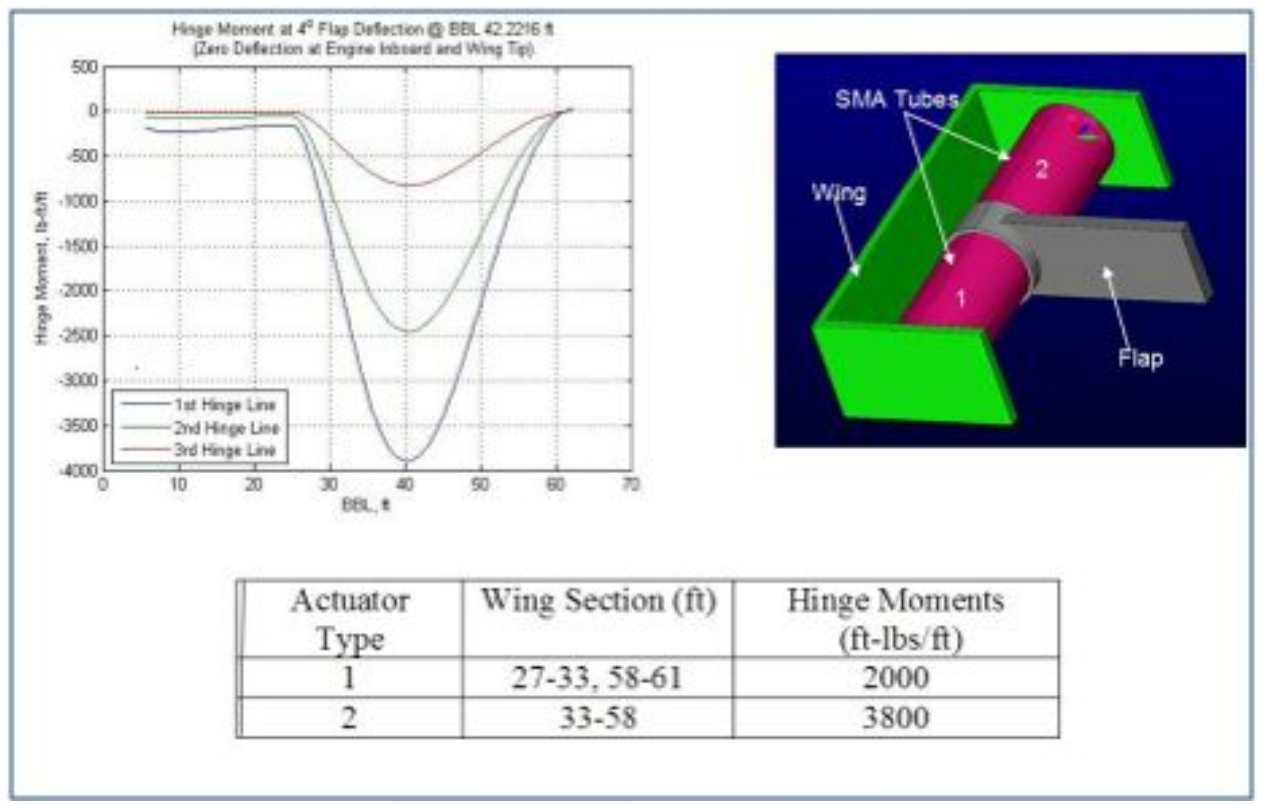

Fig. 6 - Shape Memory Alloy Actuators can Meet the VCCTEF Hinge Moment Requirements

Using the camber positioning, a full-span, low-drag, high-lift configuration can be activated that has no drag producing gaps and a low flap noise signature. This is shown in Fig. 7. To further augment lift, a slotted flap configuration is formed by an air passage between the wing and the inner flap that serves to improve airflow over the flap and keep the flow attached. This air passage appears only when the flaps are extended in the high lift configuration. 


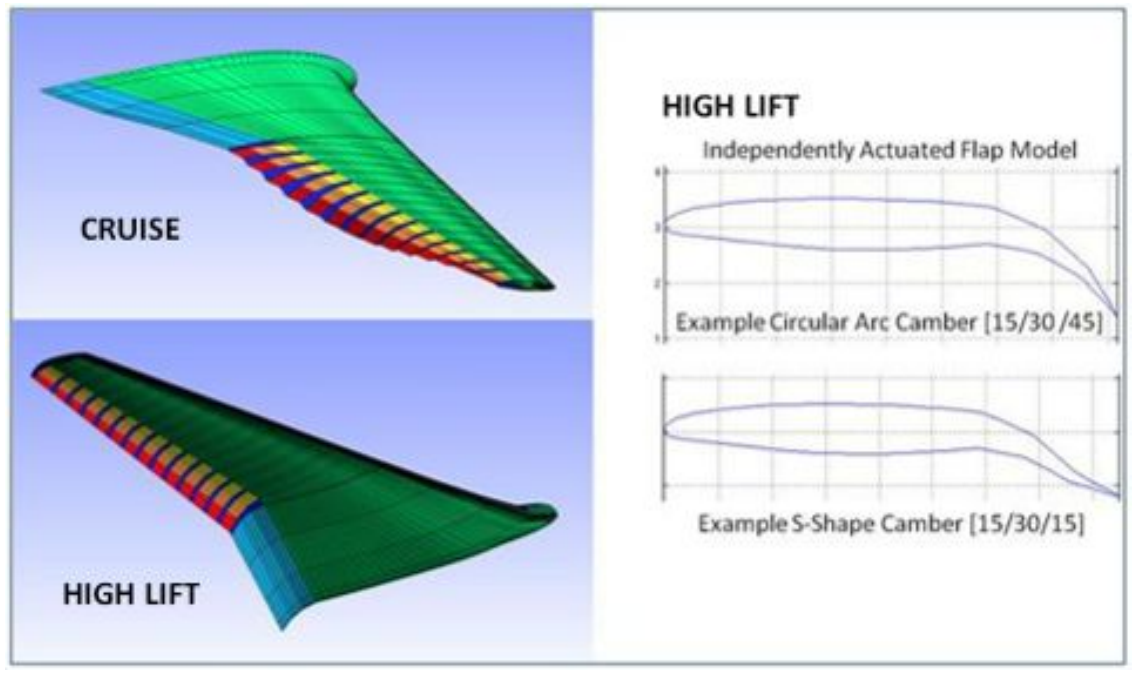

Fig. 7 - Cruise and High Lift VCCTEF Configurations

In the high-lift configuration, the outer wing flap uses the third camber section for roll control, as shown in Fig. 8. This provides rolling moment that is equivalent to aileron control. It is somewhat similar to deflecting the ailerons in a droop position to act as flaps, a common procedure used on tactical aircraft and on some transport aircraft.

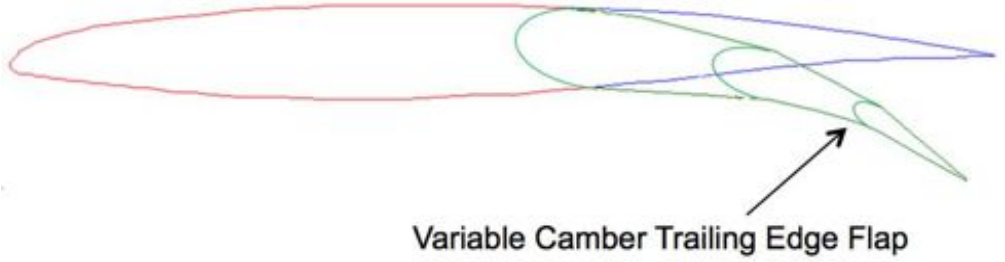

Fig. 8 - Variable Camber Flap

The high-lift configuration distributes the required flap hinge moment throughout the span of the wing while using actuation components that are all located interior to the wing and flap. This can be achieved by the use of SMA hinge line torque rods, sized to meet the hinge moment requirements at each spanwise location on the wing.

Figure 9 illustrates the GTM equipped with the VCCTEF for wing shaping control. By actively shaping the wing aerodynamic surface using the VCCTEF, optimal aerodynamic performance could potentially be realized at any point in the flight envelope. This is akin to the mission-adaptive wing technology which was demonstrated on F-111 in the 1990's. The VCCTEF relies on two mechanisms to improve aerodynamic performance: 1) wing twist optimization for flexible wing design, and 2) variable camber and continuous trailing edge for improved aerodynamics. This fixed-wing technology may be referred to as Performance Adaptive Aeroelastic Wing (PAAW) technology. This technology would enable modern high-aspect ratio wings with a high degree of flexibility to adaptively change shapes in-flight for real-time drag optimization, while at the same time satisfying operational constraints such as structural load limitations, flutter margins, gust and maneuver load responses, and others by active aeroservoelastic controls and or passive aeroelastic tailoring.

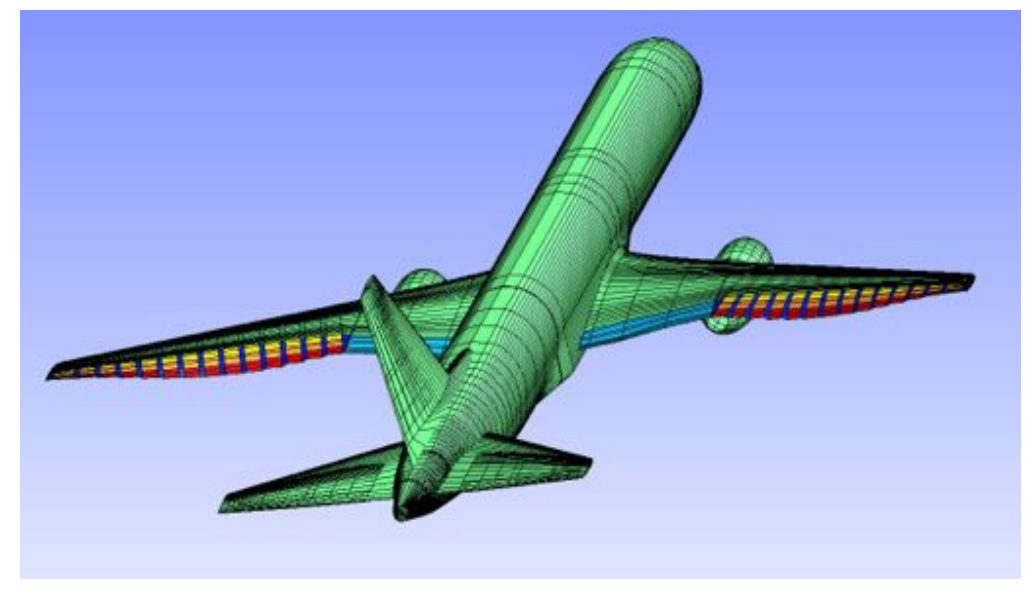


Fig. 9 - GTM with VCCTEF

\section{Wind Tunnel Model of Flexible Wing}

For exploratory assessment of the aerodynamic potential of the VCCTEF concept, a 10\%-scaled aeroelastic model of a softened Boeing 757-based GTM wing was constructed for a wind tunnel experimental investigation in the University of Washington Aeronautical Laboratory (UWAL). The semi-span of the model is 73.29 inches, as shown in Fig. 10. The model is constructed of woven fabric composites skin and extruded polystyrene foam core. The composite laminates and extruded polystyrene foam core are structurally tailored to attain half of the bending stiffness of the scaled baseline GTM wing stiffness while keeping torsional stiffness about the same. This tailored stiffness is to achieve a $10 \%$ wing tip deflection. The VCCTEF parts are fabricated by 3D printing. The flap segments are mechanically interlocking aerodynamic surfaces in the chordwise direction and mate with silicone elastomer material between spanwise flap sections, as shown in Fig. 11. The flap segments are hinged at three chordwise locations and are designed to be fully adjustable. The dimensions of the VCCTEF are shown in Fig. 12.

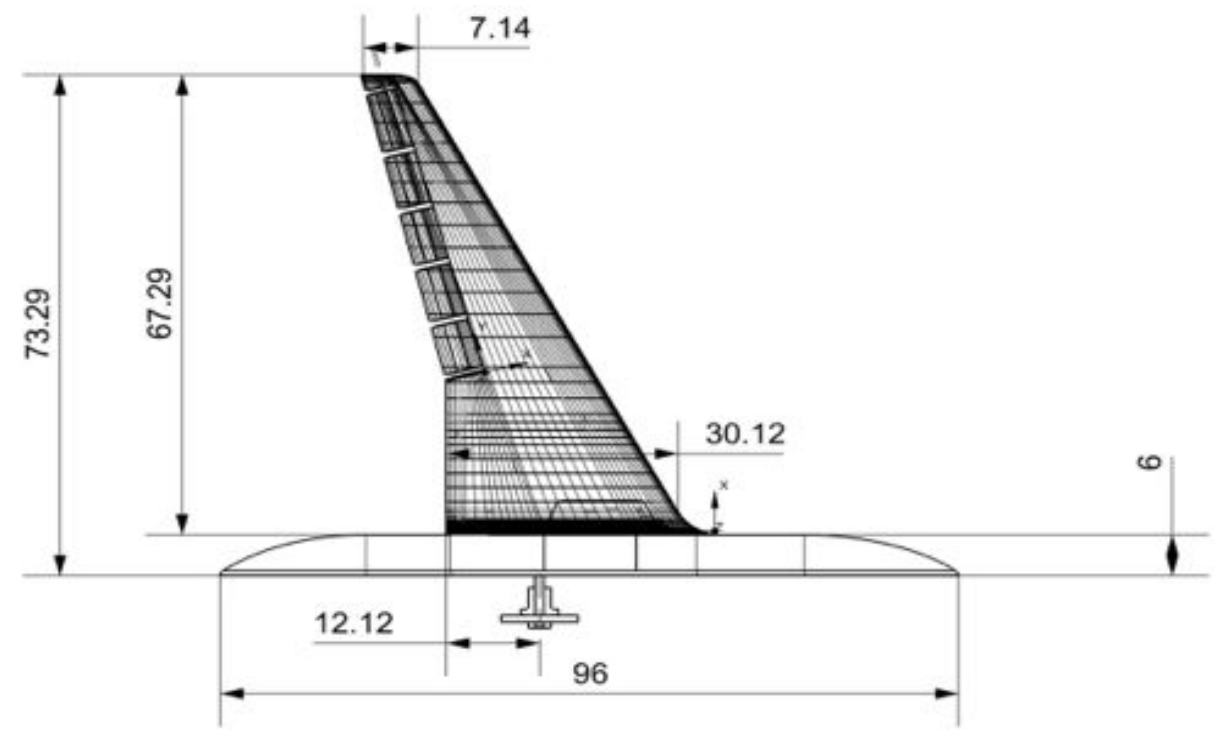

Fig. 10 - UWAL Wind Tunnel Model with VCCTEF (Courtesy of University of Washington Aeronautical Laboratory)

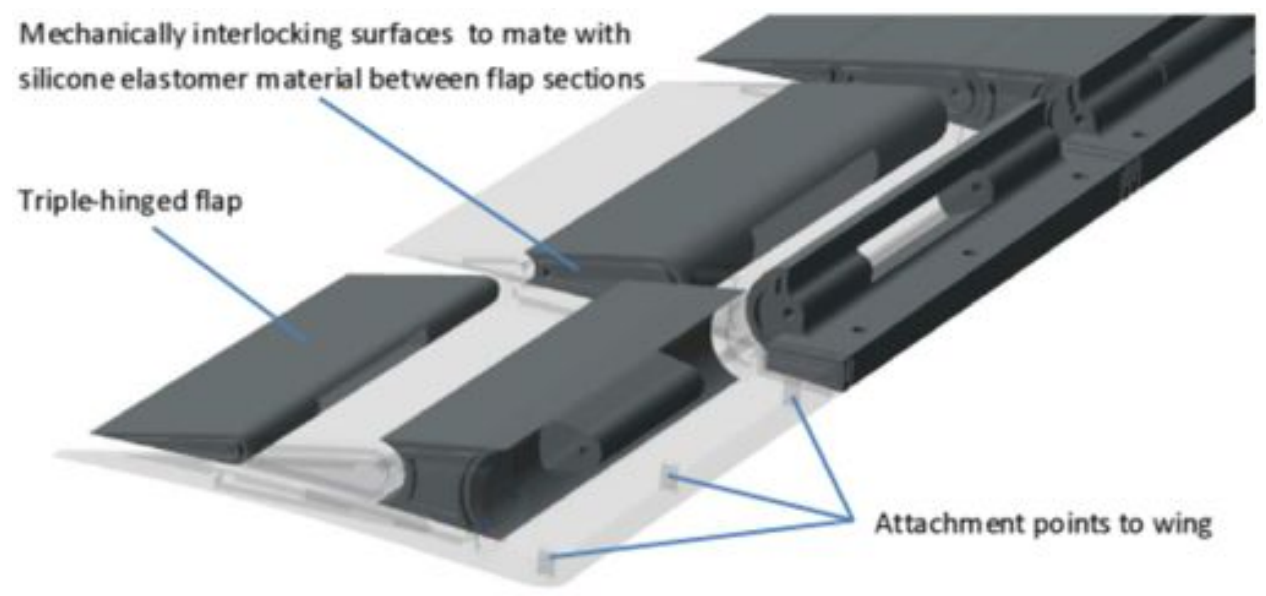

3D CAD view of VCCTEF Flap design (with segments ghosted to show internal design)

Fig. 11 - VCCTEF Construction (Courtesy of University of Washington Aeronautical Laboratory) 


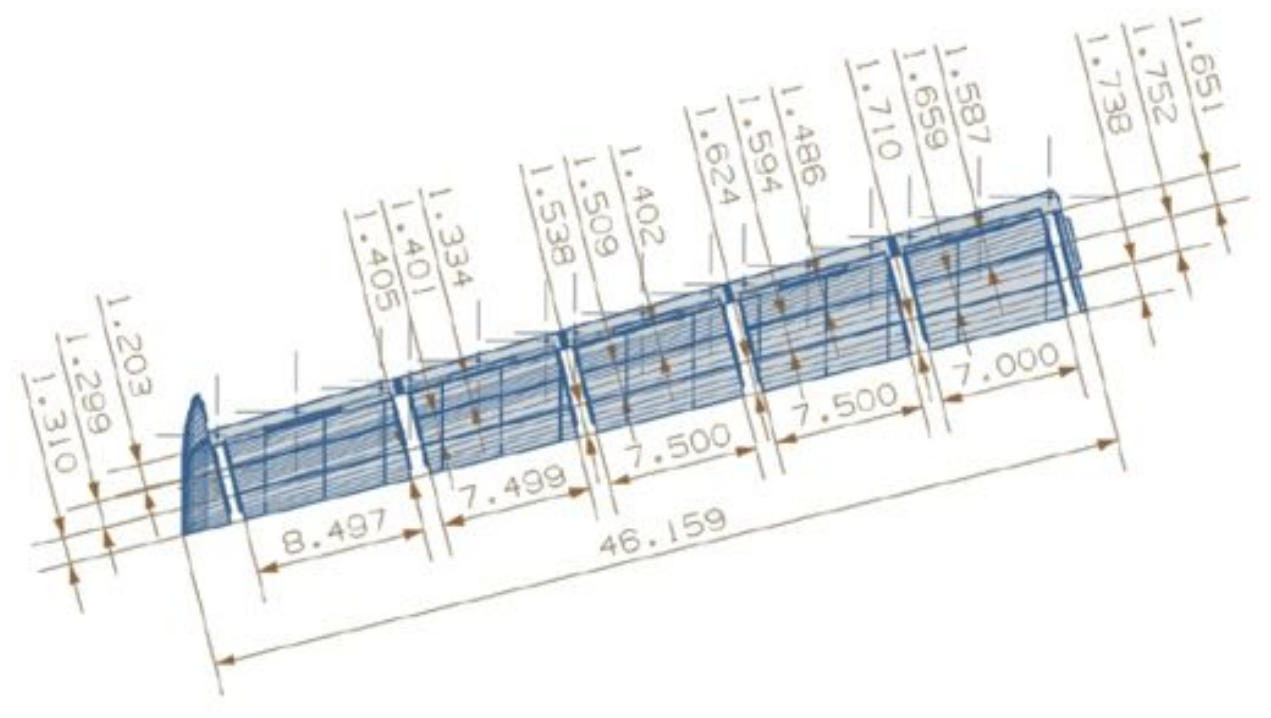

Drawing specifying planform view flap dimensions (measurements in inches)

Fig. 12 - VCCTEF Dimension (Courtesy of University of Washington Aeronautical Laboratory)

Figure 13 shows an exploded view of the UWAL wind tunnel model of the flexible wing mated to a center body fairing attached to an external floor-mounted balance.

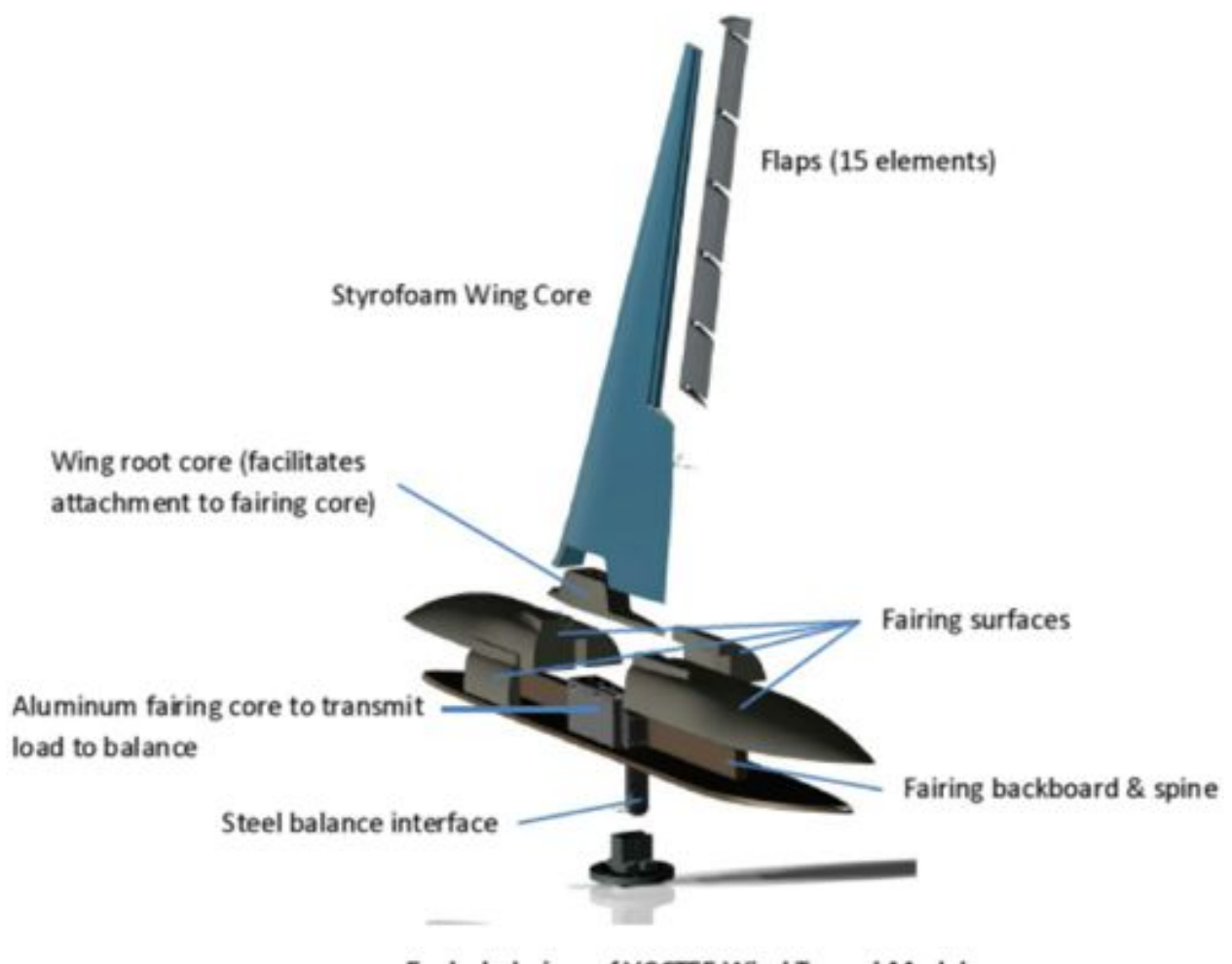

Exploded view of VCCTEF Wind Tunnel Model

Fig. 13 - Exploded View of Wind Tunnel Model (Courtesy of University of Washington Aeronautical Laboratory)

The UWAL aeroelastic wind tunnel model was built with different stiffness than the scaled GTM wing and its jig shape was not optimized for best aerodynamic performance at test conditions. The same jig shape as that of the GTM wing was used. The wash-out twist is therefore non-optimal for the model when it operates at the design lift coefficient of 0.51. A CFD optimization was conducted prior to the test to identify an optimal jig twist. However, this optimized jig twist was not incorporated into the final model fabrication due to programmatic issues.

The relevant model scaling information is given in Table 1. 


\begin{tabular}{|c|c|c|}
\hline & Full-Scale & Semi-Span Model \\
\hline \hline$M_{\infty}$ & 0.797 & 0.1162 \\
\hline$C_{L}$ & 0.51 & 0.51 \\
\hline$h, \mathrm{ft}$ & 36,000 & 0 \\
\hline$q_{\infty}, \mathrm{psf}$ & 211.09 & 20.00 \\
\hline$S / 2, \mathrm{ft}^{2}$ & 975.5 & 9.638 \\
\hline $\bar{c}, \mathrm{ft}$ & 16.6417 & 1.5963 \\
\hline$b / 2, \mathrm{ft}$ & 62.4167 & 6.1262 \\
\hline
\end{tabular}

Table 1 - Model Parameters

The wind tunnel test is designed to be an exploratory, proof-of-concept study. The objective of the wind tunnel experiment is to explore the relative merit of the VCCTEF design as a drag reduction control device, and the ability to simulate the problem in a relatively low-cost test. Lift, drag, side force, pitching moment, yawing moment, and rolling moment were recorded from the external floor-mounted balance. In addition, aeroelastic deflections of the flexible wing model were also measured by a VICON motion tracking system. The VICON system measured the three-dimensional displacement of the wind tunnel model at 54 points on the model.

To ensure that the wind tunnel model has correct aeroelastic properties, static load tests and frequency measurements were conducted. A detail three-dimensional (3D) NASTRAN model was constructed for comparison with the measurements. The 3D NASTRAN results demonstrate an excellent agreement with the static load test data and measured frequencies. A stick NASTRAN model was constructed to match the deflection information from the 3D NASTRAN model. In addition, a NASTRAN Doublet Lattice flutter analysis was performed to ensure that the wind tunnel is flutter-free in the wind tunnel. Flutter speeds were determined to be well above the test section airspeed of $39.54 \mathrm{~m} / \mathrm{sec}$.

\section{Wind Tunnel Test}

The test was conducted at a nominal dynamic pressure of 20 psf. Off-condition data and additional runs at 10, 15, 25 and $30 \mathrm{psf}$ were also collected for some cases. The nominal test section airspeed was Mach 0.1162. Figure 14 is a photograph of the flexible wing wind tunnel model in the UWAL test section.

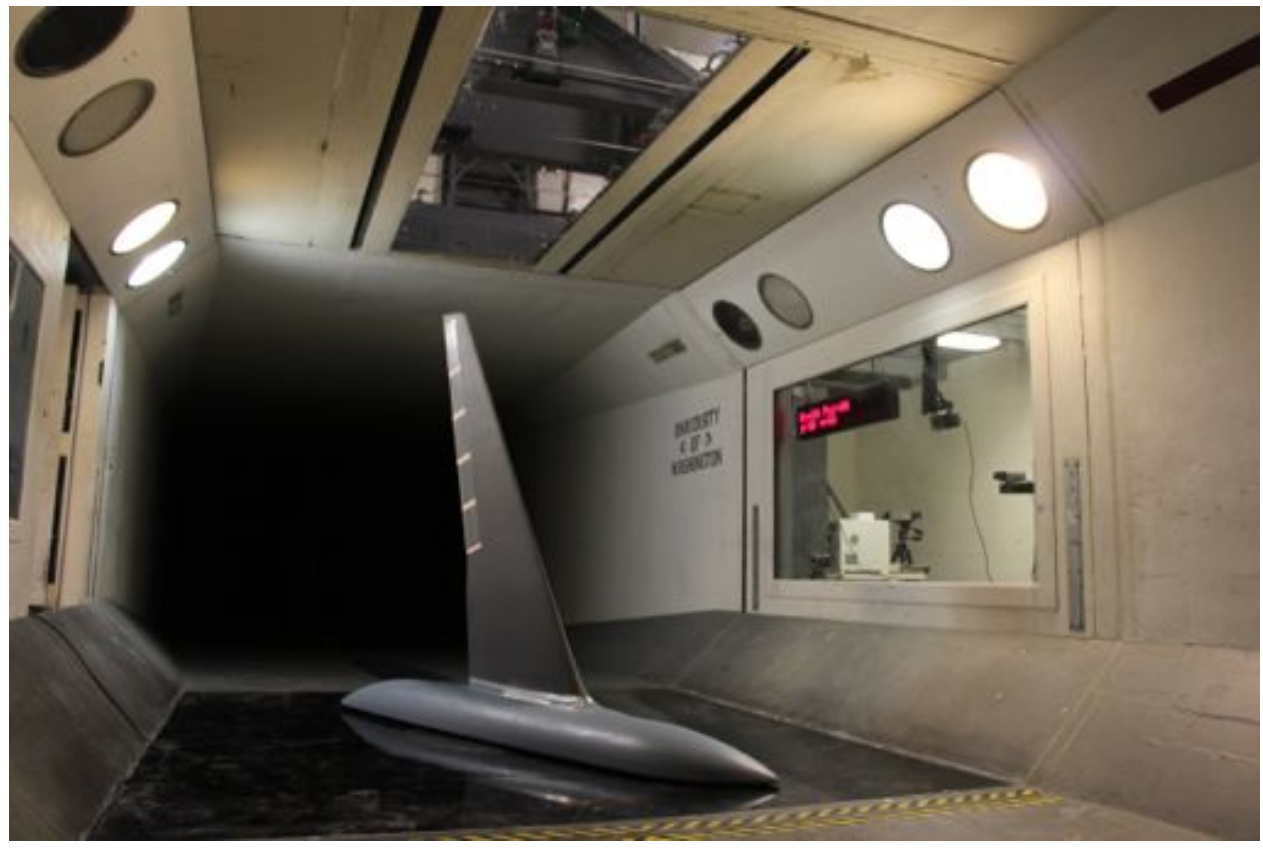

Fig. 14 - Flexible Wing Wind Tunnel Model in UWAL Test Section (Courtesy of University of Washington Aeronautical Laboratory)

The wind tunnel model was tested with a total of $13 \mathrm{VCCTEF}$ configurations ranging from zero to full deflection. These VCCTEF configurations are designated as: 
- FLAP0 - baseline zero deflection

- FLAP1 - full deflection for all flap sections

- FLAP2 - varying from a maximum deflection at the inboard and outboard flaps to a minimum deflection at the mid-span flap

- FLAP3 - varying from a minimum deflection at the inboard and outboard flaps to a maximum deflection at the mid-span flap

- FLAP4 - varying monotonically from a maximum deflection at the inboard flap to zero deflection at the outboard flap

- FLAP5 - varying monotonically from zero deflection at the inboard flap to a maximum deflection at the outboard flap

- FLAP6 - similar to FLAP4 configuration but with a smaller deflection

- FLAP7 - varying monotonically from a maximum positive deflection at the inboard flap to a negative deflection at the outboard flap

- FLAP8 - rigid-body deflection with the two outer camber segments at zero relative deflection

- FLAP9 - deflection of the trailing edge camber segments

- FLAP10 - intermediate deflection

- FLAP11 - full negative deflection

- FLAP12 - FLAP6 configuration plus a gurney flap

Figure 15 illustrates some of these flap configurations.

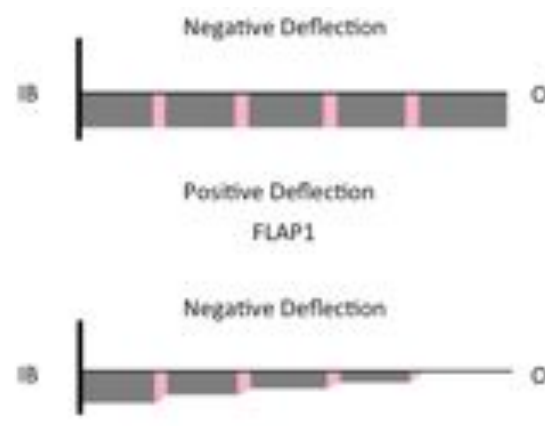

Positive Deflection

FLAPS

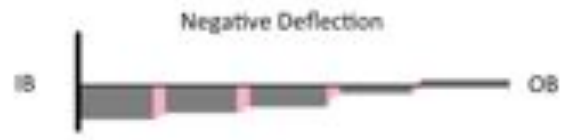

Positive Deflection

FUAP?

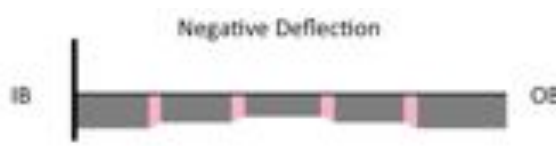

Mositive Deflection

FLAP2

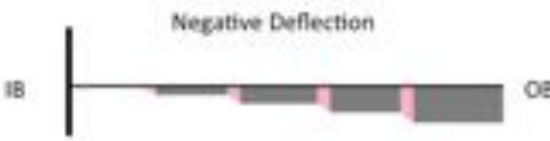

Positve Deflection

fuss
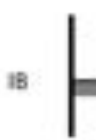

Negattve Deflection

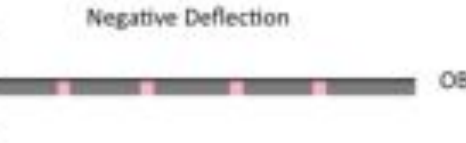

Posibve Deflection

FLAPI0

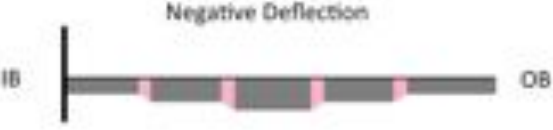

hositive Deflectian

FLAP3

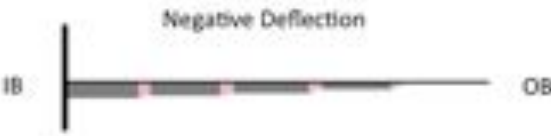

Positive Deflection

fLAP6

Negabve Defiection

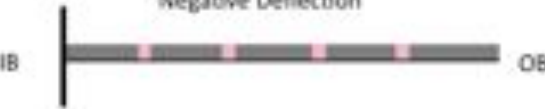

Positive Deflection

FLAP11

Fig. 15 - VCCTEF Flap Configurations (Courtesy of University of Washington Aeronautical Laboratory)

The test run matrix for the VCCTEF is shown in Table 2. 


\begin{tabular}{|l|c|c|c|c|c|c|c|c|c|c|c|c|c|}
\hline FLAP & 0 & 1 & 2 & 3 & 4 & 5 & 6 & 7 & 8 & 9 & 10 & 11 & 12 \\
\hline \hline$q_{\infty}=0-20 \mathrm{psf}, \alpha=0^{\circ}$ & 18 & 25 & 32 & & 42 & & 55 & & 73 & 79 & 86 & 93 & \\
\hline$q_{\infty}=0-20 \mathrm{psf}, \alpha=1^{\circ}$ & 19 & 26 & 33 & 38 & 43 & 49 & 56 & 64 & 74 & 80 & 87 & 94 & 88 \\
\hline$q_{\infty}=0-20 \mathrm{psf}, \alpha=1.5^{\circ}$ & & 27 & 34 & & & & & & & & & & \\
\hline$q_{\infty}=0-20 \mathrm{psf}, \alpha=2^{\circ}$ & 20 & 28 & 35 & 39 & 44 & 50 & 57 & 65 & 75 & 81 & 89 & 95 & \\
\hline$q_{\infty}=0-20 \mathrm{psf}, \alpha=2.5^{\circ}$ & & 29 & 36 & & & & & & & & & & \\
\hline$q_{\infty}=0-20 \mathrm{psf}, \alpha=3^{\circ}$ & & 30 & & 40 & 45 & 51 & 58 & 66 & 76 & 82 & 90 & 96 & \\
\hline$q_{\infty}=0-20 \mathrm{psf}, \alpha=4^{\mathrm{o}}$ & 22 & & & 41 & 46 & 52 & 59 & 67 & 77 & 83 & 91 & 97 & \\
\hline$q_{\infty}=0-20 \mathrm{psf}, \alpha=4.5^{\circ}$ & & & & & 47 & 53 & & & & & & & \\
\hline$q_{\infty}=0-20 \mathrm{psf}, \alpha=5^{\circ}$ & 23 & & & & & & 60,61 & 68 & 78 & 84 & 92 & 98 & \\
\hline$q_{\infty}=0-20 \mathrm{psf}, \alpha=5.5^{\circ}$ & & & & & & & 62 & & & & & & \\
\hline$q_{\infty}=0-20 \mathrm{psf}, \alpha=6^{\circ}$ & & & & & & & & & & & & 99 & \\
\hline$q_{\infty}=0-20 \mathrm{psf}, \alpha=6.5^{\circ}$ & & & & & & & & & & & & 100 & \\
\hline$q_{\infty}=10 \mathrm{psf}, \alpha=-2^{\circ}-10.5^{\circ}$ & 117 & & & & & & & 121 & & & & & \\
\hline$q_{\infty}=15 \mathrm{psf}, \alpha=-2^{\circ}-8.5^{\circ}$ & 118 & & & & & & & 122 & & & & & \\
\hline$q_{\infty}=20 \mathrm{psf}, \alpha=-2^{\mathrm{o}}-7^{\circ}$ & 104 & 106 & & & & & 111 & 109 & & & & 102 & 113 \\
\hline$q_{\infty}=25 \mathrm{psf}, \alpha=-2^{\circ}-5.5^{\circ}$ & 116 & 107 & & & & & & 123 & & & & & \\
\hline$q_{\infty}=30 \mathrm{psf}, \alpha=-2^{\circ}-4.5^{\circ}$ & 120 & & & & & & & 125 & & & & \\
\hline
\end{tabular}

Table 2 - Test UW2087 Run Matrix

The VCCTEF segment positions for these configurations are shown in Table 3. The following convention is used. A flap section is a portion of the VCCTEF along the spanwise direction. There are 5 flap sections numbered from 1 at the inboard to 5 at the outboard. Each flap section is comprised of three camber segments labeled as A for the innermost camber segment, $\mathrm{B}$ for the middle camber segment, and $\mathrm{C}$ for the trailing edge camber segment. This is shown in Fig. 16. The position angle is denoted by $(a / b / c)$ where $a, b$, and $c$ are flap positions in degrees relative to the forward flap segment. For segment $\mathrm{A}$, the position angle is with respect to the fixed wing portion.

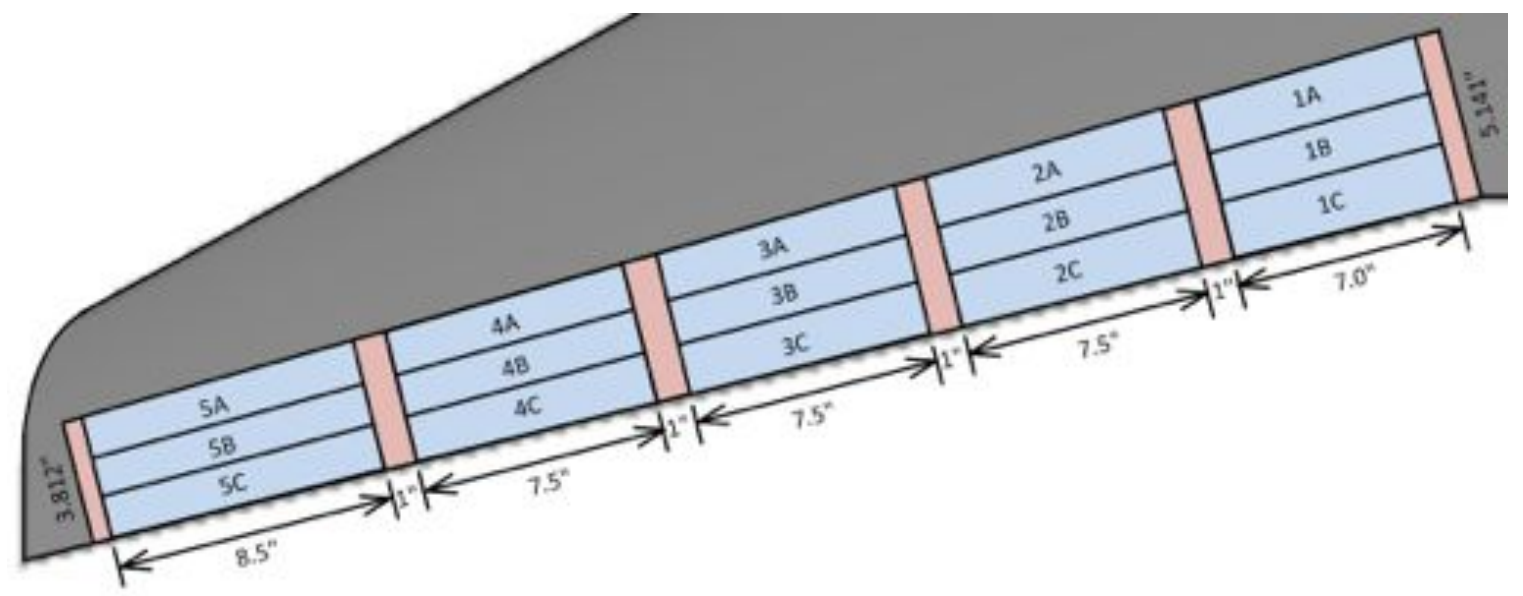

Fig. 16 - VCCTEF Flap Notation 


\begin{tabular}{|c|c|c|c|c|c|c|}
\hline FLAP & Run & Section 1 & Section 2 & Section 3 & Section 4 & Section 5 \\
\hline \hline 1 & 25 & $(5 / 4 / 9)$ & $(6 / 7 / 9)$ & $(9 / 7 / 10)$ & $(7 / 8 / 10)$ & $(6 / 9 / 9)$ \\
\hline $1^{*}$ & 106 & $(1 / 7 / 11)$ & $(3 / 9 / 7)$ & $(5 / 7 / 8)$ & $(5 / 9 / 7)$ & $(5 / 10 / 10)$ \\
\hline $1^{* *}$ & 107 & $(1 / 7 / 11)$ & $(3 / 9 / 7)$ & $(5 / 7 / 8)$ & $(5 / 9 / 7)$ & $(5 / 10 / 10)$ \\
\hline \multirow{2}{*}{2} & 32 & $(3 / 2 / 10)$ & $(4 / 5 / 4)$ & $(5 / 3 / 4)$ & $(4 / 6 / 5)$ & $(5 / 10 / 10)$ \\
& 36 & $(2 / 3 / 9)$ & $(4 / 5 / 4)$ & $(5 / 3 / 5)$ & $(4 / 6 / 4)$ & $(4 / 10 / 10)$ \\
\hline \multirow{2}{*}{3} & 38 & $(2 / 3 / 5)$ & $(5 / 6 / 5)$ & $(6 / 6 / 11)$ & $(5 / 6 / 5)$ & $(4 / 6 / 5)$ \\
& 41 & $(2 / 3 / 5)$ & $(4 / 6 / 5)$ & $(4 / 6 / 11)$ & $(4 / 6 / 5)$ & $(3 / 6 / 5)$ \\
\hline \multirow{2}{*}{4} & 42 & $(2 / 5 / 9)$ & $(3 / 6 / 3)$ & $(4 / 1 / 6)$ & $(2 / 5 / 2)$ & $(0 / 0 / 0)$ \\
& 47 & $(1 / 4 / 9)$ & $(3 / 6 / 2)$ & $(4 / 1 / 6)$ & $(2 / 5 / 1)$ & $(0 / 0 / 0)$ \\
\hline \multirow{3}{*}{5} & 49 & $(0 / 0 / 0)$ & $(2 / 5 / 1)$ & $(4 / 2 / 7)$ & $(3 / 5 / 6)$ & $(3 / 8 / 9)$ \\
& 51 & $(0 / 0 / 0)$ & $(2 / 5 / 1)$ & $(4 / 2 / 7)$ & $(3 / 5 / 6)$ & $(3 / 8 / 9)$ \\
& 53 & $(0 / 0 / 0)$ & $(2 / 5 / 1)$ & $(4 / 2 / 7)$ & $(3 / 5 / 5)$ & $(3 / 8 / 9)$ \\
\hline & 55 & $(1 / 1 / 1)$ & $(0 / 2 / 0)$ & $(0 / 0 / 2)$ & $(0 / 0 / 0.5)$ & $(0 / 0 / 0)$ \\
& 61 & $(1 / 1 / 1)$ & $(0 / 2 / 0)$ & $(0 / 0 / 2)$ & $(0 / 0 / 0.5)$ & $(0 / 0 / 0)$ \\
& 62 & $(1 / 1 / 1)$ & $(0 / 2 / 0)$ & $(0 / 0 / 2)$ & $(0 / 0 / 0.5)$ & $(0 / 0 / 0)$ \\
& 111 & $(1 / 1 / 1)$ & $(0 / 1 / 1)$ & $(0 / 0 / 2)$ & $(0 / 0 / 0)$ & $(0 / 0 / 0)$ \\
\hline 7 & 64 & $(3 / 3 / 4)$ & $(2 / 3 / 2)$ & $(0 / 0 / 3)$ & $(0 / 0 / 0)$ & $(0 / 0 /-2)$ \\
\hline $7 *$ & 109 & $(3 / 4 / 3)$ & $(2 / 5 / 1)$ & $(2 / 0 / 2)$ & $(0 /-1 / 0)$ & $(0 / 0 /-2)$ \\
\hline \multirow{2}{*}{8} & 73 & $(4 / 0 / 0)$ & $(4 / 0 / 0)$ & $(5 / 0 / 0)$ & $(5 / 0 / 0)$ & $(4 / 0 / 0)$ \\
& 78 & $(3 / 0 / 0)$ & $(4 / 0 / 0)$ & $(5 / 0 / 0)$ & $(4 / 0 / 0)$ & $(4 / 0 / 0)$ \\
\hline \multirow{3}{*}{9} & 79 & $(0 / 0 / 6)$ & $(0 / 0 / 6)$ & $(0 / 0 / 8)$ & $(0 / 0 / 6)$ & $(0 / 0 / 6)$ \\
& 81 & $(0 / 0 / 6)$ & $(0 / 0 / 6)$ & $(0 / 0 / 8)$ & $(0 / 0 / 6)$ & $(0 / 0 / 6)$ \\
& 84 & $(0 / 0 / 6)$ & $(0 / 0 / 6)$ & $(0 / 0 / 8)$ & $(0 / 0 / 6)$ & $(0 / 0 / 6)$ \\
\hline \multirow{2}{*}{10} & 86 & $(2 / 3 / 2)$ & $(3 / 3 / 1)$ & $(4 / 2 / 5)$ & $(2 / 5 / 2)$ & $(2 / 5 / 3)$ \\
& 91 & $(2 / 3 / 2)$ & $(3 / 3 / 1)$ & $(4 / 2 / 5)$ & $(2 / 5 / 2)$ & $(2 / 5 / 3)$ \\
& 92 & $(1 / 3 / 2)$ & $(3 / 3 / 1)$ & $(4 / 2 / 5)$ & $(2 / 5 / 2)$ & $(2 / 5 / 2)$ \\
\hline & 93 & $(-1 / 0 /-3)$ & $(0 / 0 /-6)$ & $(-1 /-4 / 0)$ & $(-2 / 0 /-5)$ & $(-4 / 0 /-4)$ \\
& 100 & $(-1 / 0 /-4)$ & $(0 / 0 /-5)$ & $(-1 /-3 / 0)$ & $(-2 / 0 /-5)$ & $(-3 / 0 /-4)$ \\
\hline
\end{tabular}

Table 3 - VCCTEF Deflections

\section{Aeroelastic Effects on Lift and Drag}

The aeroelastic deflections of a flexible swept back wind tunnel model contribute significantly to the aerodynamic performance due to the wash-out effect resulting from wing bending and twist. Additionally, the VCCTEF deflections also contribute to angle of attack changes. The aeroelastic angle of attack of a wing section can be expressed as ${ }^{6}$

$$
\alpha_{c}(y)=\alpha-\alpha_{i}(y)-\gamma(\bar{y}) \cos \Lambda-\Theta(\bar{y}) \cos \Lambda-\frac{d W(\bar{y})}{d \bar{y}} \sin \Lambda+\sum_{i=1}^{N} \frac{\partial \alpha_{c}}{\partial \delta_{i}} \delta_{i}\left(y_{h}\right) \cos \Lambda_{h}
$$

where $\alpha$ is the geometric angle of attack of the wing section about the pitch axis $y, \alpha_{i}$ is the induced angle of attack due to the downwash about the pitch axis $y, \gamma$ is the wing pre-twist angle about the elastic axis $\bar{y}=y / \cos \Lambda$ (positive nose down), $\Theta$ is the wing torsional twist about the elastic axis $\bar{y}$ (positive nose down), $W$ is wing vertical bending along the elastic axis (positive upward), $\Lambda$ is the sweep angle of the elastic axis, $\delta_{i}$ is the absolute deflection of the $i$-th flap segment of the VCCTEF about the hinge axis $y_{h}$ which has a sweep angle of $\Lambda_{h}$, and $\partial \alpha_{c} / \partial \delta_{i}$ is the angle of attack sensitivity or camber control derivative due to the VCCTEF flap deflection.

The following convention is used. A quantity expressed with the independent variable $y$ is one with respect to the streamwise direction, e.g., $c(y)$ is the sectional chord in the streamwise direction. A quantity expressed with the independent variable $\bar{y}$ is one with respect to the elastic axis; e.g., $\Theta(\bar{y})$ is the wing twist about the elastic axis. The quantity $\delta_{i}\left(y_{h}\right)$ is the flap deflection about the hinge axis.

The camber control derivative $\frac{\partial \alpha_{c}}{\partial \delta_{i}}$ can be directly estimated from thin airfoil potential flow theory by evaluating the follow- 
ing integral transform with the potential kernel function $f(\theta)=\cos \theta-1$ as $^{7}$

$$
\frac{\partial \alpha}{\partial \delta_{i}}=-\frac{1}{\pi} \int_{\theta_{i}}^{\theta_{i+1}} f(\theta) d \theta=-\frac{1}{\pi} \int_{\theta_{i}}^{\theta_{i+1}}(\cos \theta-1) d \theta
$$

where

$$
\begin{aligned}
x^{\prime} & =\frac{c^{\prime}}{2}(1-\cos \theta) \\
c^{\prime}-x_{i}^{\prime} & =\frac{c^{\prime}}{2}\left(1-\cos \theta_{i}\right)
\end{aligned}
$$

and $c^{\prime}$ is the airfoil chord and $x_{i}^{\prime}$ is the flap hinge position of the $i$-th flap segment measured normal to the hinge axis from the trailing edge and is given by

$$
x_{i}^{\prime}=(n+1-i) c_{f}^{\prime}
$$

where $c_{f}^{\prime}$ is the flap chord of a camber segment measured normal to the hinge axis.

So the first hinge position is at $x_{1}^{\prime}=N c_{f}^{\prime}$, the last hinge position is at $x_{N}^{\prime}=c_{f}^{\prime}$, and the trailing edge position is at $x_{N+1}^{\prime}=0$.

This integral is evaluated as

$$
\frac{\partial \alpha_{c}}{\partial \delta_{i}}=\left.\frac{\cos ^{-1}\left(-c^{*}\right)-\sqrt{1-c^{* 2}}}{\pi}\right|_{c_{i}^{*}} ^{c_{i+1}^{*}}
$$

where

$$
c^{*}=1-2 \frac{x_{i}^{\prime}}{c^{\prime}}
$$

The sectional lift coefficient is expressed as

$$
c_{L}(y)=c_{L_{0}}(y)+c_{L_{\alpha}}(y)\left[\alpha(y)-\gamma(\bar{y}) \cos \Lambda-\Theta(\bar{y}) \cos \Lambda-\frac{d W(\bar{y})}{d \bar{y}} \sin \Lambda+\sum_{i=1}^{N} \frac{\partial \alpha_{c}}{\partial \delta_{i}} \delta_{i}\left(y_{h}\right) \cos \Lambda_{h}\right]
$$

where $c_{L_{0}}$ is the sectional lift coefficient at zero angle of attack and $c_{L_{\alpha}}$ is the sectional lift curve slope, both of which account for the induced angle of attack.

The sectional lift coefficient parameters $c_{L_{0}}$ and $c_{L_{\alpha}}$ vary along the wing span and can be estimated from a three-dimensional aerodynamic analysis using a panel code or a CFD (Computational Fluid Dynamics) code.

Using aerodynamic strip theory, the total wing lift coefficient can be computed by integrating the local lift coefficient as follows:

$$
C_{L}=\frac{1}{S} \int_{-b / 2}^{b / 2} c_{L}(y) c(y) d y
$$

where $c$ is the local chord length in the streamwise direction, and $S$ is the wing reference area.

The lift coefficient can also be expressed in the standard form

$$
C_{L}=C_{L_{0}}+C_{L_{\alpha}}^{*} \alpha+C_{L_{\delta}}^{*} \delta+\Delta C_{L_{e}}
$$

where $C_{L_{0}}^{*}$ is the rigid-wing lift coefficient at zero angle of attack, $C_{L_{\alpha}}^{*}$ is the rigid-wing lift curve slope of finite-aspect ratio wing, $C_{L_{\delta}}^{*}$ is the vector of rigid-wing lift coefficient sensitivities due to the VCCTEF deflection, $\delta=\left[\begin{array}{lll}\delta_{1} & \ldots & \delta_{N}\end{array}\right]^{\top}$ is the VCCTEF deflection vector, and $\Delta C_{L_{e}}$ is the incremental lift coefficients due to wing flexibility.

These quantities may be evaluated as

$$
\begin{aligned}
C_{L_{0}}^{*} & =\frac{1}{S} \int_{-b / 2}^{b / 2}\left[c_{L_{0}}(y)-c_{L_{\alpha}} \gamma(y) \cos \Lambda\right] c(y) d y \\
C_{L_{\alpha}}^{*} & =\frac{1}{S} \int_{-b / 2}^{b / 2} c_{L_{\alpha}}(y) c(y) d y \\
C_{L_{\delta}}^{*} & =\frac{1}{S} \int_{-b / 2}^{b / 2} c_{L_{\alpha}}(y) \frac{\partial \alpha_{c}}{\partial \delta} \cos \Lambda_{h} c(y) d y \\
\Delta C_{L_{e}} & =\frac{1}{S} \int_{-b / 2}^{b / 2} c_{L_{\alpha}}(y)\left[-\Theta(\bar{y}) \cos \Lambda-\frac{d W(\bar{y})}{d \bar{y}} \sin \Lambda\right] c(y) d y
\end{aligned}
$$


where $\frac{\partial \alpha_{c}}{\partial \delta}=\left[\begin{array}{lll}\frac{\partial \alpha_{c}}{\partial \delta_{1}} & \ldots & \frac{\partial \alpha_{c}}{\partial \delta_{N}}\end{array}\right]$

The aeroelastic deflections of a wing can be modeled by the following equations:

$$
\begin{aligned}
\frac{\partial}{\partial \bar{y}}\left\{\left[G J+E B_{1}\left(\gamma^{\prime}\right)^{2}\right] \frac{\partial \Theta}{\partial \bar{y}}-E B_{2} \gamma^{\prime} \frac{\partial^{2} W}{\partial \bar{y}^{2}}\right\} & =\left[\left(c_{m_{a c}}+c_{m_{\delta}} \delta\right) c+\frac{e}{\cos \Lambda} c_{L}\right] q_{\infty} \cos ^{2} \Lambda c \\
\frac{\partial^{2}}{\partial \bar{y}^{2}}\left(E I \frac{\partial^{2} W}{\partial \bar{y}^{2}}-E B_{2} \gamma^{\prime} \frac{\partial \Theta}{\partial \bar{y}}\right) & =c_{L} q_{\infty} \cos \Lambda c
\end{aligned}
$$

where $e$ is the aerodynamic center offset measured perpendicular to the elastic axis, $c_{m_{a c}}$ is the sectional pitching moment coefficient about the aerodynamic center defined in the streamwise direction, $c_{m_{\delta}}$ is the vector of pitching moment coefficient sensitivities about the aerodynamic center defined in the streamwise direction for the VCCTEF, and $B_{1}$ and $B_{2}$ are the bendingtorsion coupling constants which are generally small for slightly twisted wings and therefore are usually neglected.

Using thin airfoil potential flow theory, the pitching moment coefficient sensitivity about the aerodynamic center, which is taken to be at the quarter-chord location, of the $i$-th flap segment can be estimated using a kernel function $g(\theta)=\cos 2 \theta-\cos \theta$ as

$$
c_{m_{\delta_{i}}}=-\frac{c_{L_{\alpha}}}{4 \pi} \int_{\theta_{i}}^{\theta_{i+1}}(\cos 2 \theta-\cos \theta) d \theta
$$

where $c_{L_{\alpha}}$ is the sectional lift curve slope from Eq. (8).

This results in a theoretical expression as

$$
c_{m_{\delta_{i}}}=\left.\frac{c_{L_{\alpha}}\left(1+c^{*}\right) \sqrt{1-c^{* 2}}}{4 \pi}\right|_{c_{i}^{*}} ^{c_{i+1}^{*}}
$$

The Kutta-Joukowski theorem states that, for a two-dimensional airfoil, the lift force per unit length is $L=\rho_{\infty} V_{\infty} \Gamma$, where $\Gamma$ is the circulation. ${ }^{7}$ Then, from Eq. (8), the lift circulation is defined as

$$
\begin{aligned}
\Gamma(y) & =\frac{1}{2} V_{\infty} c_{L}(y) c(y) \\
& =\frac{1}{2} V_{\infty}\left\{c_{L_{0}}(y)+c_{L_{\alpha}}(y)\left[\alpha-\gamma(\bar{y}) \cos \Lambda-\Theta(\bar{y}) \cos \Lambda-\frac{d W(\bar{y})}{d \bar{y}} \sin \Lambda+\sum_{i=1}^{N} \frac{\partial \alpha_{c}}{\partial \delta_{i}} \delta_{i}\left(y_{h}\right) \cos \Lambda_{h}\right]\right\} c(y)
\end{aligned}
$$

The lift circulation shows that the aeroelastic deflections can influence the spanwise lift distribution, hence induced drag. As a wing becomes more flexible, aeroelastic deflections at off-design cruise conditions can cause induced drag penalty. By controlling the aeroelastic deflections using the VCCTEF, the spanwise lift distribution can be tailored for off-design aerodynamic performance. It should be noted that the VCCTEF can provide the same potential benefit for a stiff and, in the limit, even rigid wing design since the VCCTEF can directly alter the spanwise lift distribution via camber control. To achieve optimal off-design aerodynamic performance, a multi-disciplinary optimization can be conducted to determine the optimal VCCTEF deflections at functions of flight conditions inside a flight envelope. As a matter of fact, the very terminology of "design" and "off-design" flight conditions may need to be revised, since with the VCCTEF it might be possible to approach optimal lift-to-drag ratios at many flight conditions.

For a flexible swept back wing, the bending slope $\frac{d W}{d \bar{y}}$ which is normally positive causes a local wing wash-out twist which decreases the sectional angle of attack. As a result, the lift coefficient generally decreases at a given aircraft angle of attack when compared to the lift coefficient for a rigid wing. Therefore, to achieve the same lift coefficient, an increase in the angle of attack is required to compensate for the aeroelastic effect.

To model the aeroelastic effects, various modeling approaches are used. A standard approach is to use a finite-element model coupled with an aerodynamic solver such as a panel code or a CFD code. To use the finite-element method, the structural properties of a wing need to be established accurately. This information is then used to predict the aeroelastic deflection. Even though such a method is considered to be standard, the modeling accuracy can still be subject to high uncertainty. Stiffness uncertainty as well as aerodynamic prediction uncertainty can result in inaccurate results.

One approach to reducing modeling uncertainty in the data analysis using strictly experimental data is Galerkin's method. Galerkin's method uses a series approximation of mode shapes using the analytical mode shapes of a simpler structure with the same boundary conditions. For a cantilever wing, the mode shapes can be approximated by a series of analytical mode shapes of a uniform cantilever beam. ${ }^{8}$ Since no structural information about the stiffness is required and the aeroelastic deflection measurements are available, Galerkin's method is well suited for use in the data analysis. The resulting formulation can add insight into the various physical effects involved in the wind tunnel test. A standard finite-element aerodynamic panel solution is presented in the paper by Precup, Mor, and Livne. ${ }^{9}$ In addition, a preliminary static aeroelastic analysis was also conducted 
by Rodriguez, Aftosmis, Nemec, and Smith using Cart3D inviscid CFD code coupled with a structural beam model. ${ }^{10}$ A finite-element modeling method coupled to a vortex-lattice aerodynamic solver is also being developed. All these modeling approaches are complementary to the work in the current paper.

An approximate solution of Eqs. (15) and (16) may be obtained using Galerkin's method by assuming the following solution:

$$
\begin{aligned}
\Theta(\bar{y}) & =\Psi(\bar{y}) \theta \\
W(\bar{y}) & =\Phi(\bar{y}) w
\end{aligned}
$$

where $\theta=\left[\begin{array}{lll}\theta_{1} & \ldots & \theta_{n}\end{array}\right]^{\top}$ and $w=\left[\begin{array}{lll}w_{1} & \ldots & w_{n}\end{array}\right]^{\top}$ are vectors of generalized coordinates, and $\Psi(\bar{y})=\left[\begin{array}{lll}\psi_{1}(\bar{y}) & \ldots & \psi_{n}(\bar{y})\end{array}\right]$ and $\Phi(\bar{y})=\left[\begin{array}{lll}\phi_{1}(\bar{y}) & \ldots & \phi_{n}(\bar{y})\end{array}\right]$ are shape functions selected to be the natural mode shapes of a uniform cantilever beam which are given by

$$
\begin{aligned}
& \psi_{i}(\bar{y})=\sqrt{2} \sin \frac{(2 i-1) \pi \bar{y}}{2 L} \\
& \phi_{i}(\bar{y})=\cosh \left(\beta_{i} \bar{y}\right)-\cos \left(\beta_{i} \bar{y}\right)-\frac{\cosh \left(\beta_{i} L\right)+\cos \left(\beta_{i} L\right)}{\sinh \left(\beta_{i} L\right)+\sin \left(\beta_{i} L\right)}\left[\sinh \left(\beta_{i} \bar{y}\right)-\sin \left(\beta_{i} \bar{y}\right)\right]
\end{aligned}
$$

where $\beta_{i} L$ are the eigenvalues corresponding to their natural mode shapes of beam bending.

Following the Galerkin's method, the torsion and bending equations are pre-multiplied by the torsion and bending shape functions $\Psi^{\top}(\bar{y})$ and $\Phi^{\top}(\bar{y})$, respectively, and then integrating over the wing span. This leads to a discretized matrix equation in terms of the generalized stiffnesses and generalized forces as

$$
\left[\begin{array}{cc}
K_{s_{\theta \theta}}+K_{a_{\theta \theta}} & K_{a_{\theta w}} \\
K_{a_{w \theta}} & K_{s_{w w}}+K_{a_{w w}}
\end{array}\right]\left[\begin{array}{c}
\theta \\
w
\end{array}\right]=\left[\begin{array}{c}
F_{\theta_{0}} \\
F_{w_{0}}
\end{array}\right]+\left[\begin{array}{c}
F_{\theta_{\alpha}} \\
F_{w_{\alpha}}
\end{array}\right] \alpha+\left[\begin{array}{c}
F_{\theta_{\delta}} \\
F_{w_{0}}
\end{array}\right] \delta
$$

where

$$
\begin{aligned}
& K_{s_{\theta \theta}}=\int_{0}^{L} G J \frac{d \Psi^{\top}(\bar{y})}{d \bar{y}} \frac{d \Psi(\bar{y})}{d \bar{y}} d \bar{y} \\
& K_{s_{w w}}=\int_{0}^{L} E I \frac{d^{2} \Phi^{\top}(\bar{y})}{d \bar{y}^{2}} \frac{d^{2} \Phi(\bar{y})}{d \bar{y}^{2}} d \bar{y} \\
& K_{a_{\theta \theta}}=-\int_{0}^{L} e(\bar{y}) c_{L_{\alpha}} \Psi^{\top}(\bar{y}) \Psi(\bar{y}) q_{\infty} \cos ^{2} \Lambda c(y) d \bar{y} \\
& K_{a_{\theta w}}=-\int_{0}^{L} e(\bar{y}) c_{L_{\alpha}} \Psi^{\top}(\bar{y}) \frac{d \Phi(\bar{y})}{d \bar{y}} q_{\infty} \sin \Lambda \cos \Lambda c(y) d \bar{y} \\
& K_{a_{w w}}=\int_{0}^{L} c_{L_{\alpha}} \Phi^{\top}(\bar{y}) \frac{d \Phi(\bar{y})}{d \bar{y}} q_{\infty} \sin \Lambda \cos \Lambda c(y) d \bar{y} \\
& K_{a_{w \theta}}=\int_{0}^{L} c_{L_{\alpha}} \Phi^{\top}(\bar{y}) \Psi(\bar{y}) q_{\infty} \cos ^{2} \Lambda c(y) d \bar{y} \\
& F_{\theta_{0}}=-\int_{0}^{L}\left\{c_{m_{a c}} c(y)+e(\bar{y})\left[\frac{c_{L_{0}}}{\cos \Lambda}-c_{L_{\alpha}} \gamma(\bar{y})\right]\right\} \Psi^{\top}(\bar{y}) q_{\infty} \cos ^{2} \Lambda c(y) d \bar{y} \\
& F_{\theta_{\alpha}}=-\int_{0}^{L} e(\bar{y}) c_{L_{\alpha}} \Psi^{\top}(\bar{y}) q_{\infty} \cos \Lambda c(y) d \bar{y} \\
& F_{\theta_{\delta}}=-\int_{0}^{L}\left[c_{m_{\delta}} c(y)+\frac{e(\bar{y}) c_{L_{\alpha}}}{\cos \Lambda} \frac{\partial \alpha_{c}}{\partial \delta} \cos \Lambda_{h}\right] \Psi^{\top} q_{\infty} \cos ^{2} \Lambda c(y) d \bar{y} \\
& F_{w_{0}}=\int_{0}^{L}\left[\frac{c_{L_{0}}}{\cos \Lambda}-c_{L_{\alpha}} \gamma(\bar{y})\right] \Phi^{\top}(\bar{y}) q_{\infty} \cos ^{2} \Lambda c(y) d \bar{y} \\
& F_{w_{\alpha}}=\int_{0}^{L} c_{L_{\alpha}} \Phi^{\top}(\bar{y}) q_{\infty} \cos \Lambda c(y) d \bar{y} \\
& F_{w_{\delta}}=\int_{0}^{L} c_{L_{\alpha}} \frac{\partial \alpha_{c}}{\partial \delta} \cos \Lambda_{h} \Phi^{\top}(\bar{y}) q_{\infty} \cos \Lambda c(y) d \bar{y}
\end{aligned}
$$


Note that these integrals are evaluated along the elastic axis $\bar{y} \in[0, L]$, where $L=\frac{b}{2 \cos \Lambda}$.

The solutions of the generalized coordinates are expressed as

$$
\begin{aligned}
{\left[\begin{array}{c}
\theta \\
w
\end{array}\right] } & =\left[\begin{array}{cc}
K_{s_{\theta \theta}}+K_{a_{\theta \theta}} & K_{a_{\theta w}} \\
K_{a_{w \theta}} & K_{s_{w w}}+K_{a_{w w}}
\end{array}\right]^{-1}\left\{\left[\begin{array}{l}
F_{\theta_{0}} \\
F_{w_{0}}
\end{array}\right]+\left[\begin{array}{c}
F_{\theta_{\alpha}} \\
F_{w_{\alpha}}
\end{array}\right] \alpha+\left[\begin{array}{c}
F_{\theta_{\delta}} \\
F_{w_{0}}
\end{array}\right] \delta\right\} \\
& =\left[\begin{array}{c}
\theta_{0} \\
w_{0}
\end{array}\right]+\frac{\partial}{\partial \alpha}\left[\begin{array}{c}
\theta \\
w
\end{array}\right] \alpha+q_{\infty} \frac{\partial}{\partial \delta}\left[\begin{array}{c}
\theta \\
w
\end{array}\right] \delta
\end{aligned}
$$

Thus, the aeroelastic deflections are obtained as functions of the angle of attack and the flap deflections as

$$
\begin{aligned}
& \Theta(\bar{y})=\Psi(\bar{y})\left(\theta_{0}+\frac{\partial \theta}{\partial \alpha} \alpha+\frac{\partial \theta}{\partial \delta} \delta\right) \\
& W(\bar{y})=\Phi(\bar{y})\left(w_{0}+\frac{\partial w}{\partial \alpha} \alpha+\frac{\partial w}{\partial \delta} \delta\right)
\end{aligned}
$$

Note that $K_{a_{\theta \theta}}, K_{a_{\theta w}}, K_{a_{w \theta}}$, and $K_{a_{w w}}$ are dependent on $q_{\infty}$. The stiffness matrix inverse can be expressed as

$$
\left(K_{s}+q_{\infty} \frac{K_{a}}{q_{\infty}}\right)^{-1}=K_{s}^{-1}-q_{\infty} K_{s}^{-1} \frac{K_{a}}{q_{\infty}} K_{s}^{-1}\left(I+q_{\infty} \frac{K_{a}}{q_{\infty}} K_{s}^{-1}\right)^{-1}
$$

where $\frac{K_{a}}{q_{\infty}}$ is the aerodynamic generalized influence coefficients matrix which is independent of $q_{\infty}$.

To derive an explicit dependency of the lift coefficient on the dynamic pressure for the purpose of analyzing the wind tunnel test data, consider the case when $q_{\infty} \frac{K_{a}}{q_{\infty}} K_{s}^{-1} \ll I$ which is generally applicable for a low-speed wind tunnel test at a dynamic pressure well below the divergence dynamic pressure, then the stiffness matrix inverse can be approximated as

$$
\left(K_{s}+q_{\infty} \frac{K_{a}}{q_{\infty}}\right)^{-1} \approx K_{s}^{-1}-q_{\infty} K_{s}^{-1} \frac{K_{a}}{q_{\infty}} K_{s}^{-1}+q_{\infty}^{2} K_{s}^{-1} \frac{K_{a}}{q_{\infty}} K_{s}^{-1} \frac{K_{a}}{q_{\infty}} K_{s}^{-1}
$$

Since all the generalized force terms are dependent on $q_{\infty}$, therefore one can express

$$
\begin{aligned}
{\left[\begin{array}{c}
\theta_{0} \\
w_{0}
\end{array}\right] } & =\left[\begin{array}{l}
A_{0_{\theta}} \\
A_{0_{w}}
\end{array}\right] q_{\infty}+\left[\begin{array}{c}
B_{0_{\theta}} \\
B_{0_{w}}
\end{array}\right] q_{\infty}^{2}+\left[\begin{array}{l}
C_{0_{\theta}} \\
C_{0_{w}}
\end{array}\right] q_{\infty}^{3} \\
\frac{\partial}{\partial \alpha}\left[\begin{array}{c}
\theta \\
w
\end{array}\right] & =\left[\begin{array}{l}
A_{\alpha_{\theta}} \\
A_{\alpha_{w}}
\end{array}\right] q_{\infty}+\left[\begin{array}{c}
B_{\alpha_{\theta}} \\
B_{\alpha_{w}}
\end{array}\right] q_{\infty}^{2}+\left[\begin{array}{c}
C_{\alpha_{\theta}} \\
C_{\alpha_{w}}
\end{array}\right] q_{\infty}^{3} \\
\frac{\partial}{\partial \delta}\left[\begin{array}{c}
\theta \\
w
\end{array}\right] & =\left[\begin{array}{l}
A_{\delta_{\theta}} \\
A_{\delta_{w}}
\end{array}\right] q_{\infty}+\left[\begin{array}{l}
B_{\delta_{\theta}} \\
B_{\delta_{w}}
\end{array}\right] q_{\infty}^{2}+\left[\begin{array}{c}
C_{\delta_{\theta}} \\
C_{\delta_{w}}
\end{array}\right] q_{\infty}^{3}
\end{aligned}
$$

Since the aeroelastic deflections are dependent on $\alpha$ and $\delta$ as well as $q_{\infty}$, the effective lift coefficient parameters are computed as

$$
\begin{aligned}
C_{L_{0}} & =C_{L_{0}}^{*}+a_{0} q_{\infty}+b_{0} q_{\infty}^{2}+c_{0} q_{\infty}^{3} \\
C_{L_{\alpha}} & =C_{L_{\alpha}}^{*}+a_{\alpha} q_{\infty}+b_{\alpha} q_{\infty}^{2}+c_{\alpha} q_{\infty}^{3} \\
C_{L_{\delta}} & =C_{L_{\delta}}^{*}+a_{\delta} q_{\infty}+b_{\delta} q_{\infty}^{2}+c_{\delta} q_{\infty}^{3}
\end{aligned}
$$

where

$$
\begin{aligned}
& a_{(0, \alpha, \delta)}=\frac{1}{S} \int_{-b / 2}^{b / 2} c_{L_{\alpha}}(y)\left[-\Psi(\bar{y}) A_{(0, \alpha, \delta)_{\theta}} \cos \Lambda-\frac{d \Phi(\bar{y})}{d \bar{y}} A_{(0, \alpha, \delta)_{w}} \sin \Lambda\right] c(y) d y \\
& b_{(0, \alpha, \delta)}=\frac{1}{S} \int_{-b / 2}^{b / 2} c_{L_{\alpha}}(y)\left[-\Psi(\bar{y}) B_{(0, \alpha, \delta)_{\theta}} \cos \Lambda-\frac{d \Phi(\bar{y})}{d \bar{y}} B_{(0, \alpha, \delta)_{w}} \sin \Lambda\right] c(y) d y \\
& c_{(0, \alpha, \delta)}=\frac{1}{S} \int_{-b / 2}^{b / 2} c_{L_{\alpha}}(y)\left[-\Psi(\bar{y}) C_{(0, \alpha, \delta)_{\theta}} \cos \Lambda-\frac{d \Phi(\bar{y})}{d \bar{y}} C_{(0, \alpha, \delta)_{w}} \sin \Lambda\right] c(y) d y
\end{aligned}
$$

Note that these integral are evaluated in the wing pitch axis $y \in\left[0, \frac{b}{2}\right]$. 
Thus, the lift coefficient of a flexible wing is expressed as

$$
C_{L}=C_{L}^{*}+\left(a_{0}+a_{\alpha} \alpha+a_{\delta} \delta\right) q_{\infty}+\left(b_{0}+b_{\alpha} \alpha+b_{\delta} \delta\right) q_{\infty}^{2}+\left(c_{0}+c_{\alpha} \alpha+c_{\delta} \delta\right) q_{\infty}^{3}
$$

where $C_{L}^{*}$ is the rigid-wing lift coefficient and the remaining terms are due to the effect of aeroelasticity.

Equation (51) can be used for analyzing the wind tunnel test data, as will be shown in the next section. Note that if a swept back wing experiences a nose-down twist $(\Psi(\bar{y})>0)$ and upward bending $\left(\frac{d \Phi(\bar{y})}{d \bar{y}}>0\right)$, then the terms $a_{(0, \alpha, \delta)}$ are negative since they are due to the positive terms $A_{(0, \alpha, \delta)_{\theta}}$ and $A_{(0, \alpha, \delta)_{w}}$ which come from $K_{s}^{-1}$ which is always positive.

Note that when $q_{\infty}=0, C_{L}=C_{L}^{*}$. This suggests that one can estimate the rigid-wing lift coefficient by analyzing the lift coefficient variation with the dynamic pressure. However, this will require data extrapolation since aerodynamic measurements at low dynamic pressure are generally not reliable.

If the dynamic pressure is held constant and if aeroelastic deflection measurements are available, then this information can be used to estimate the rigid-wing lift coefficient. In this case, the effective lift coefficient parameters are expressed as

$$
\begin{aligned}
& C_{L_{0}}=C_{L_{0}}^{*}-\frac{1}{S} \int_{-b / 2}^{b / 2} c_{L_{\alpha}}(y)\left[\Theta_{0}(\bar{y}) \cos \Lambda+\frac{d W_{0}(\bar{y})}{d \bar{y}} \sin \Lambda\right] c(y) d y \\
& C_{L_{\alpha}}=C_{L_{\alpha}}^{*}-\frac{1}{S} \int_{-b / 2}^{b / 2} c_{L_{\alpha}}(y)\left[\frac{\partial \Theta(\bar{y})}{\partial \alpha} \cos \Lambda+\frac{\partial}{\partial \alpha}\left(\frac{d W(\bar{y})}{d \bar{y}}\right) \sin \Lambda\right] c(y) d y \\
& C_{L_{\delta}}=C_{L_{\delta}}^{*}-\frac{1}{S} \int_{-b / 2}^{b / 2} c_{L_{\alpha}}(y)\left[\frac{\partial \Theta(\bar{y})}{\partial \delta} \cos \Lambda+\frac{\partial}{\partial \delta}\left(\frac{d W(\bar{y})}{d \bar{y}}\right) \sin \Lambda\right] c(y) d y
\end{aligned}
$$

Since $\Theta$ and $\frac{d W}{d \bar{y}}$ are generally positive for a wing with positive lift, the effect of aeroelasticity on a swept back wing is to reduce lift and lift coefficient sensitivity due to a flap deflection which gives rise to control reversal.

Going back to the aeroelastic angle of attack expression, one can write

$$
\begin{aligned}
\alpha_{c}(y) & =\alpha\left[1-\frac{\partial \Theta(\bar{y})}{\partial \alpha} \cos \Lambda-\frac{\partial}{\partial \alpha}\left(\frac{d W(\bar{y})}{d \bar{y}}\right) \sin \Lambda\right]-\alpha_{i}(y)-\gamma(\bar{y}) \cos \Lambda-\Theta_{0}(\bar{y}) \cos \Lambda-\frac{d W_{0}(\bar{y})}{d \bar{y}} \sin \Lambda \\
& +\left[\frac{\partial \alpha_{c}}{\partial \delta} \cos \Lambda_{h}-\frac{\partial \Theta(\bar{y})}{\partial \delta} \cos \Lambda-\frac{\partial}{\partial \delta}\left(\frac{d W(\bar{y})}{d \bar{y}}\right) \sin \Lambda\right] \delta\left(y_{h}\right)
\end{aligned}
$$

It can be seen that the aeroelastic deflections can cause the desired sectional angle of attack to be non-optimal. The effect of the adaptive aeroelastic wing shaping control by the VCCTEF is captured in the last term. The term $\frac{\partial \alpha_{c}}{\partial \delta}$ is the rigid camber control to compensate for the non-optimal sectional angle of attack. The two terms $\frac{\partial \Theta}{\partial \delta}$ and $\frac{\partial}{\partial \delta}\left(\frac{d W}{d \bar{y}}\right)$ are the aeroelastic wing shaping control by leveraging wing flexibility to change the wash-out twist of a wing in order to achieve improved aerodynamic performance. Thus, the effect of adaptive aeroelastic wing shaping control is to optimize the span load at any operating point inside a given flight envelope using both the camber control and aeroelastic wing shaping control.

\section{Wind Tunnel Experimental Data}

There was a total of 125 useful test runs. Test data consist of force, moment, and aeroelastic deflection measurements, and were collected during $\alpha$-sweep and $q_{\infty}$-sweep runs. For clarity, $\alpha$ is the angle of attack of the flexible wing wind tunnel model, which is defined as the angle between incoming air and the root attachment of the wing. The force and moment measurements were provided by the external balance. The aeroelastic deflection measurements were provided by the VICON motion tracking system.

The lift curve and drag polar for the baseline FLAP0 configuration are plotted in Figs. 17 and 18. The data come from 20 different runs. As can be seen, the data show high degree of repeatability and correlation. The coefficient of correlation for the linear regression of the lift curve is 0.9999. Figures 19 to 48 are the lift curves and drag polars for all the VCCTEF configurations as compared to the baseline FLAP0 configurations. These data are taken from $q_{\infty}$-sweep runs at constant angles of attack. A data filter was conducted to retain only data at $q_{\infty}=20 \mathrm{psf}$. Figures 41 to 48 are the lift curves and drag polars for FLAP1, FLAP7, and FLAP12 configurations taken from $\alpha$-sweep runs 102 to 113 at $q_{\infty}=20$ psf. The reason they are plotted separately is that the VCCTEF deflections for FLAP1 and FLAP7 configurations for these runs were observed to be not the same deflections as those for $q_{\infty}$-sweep runs 18 to 100, as shown in Table 3. Thus, these configurations are denoted FLAP1*, FLAP1**, and FLAP7*. 
FLAP0, $\mathrm{q}_{\alpha}=20 \mathrm{psf}, \mathrm{C}_{\mathrm{L}}=0.1778+4.1482 \alpha, \mathrm{R}=0.9999$

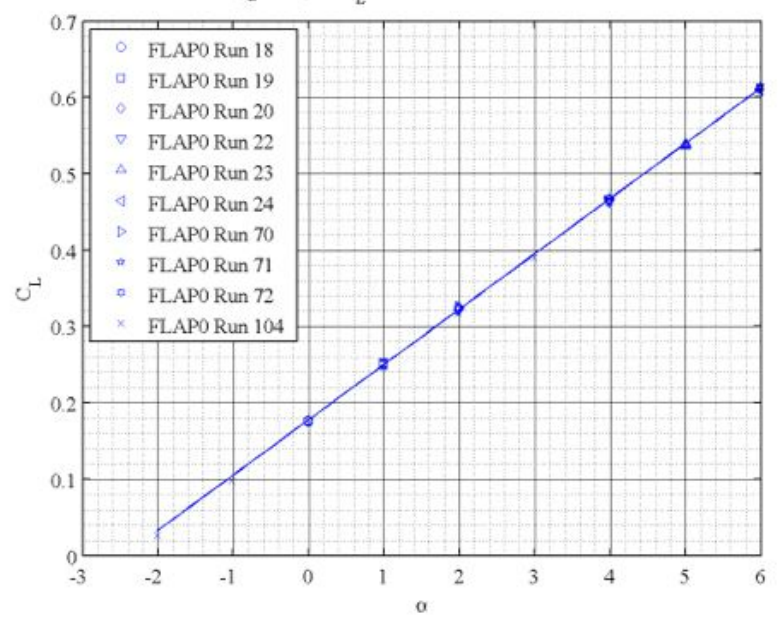

Fig. 17 - FLAP0 $C_{L}$ vs. $\alpha$

FLAP1 vs. FLAP0, $q_{\alpha}=20$ psf, $C_{L}=0.3669+3.6708 \alpha, R=0.9990$

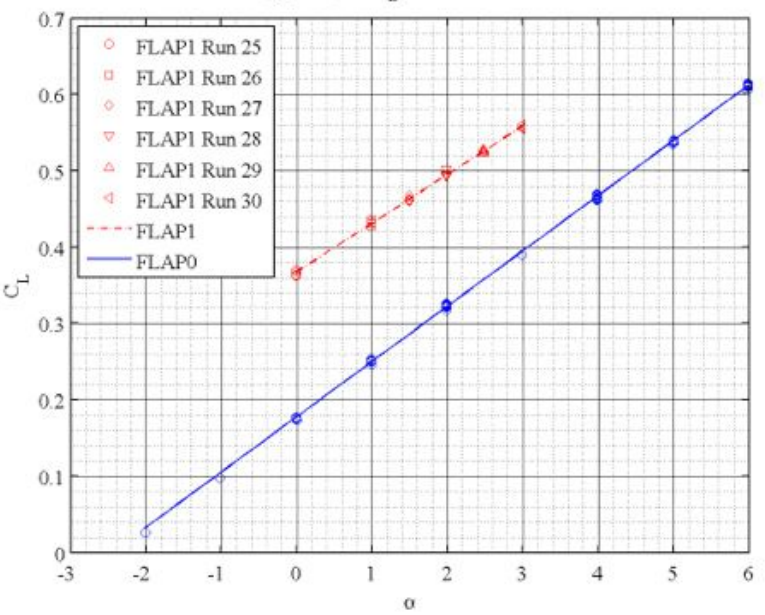

Fig. 19 - FLAP1 $C_{L}$ vs. $\alpha$

FLAP2 vs. FLAP0, $\mathrm{q}_{\alpha}=20$ psf, $\mathrm{C}_{\mathrm{L}}=0.2994+3.8414 \alpha, \mathrm{R}=0.9996$

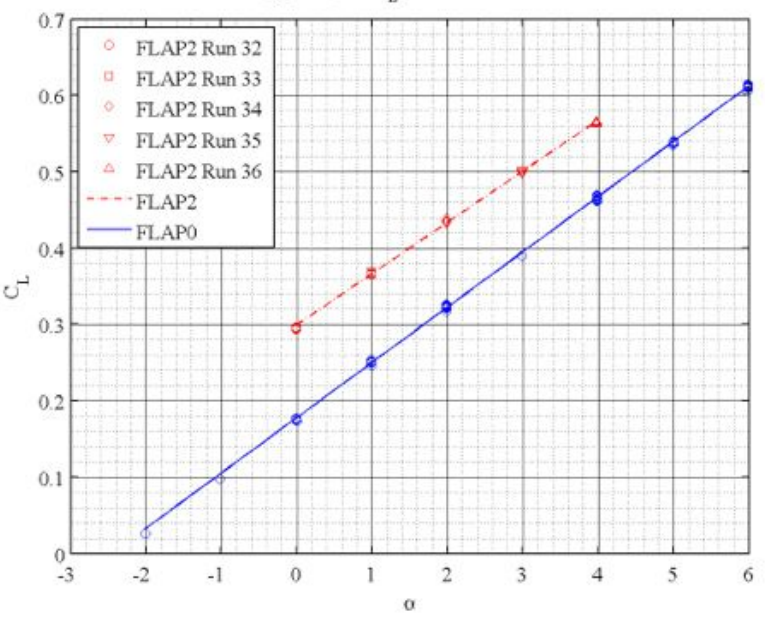

Fig. 21 - FLAP $2 C_{L}$ vs. $\alpha$

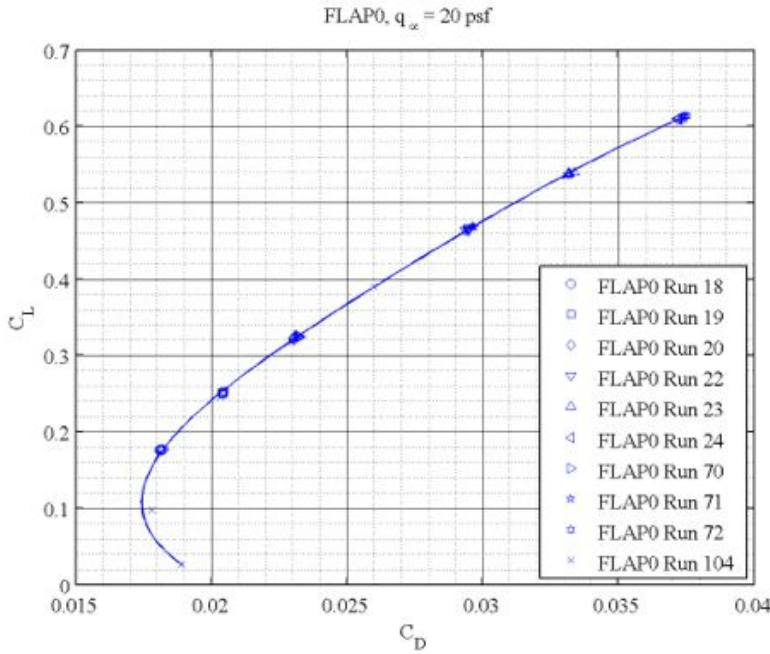

Fig. 18 - FLAP0 $C_{L}$ vs. $C_{D}$

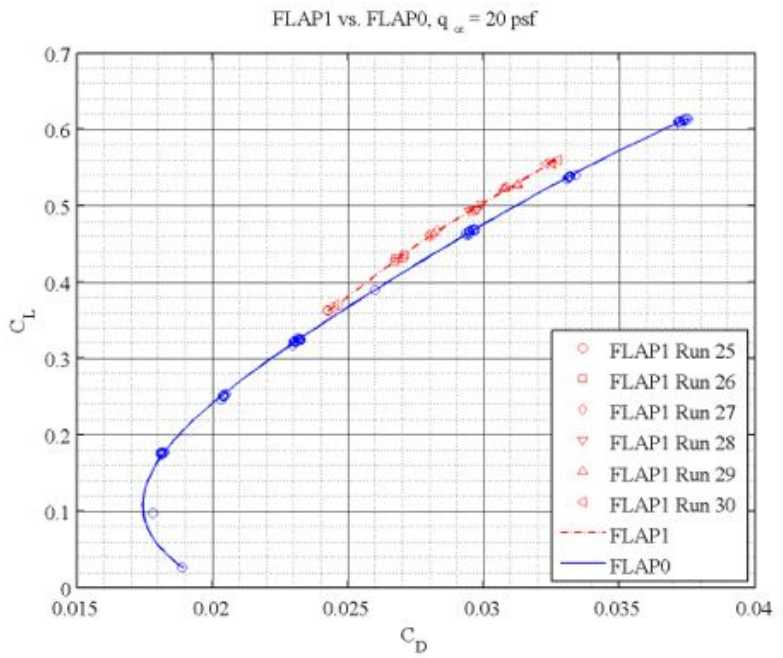

Fig. 20 - FLAP1 $C_{L}$ vs. $C_{D}$

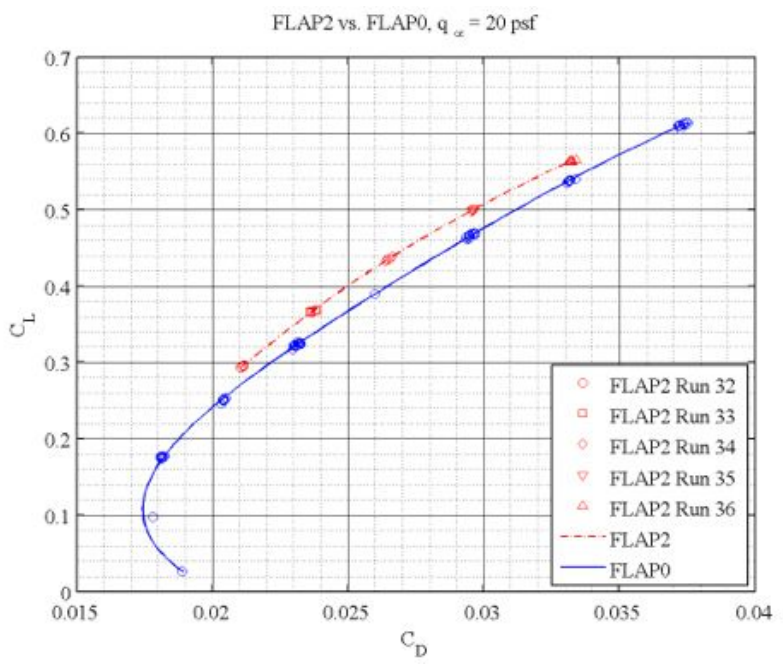

Fig. 22 - FLAP $2 C_{L}$ vs. $C_{D}$ 
FLAP3 vs. FL.AP0, $q_{\alpha}=20$ psf, $C_{L}=0.3091+3.7369 a, R=0.9995$

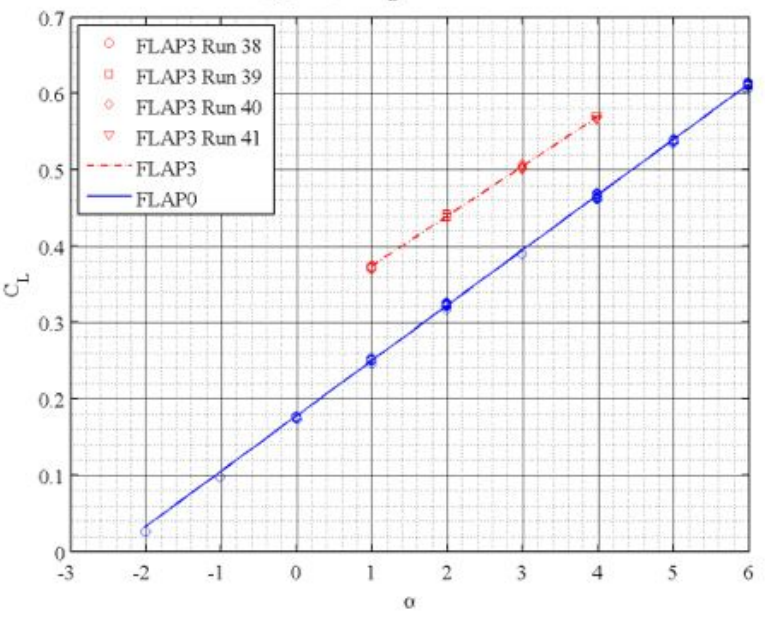

Fig. 23 - FLAP3 $C_{L}$ vs. $\alpha$

FLAP4 vs. FLAP0, $\mathrm{q}_{\alpha}=20 \mathrm{psf}, \mathrm{C}_{\mathrm{L}}=0.2776+3.8662 \alpha, \mathrm{R}=0.9998$

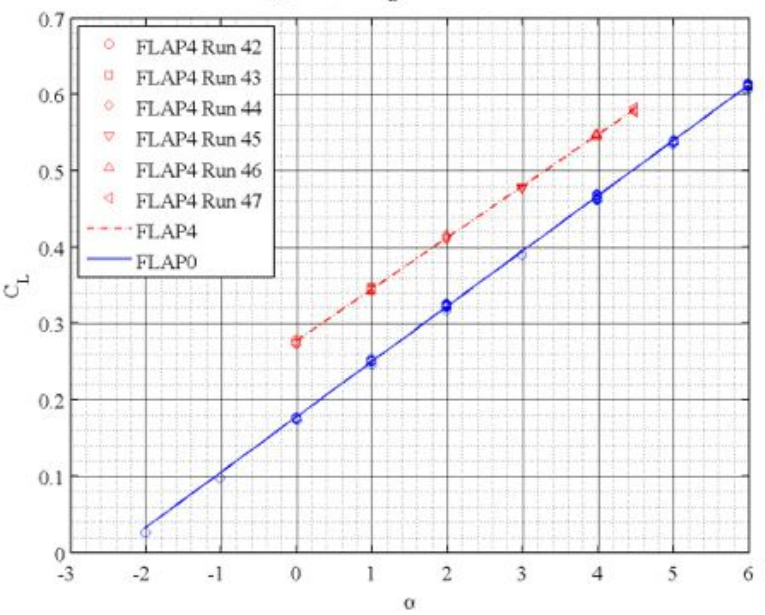

Fig. 25 - FLAP4 $C_{L}$ vs. $\alpha$

FLAP5 vs. FLAP0, $\mathrm{q}_{\alpha}=20$ psf, $\mathrm{C}_{\mathrm{L}}=0.2631+3.9121 \mathrm{o}, \mathrm{R}=0.9997$

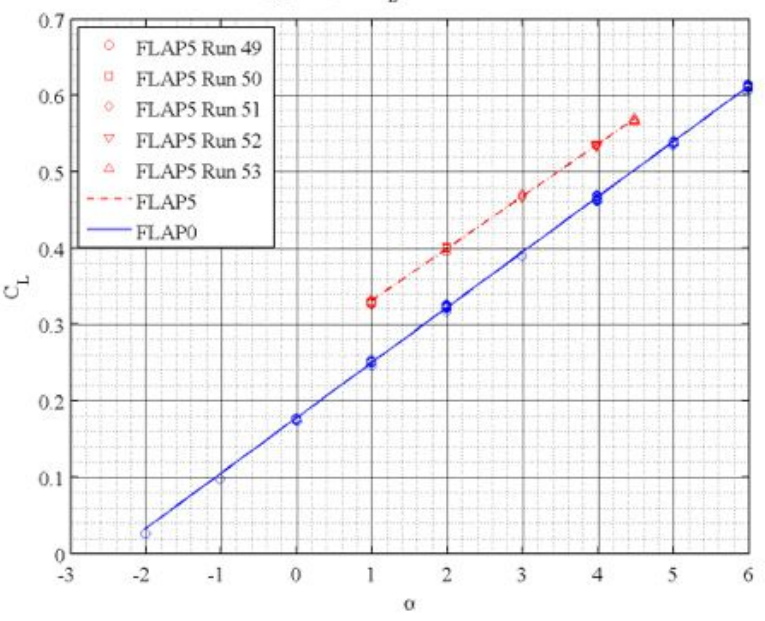

Fig. 27 - FLAP5 $C_{L}$ vs. $\alpha$

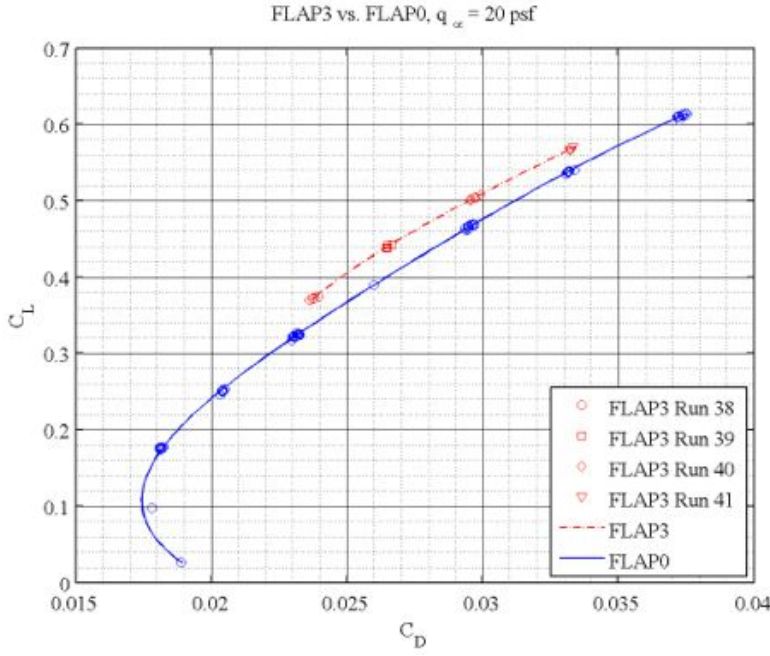

Fig. 24 - FLAP3 $C_{L}$ vs. $C_{D}$

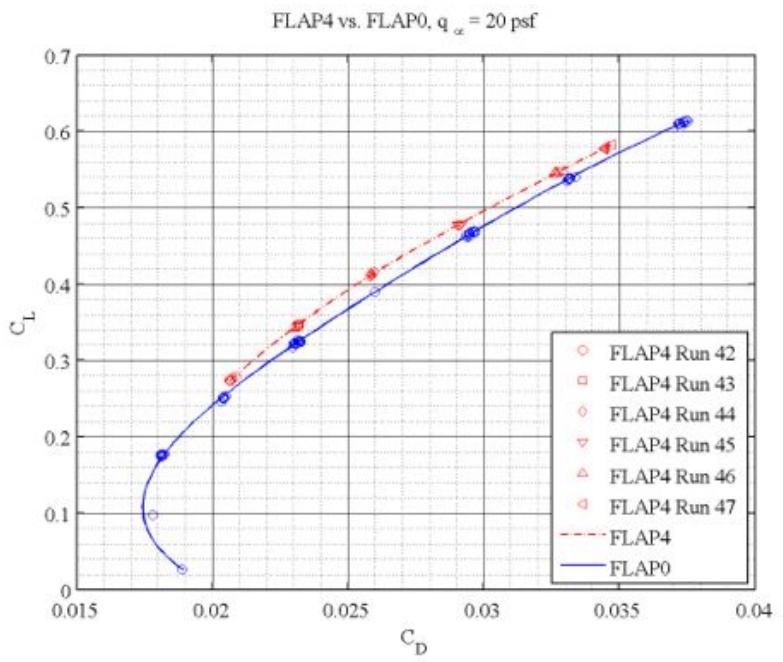

Fig. 26 - FLAP4 $C_{L}$ vs. $C_{D}$

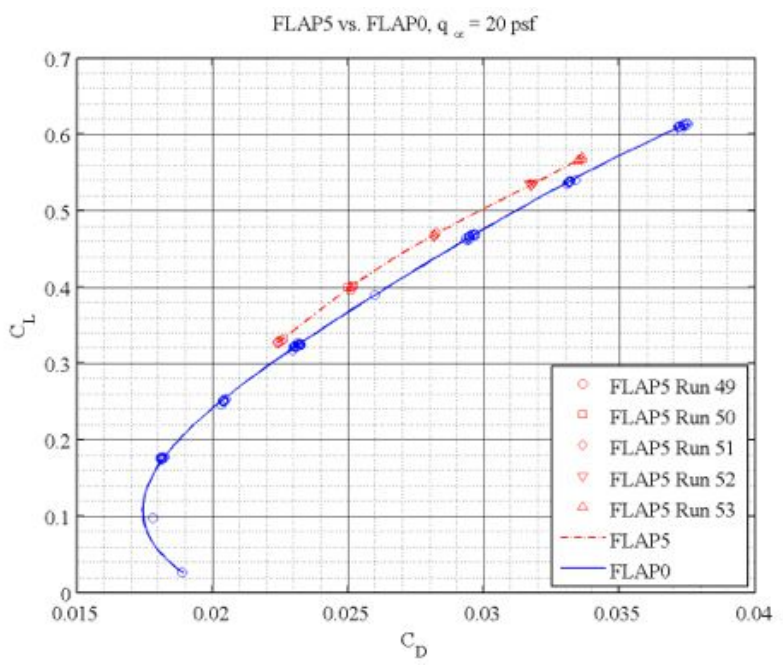

Fig. 28 - FLAP5 $C_{L}$ vs. $C_{D}$ 
FL.AP6 vs. FLAP0, $\mathrm{q}_{\alpha}=20 \mathrm{psf}, \mathrm{C}_{\mathrm{L}}=0.2045+4.0987 \mathrm{a}, \mathrm{R}=1.0000$

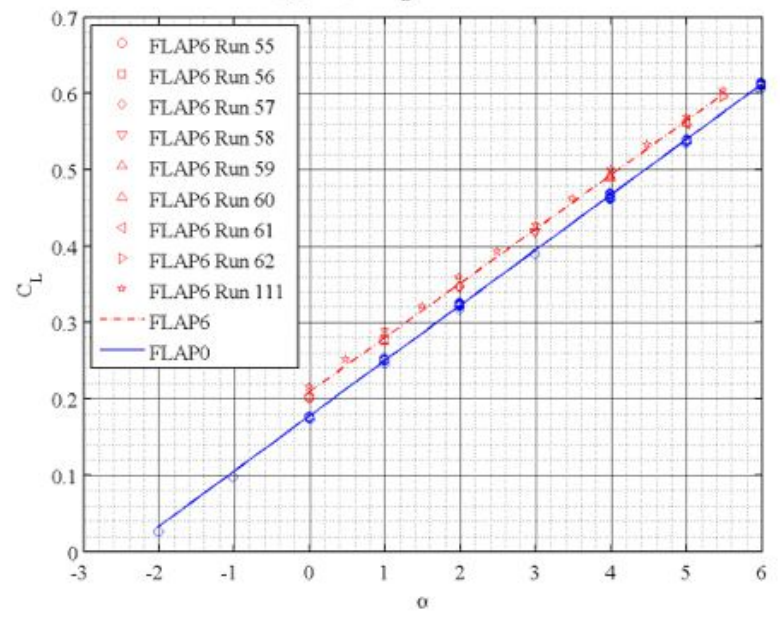

Fig. 29 - FLAP6 $C_{L}$ vs. $\alpha$

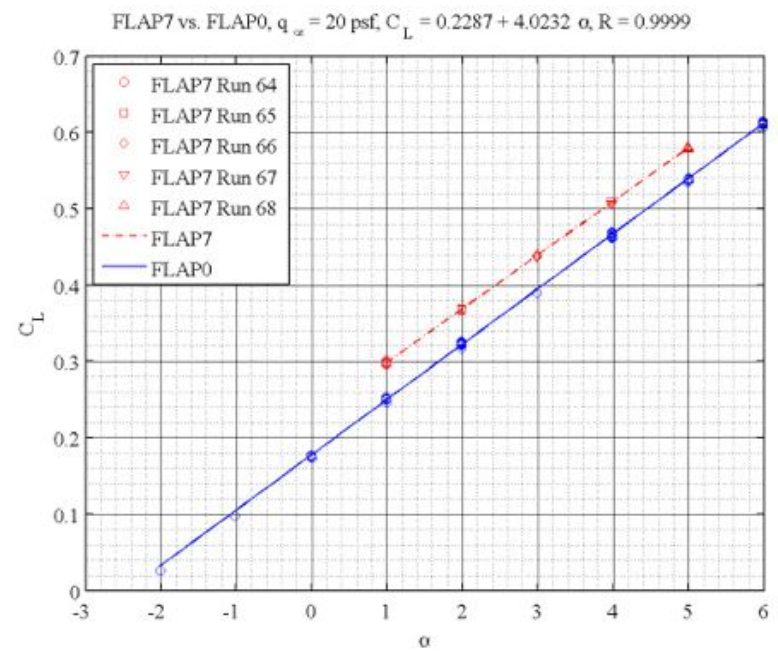

Fig. 31 - FLAP7 $C_{L}$ vs. $\alpha$

FLAP8 vs. FLAP0, $\mathrm{q}_{\alpha}=20 \mathrm{psf}, \mathrm{C}_{\mathrm{L}}=0.2235+4.0252 \mathrm{o}, \mathrm{R}=0.9999$

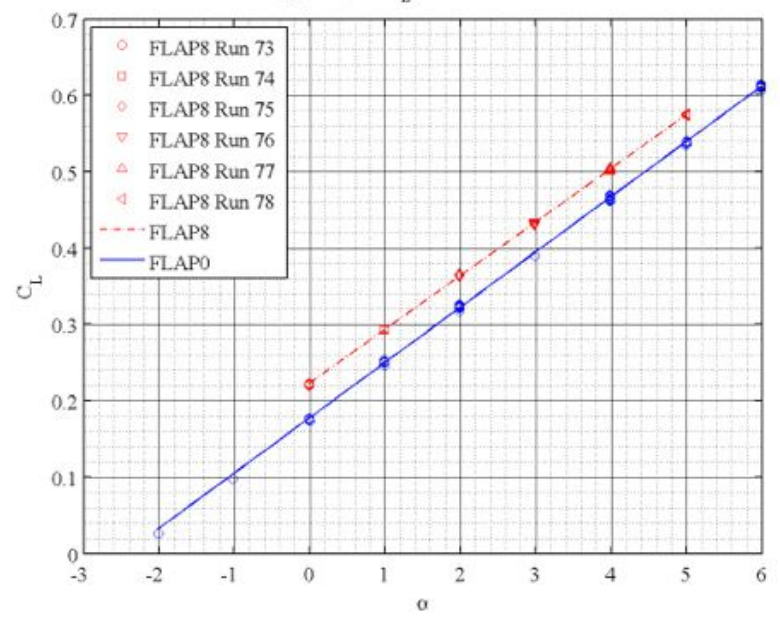

Fig. 33 - FLAP8 $C_{L}$ vs. $\alpha$

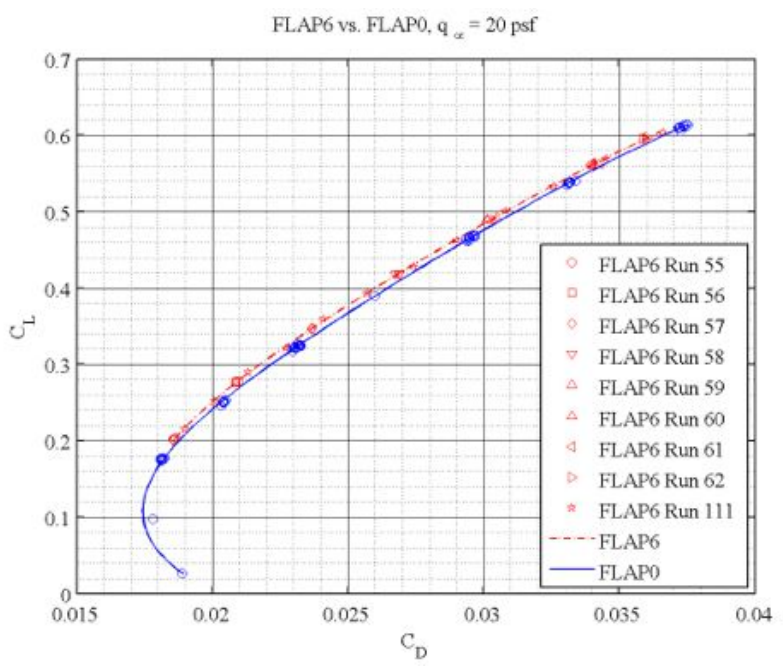

Fig. 30 - FLAP6 $C_{L}$ vs. $C_{D}$

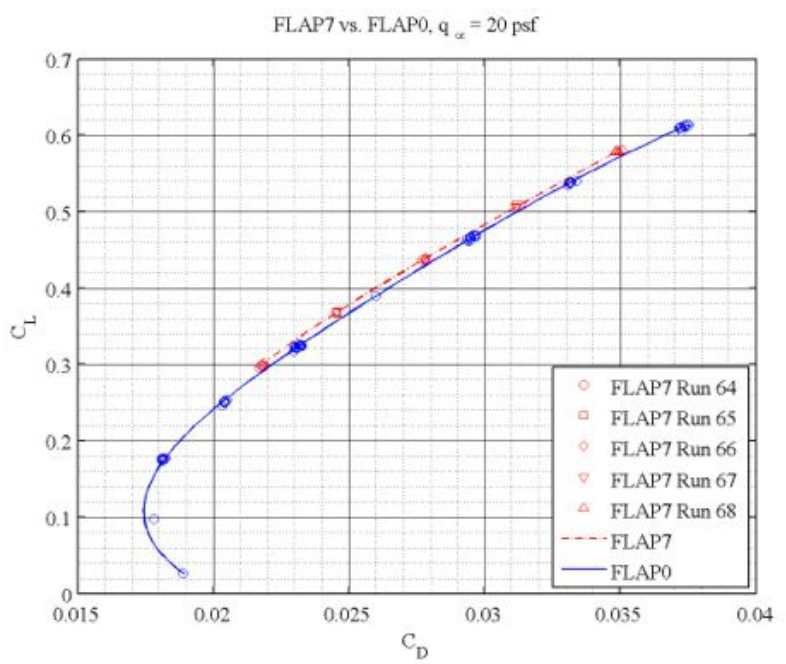

Fig. 32 - FLAP7 $C_{L}$ vs. $C_{D}$

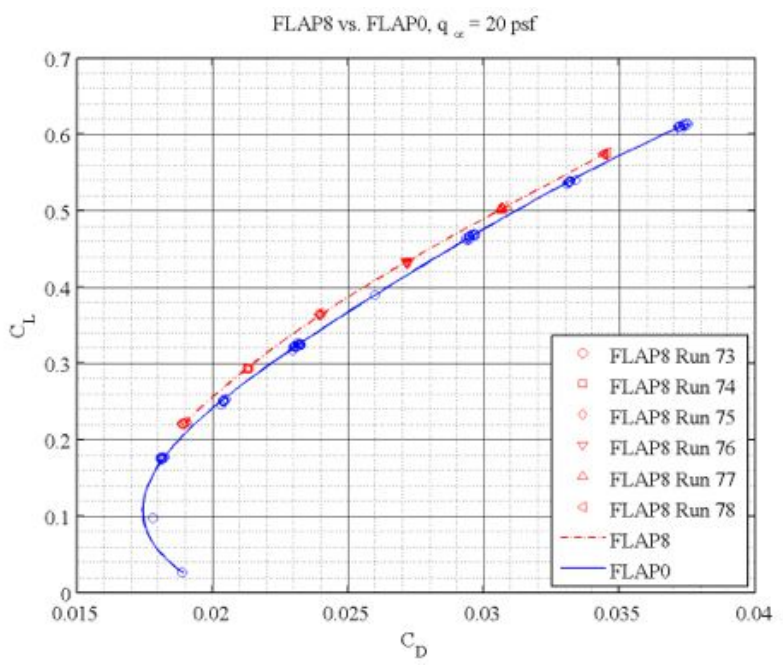

Fig. 34 - FLAP8 $C_{L}$ vs. $C_{D}$ 
FLAP9 vs. FLAP0, $\mathrm{q}_{\alpha}=20 \mathrm{psf}, \mathrm{C}_{\mathrm{L}}=0.2501+3.9606 \mathrm{\alpha}, \mathrm{R}=0.9999$

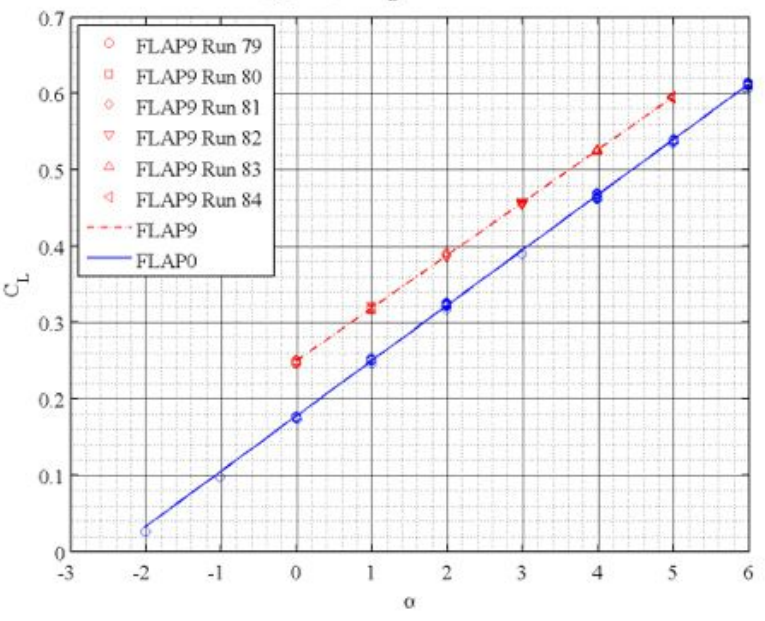

Fig. 35 - FLAP9 $C_{L}$ vs. $\alpha$

FLAP10 vs. FLAP0, $\mathrm{q}_{\alpha}=20 \mathrm{psf}, \mathrm{C}_{\mathrm{L}}=0.2398+3.9860 \alpha, \mathrm{R}=0.9999$

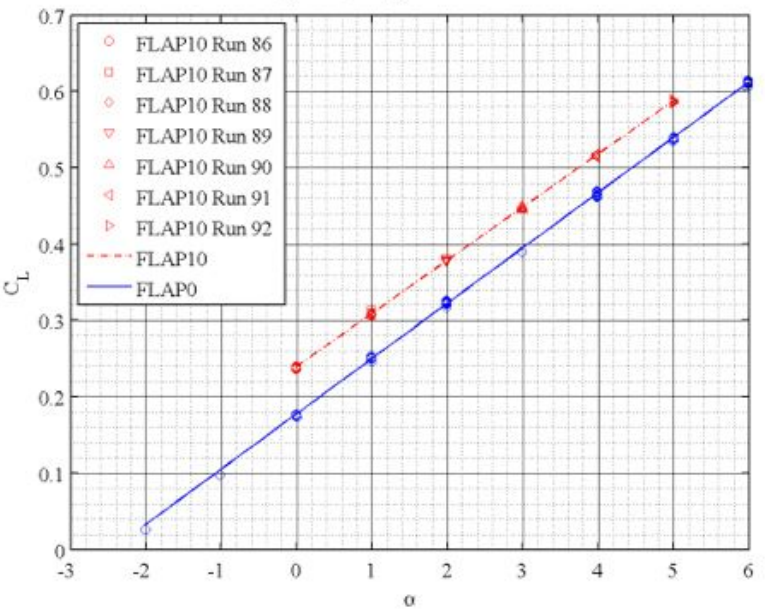

Fig. 37 - FLAP10 $C_{L}$ vs. $\alpha$

FLAP11 vs. FLAP0, $q_{\alpha}=20$ psf, $C_{L}=0.1331+4.2025 \alpha R=1.0000$

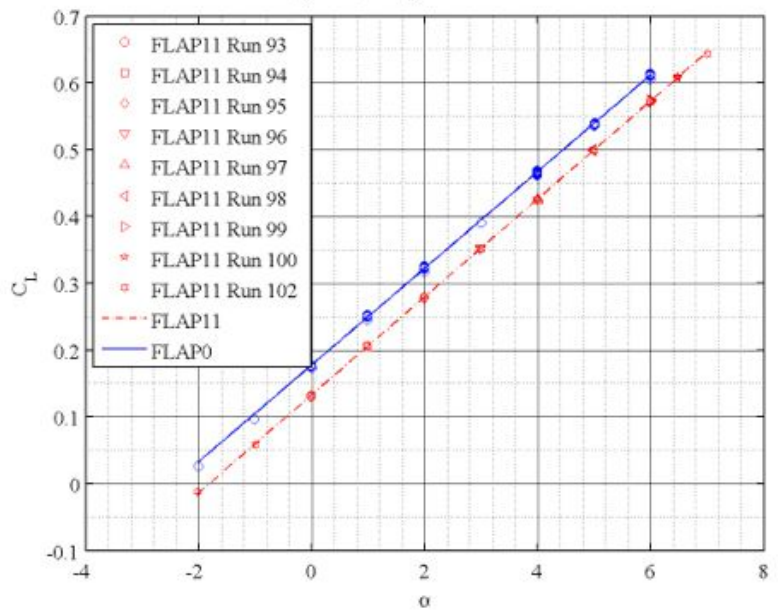

Fig. 39 - FLAP11 $C_{L}$ vs. $\alpha$

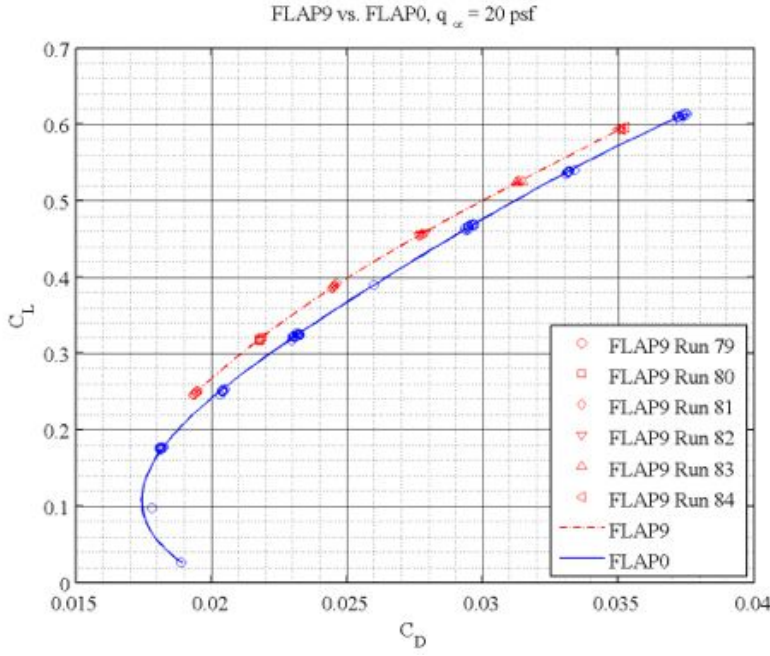

Fig. 36 - FLAP9 $C_{L}$ vs. $C_{D}$

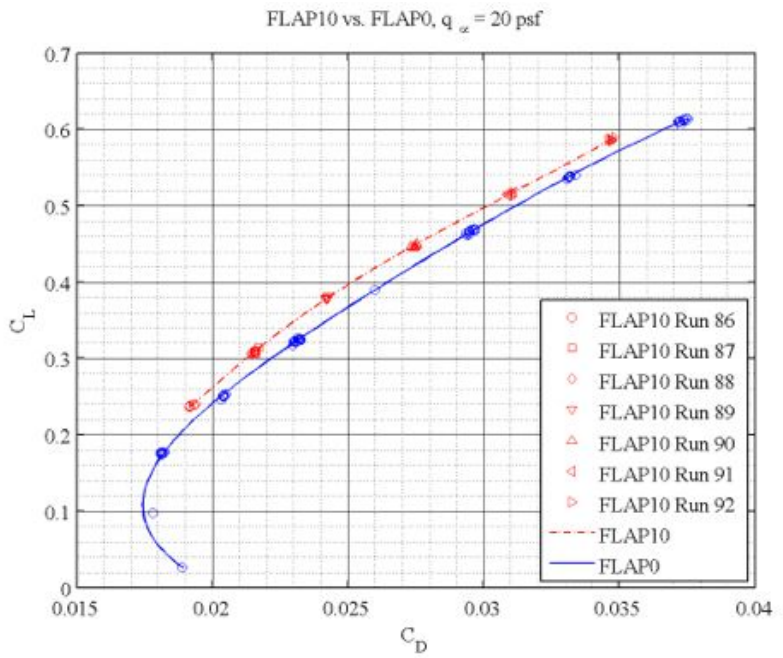

Fig. 38 - FLAP10 $C_{L}$ vs. $C_{D}$

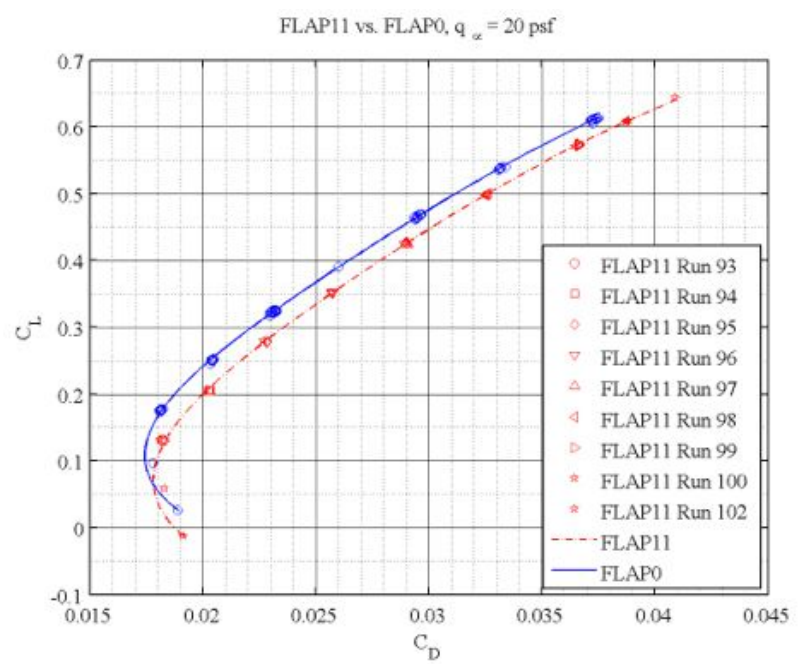

Fig. 40 - FLAP11 $C_{L}$ vs. $C_{D}$ 
FLAP1 vs. FL.AP0, $\mathrm{q}_{\alpha}=20 \mathrm{psf}, \mathrm{C}_{\mathrm{L}}=0.3419+3.9465 \mathrm{\alpha}, \mathrm{R}=0.9996$

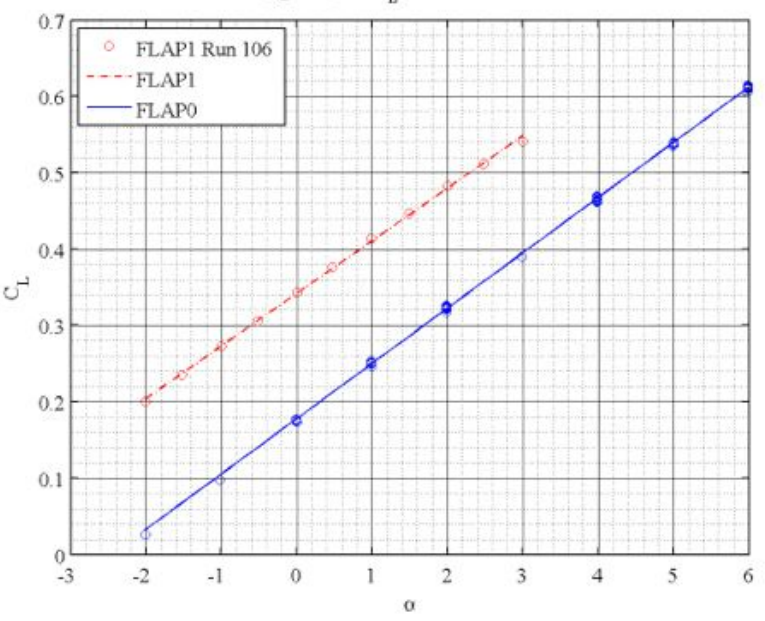

Fig. 41 - FLAP1* $C_{L}$ vs. $\alpha$

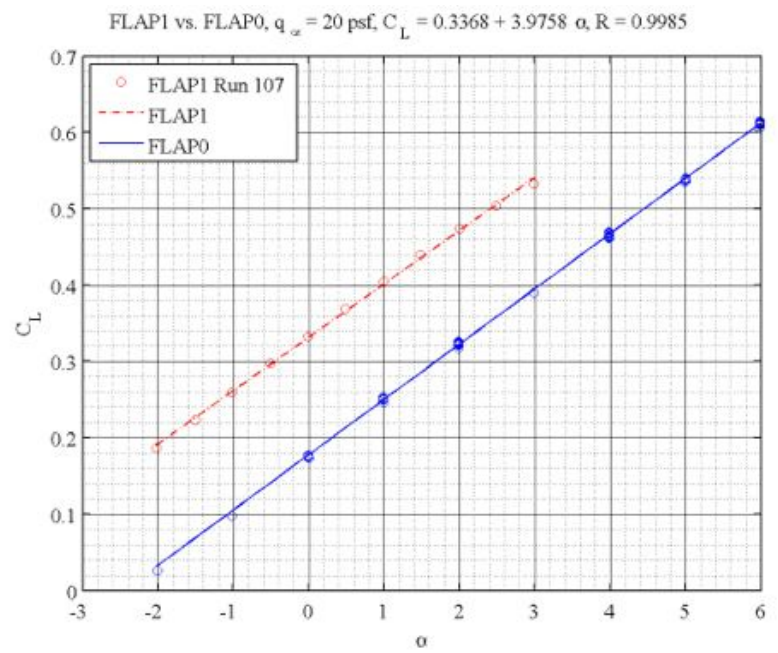

Fig. 43 - FLAP1 ${ }^{* *} C_{L}$ vs. $\alpha$

FLAP7 vs. FLAP0, $q_{\alpha}=20$ psf, $C_{L}=0.2336+4.0720 o, R=0.9999$

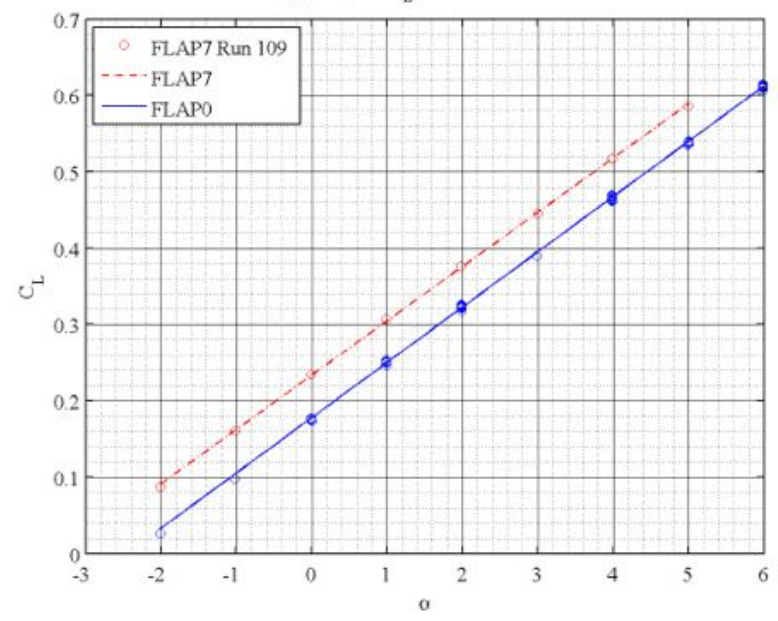

Fig. 45 - FLAP7* $C_{L}$ vs. $\alpha$

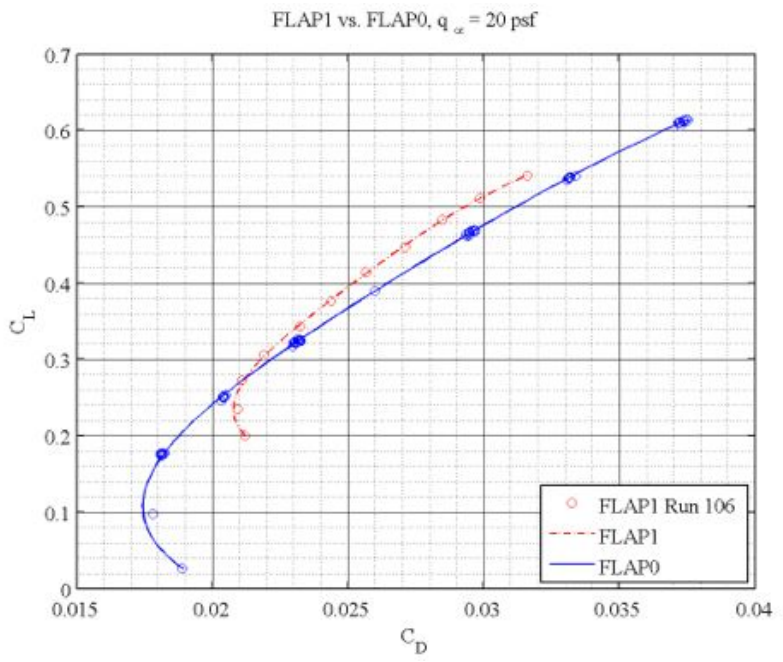

Fig. 42 - FLAP $1 * C_{L}$ vs. $C_{D}$

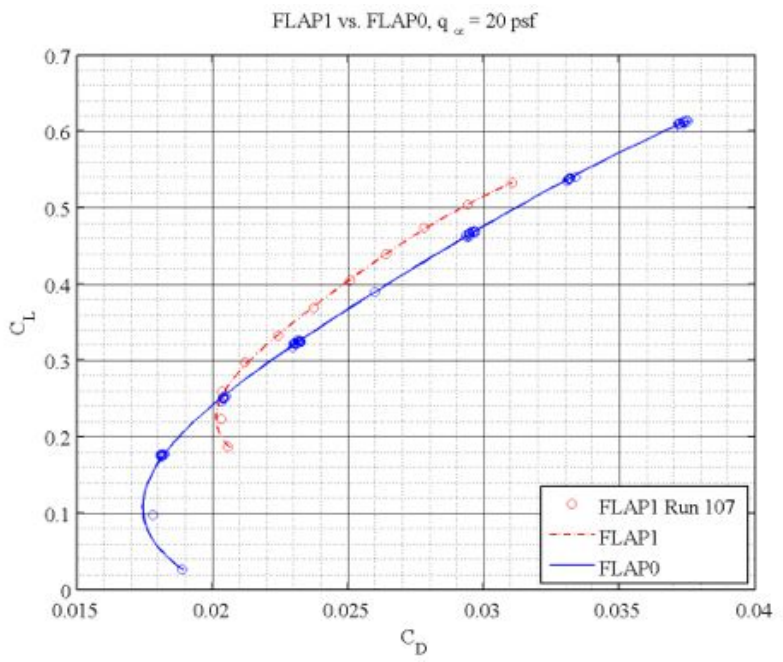

Fig. 44 - FLAP1** $C_{L}$ vs. $C_{D}$

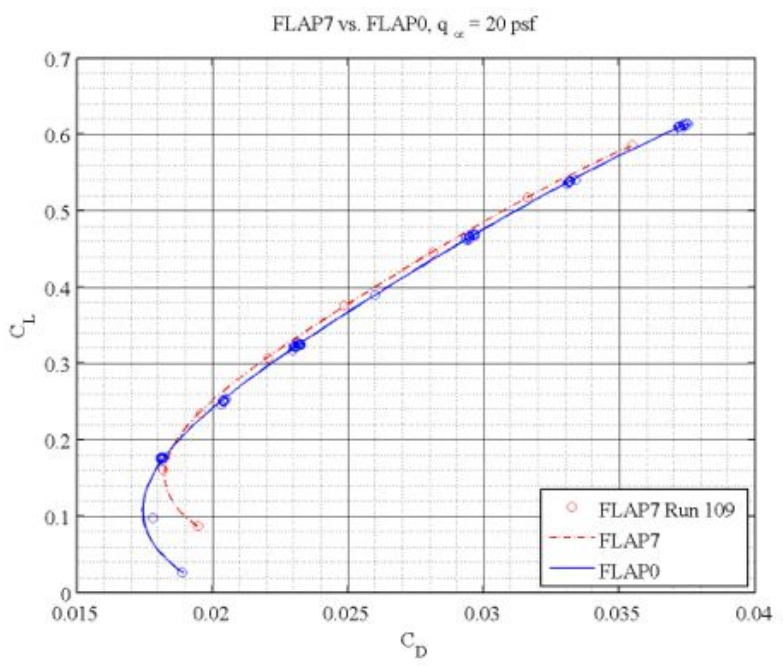

Fig. 46 - FLAP7 $* C_{L}$ vs. $C_{D}$ 


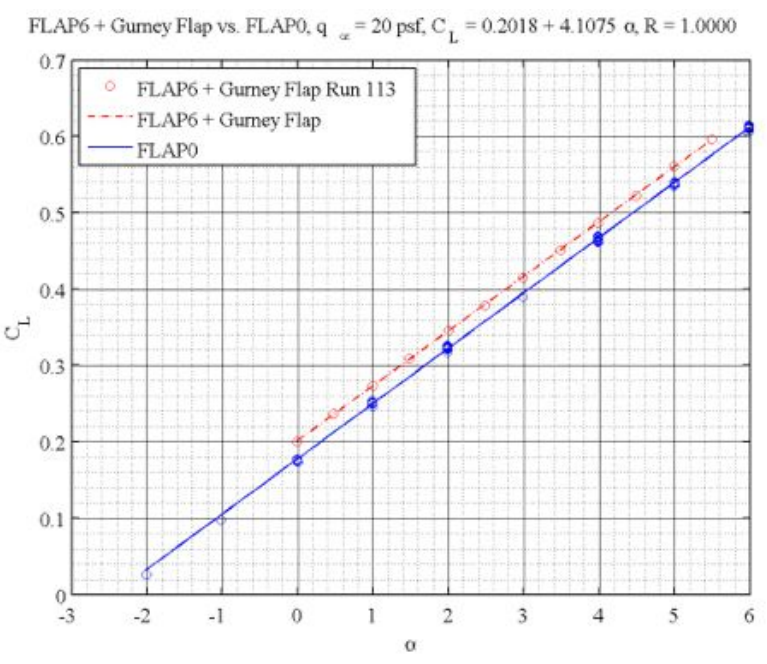

Fig. 47 - FLAP12 $C_{L}$ vs. $\alpha$

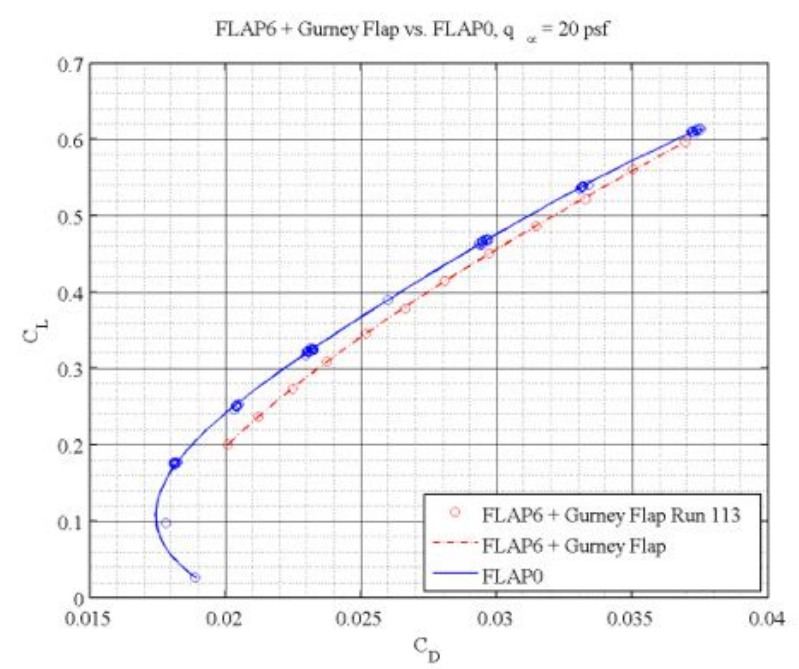

Fig. 48 - FLAP12 $C_{L}$ vs. $C_{D}$

Figures 49 and 50 are the plots of $L / D$ for FLAP0, FLAP1, FLAP7, and FLAP11 configurations. The baseline FLAP0 configuration has a $L / D$ value of about 16.1 at $C_{L}=0.51$. FLAP1 configuration has the highest $L / D$ at the same $C_{L}$ as compared to all the other VCCTEF configurations. Its $L / D$ is about 17.2 which is about a $6.8 \%$ improvement. FLAP11 configuration is not aerodynamically efficient since it is configured as a lift-reduction device with negative VCCTEF deflections.

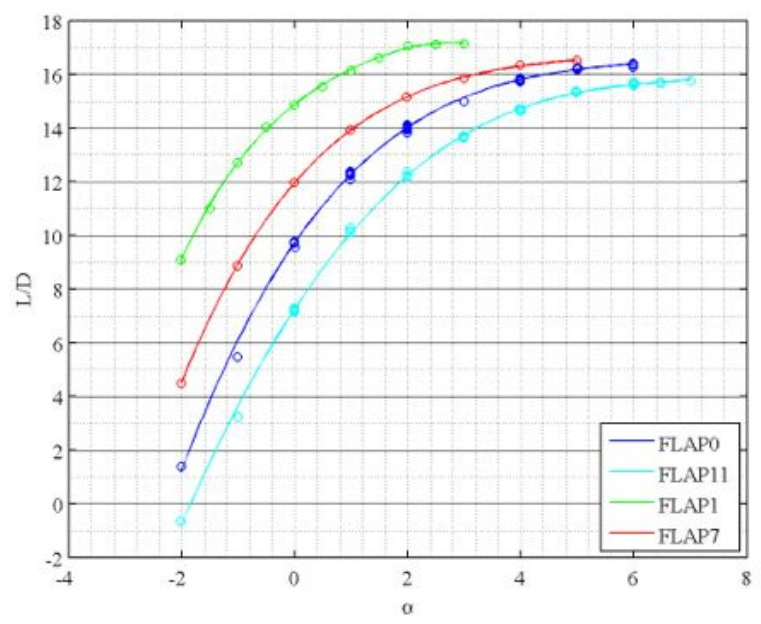

Fig. $49-L / D$ vs. $\alpha$

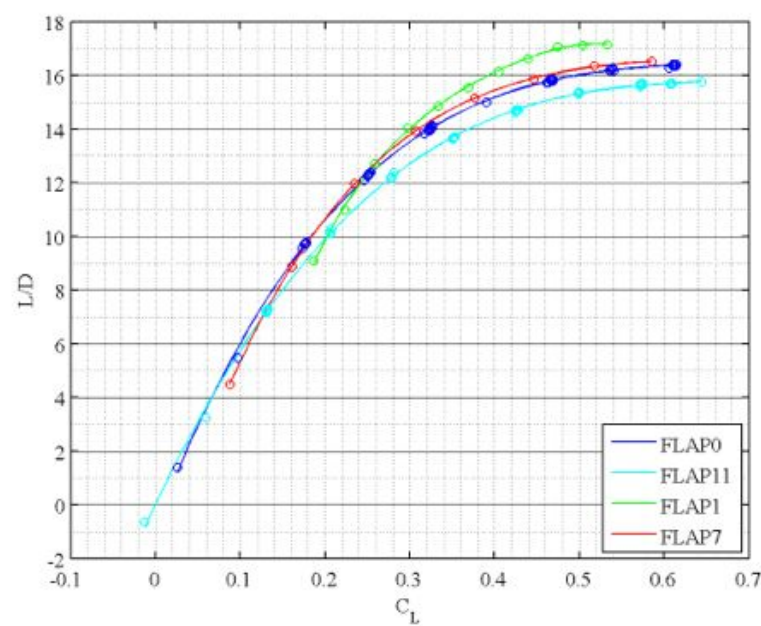

Fig. $50-L / D$ vs. $C_{L}$

Table 4 presents the summary of the drag comparison for all the VCCTEF configurations. As can be seen, FLAP1 configuration is the most aerodynamically efficient, achieving a maximum of $6.31 \%$ drag reduction at the design cruise $C_{L}$. All VCCTEF configurations except FLAP11 and FLAP12 achieve varying degree of drag reduction. In terms of the absolute values of $L / D$, the maximum increase in $L / D$ is $4.85 \%$ due to FLAP1 configuration.

Comparing FLAP2 configuration to FLAP3 configuration, increasing the VCCTEF deflection at the mid-span and tapering it off toward inboard and outboard seems to be more aerodynamically efficient than the other way around.

Comparing FLAP 4 configuration to FLAP5 configuration, increasing flap deflection toward the wing tip seems to improve aerodynamic efficiency compared to increasing flap deflection toward the wing root. This may seem counter-intuitive because of perceived increase in span load toward the wing tip in FLAP4 that would cause a lift distribution to deviate more from the ideal elliptical lift distribution. However, the effective angle of attack is a function of both rigid and flexible rotations of the wing sections. To understand how FLAP4 and FLAP5 affect $L / D$, we need to look at the combined rigid and flexible effective angle of attack distribution along the span in each case.

FLAP8 configuration is essentially a plain flap since the two outer camber segments are locked to zero deflection relative to the forward camber segment. FLAP8 configuration achieves $1.89 \%$ drag reduction and $1.73 \%$ improvement in $L / D$. FLAP9 configuration is the deflection of the trailing edge camber segment. It is rather surprising that this trailing edge camber segment 
has a significant influence on drag reduction. Compared to FLAP8 configuration, FLAP9 configuration is more aerodynamically efficient with $3.79 \%$ drag reduction and $3.45 \%$ improvement in $L / D$.

It should be noted that the observed $L / D$ improvements are applicable to the low-speed flow in the UWAL without accounting for a full-configuration with pitch trim and thrust effects. High-speed flow and the contribution of the horizontal tails as well as the nacelle interference can influence the performance of the VCCTEF. This assessment would require a full-configuration high-speed wind tunnel test.

\begin{tabular}{|c|c|c|c|c|c|}
\hline FLAP & $C_{D} @ C_{L}=0.51$ (Counts) & $C_{D}$ Reduction (Counts) & $\%$ Drag Reduction & $L / D$ Max & $\% L / D$ Increase \\
\hline \hline 0 & 317 & 0 & 0 & 16.4182 & 0 \\
\hline 1 & 303 & 14 & 4.42 & 17.2151 & 4.85 \\
\hline $1^{*}$ & 298 & 19 & 5.99 & 17.1337 & 4.36 \\
\hline $1^{* *}$ & 297 & 20 & 6.31 & 17.1553 & 4.49 \\
\hline 2 & 301 & 16 & 5.05 & 17.0012 & 3.55 \\
\hline 3 & 300 & 17 & 5.36 & 17.1285 & 4.33 \\
\hline 4 & 308 & 9 & 2.84 & 16.8104 & 2.39 \\
\hline 5 & 304 & 13 & 4.10 & 16.9709 & 3.37 \\
\hline 6 & 313 & 4 & 1.26 & 16.6054 & 1.14 \\
\hline 7 & 313 & 4 & 1.26 & 16.6340 & 1.31 \\
\hline $7 *$ & 312 & 5 & 1.58 & 16.5125 & 0.57 \\
\hline 8 & 311 & 6 & 1.89 & 16.7022 & 1.73 \\
\hline 9 & 305 & 12 & 3.79 & 16.9840 & 3.45 \\
\hline 10 & 307 & 10 & 3.15 & 16.9420 & 3.19 \\
\hline 11 & 331 & -14 & -4.42 & 15.7418 & -4.12 \\
\hline 12 & 326 & -9 & -2.84 & 16.1385 & -1.70 \\
\hline
\end{tabular}

Table 4 - Summary of Drag Reduction an $L / D$ Improvements of VCCTEF (1 Drag Count $=0.0001)$

Runs 116 to 125 were designed to examine the effect of the dynamic pressure on lift and drag for FLAP0 and FLAP7 configurations. The interpolated test data for $\alpha=-2^{\circ}, 0^{\circ}, 2^{\circ}, 4^{\circ}$ are plotted in Figs. 51 to 54 . The results show that lift and drag are decreasing as the dynamic pressure increases. The data support the foregoing aeroelastic analysis, which shows that lift coefficient decreases with increasing dynamic pressure. The third-order dependency of $C_{L}$ on $q_{\infty}$ is predominantly dictated by the linear term, which is due to $K_{s}^{-1}$ in Eq. (41) since this term is larger than the other two terms. Note that the test runs were conducted at low dynamic pressures relative to the divergence dynamic pressure. For such low dynamic pressures, the accuracy of the series approximation in powers of the dynamic pressure is acceptable.

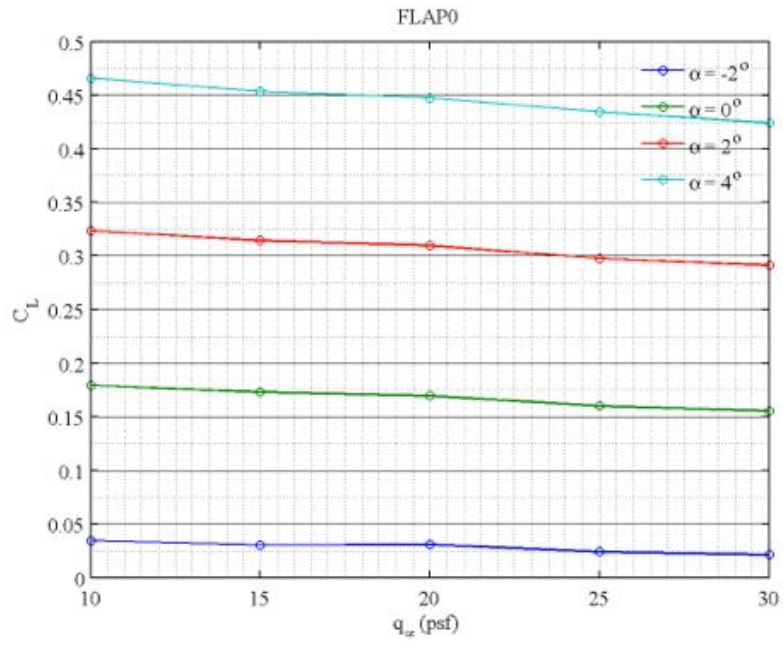

Fig. 51 - FLAP0 $C_{L}$ vs. $q_{\infty}$

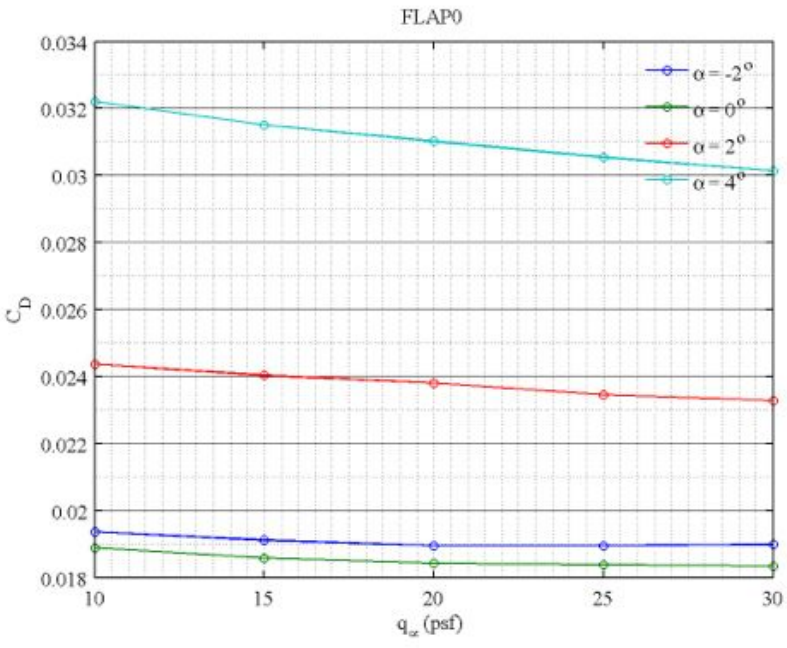

Fig. 52 - FLAP0 $C_{D}$ vs. $q_{\infty}$ 


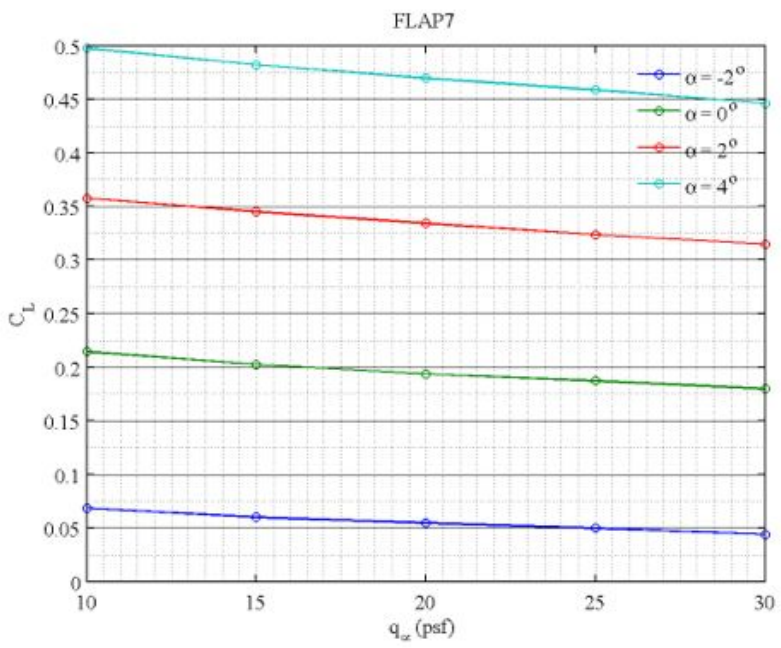

Fig. 53 - FLAP7 $C_{L}$ vs. $q_{\infty}$

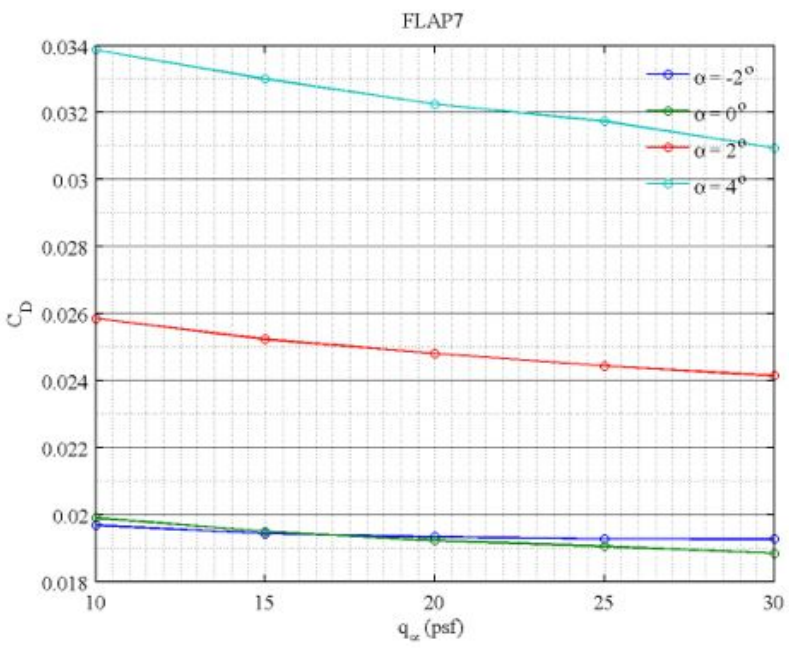

Fig. 54 - FLAP7 $C_{D}$ vs. $q_{\infty}$

The rigid-wing lift coefficient can be estimated from the dynamic pressure data. Using a cubic variation of $C_{L}$ with $q_{\infty}$ as derived from the aeroelastic analysis, the lift coefficient for FLAP0 configuration can be estimated as

$$
\begin{aligned}
C_{L} & =0.1866+4.3892 \alpha+\left(1.8731 \times 10^{-7} q_{\infty}^{3}-1.9561 \times 10^{-5} q_{\infty}^{2}-6.1839 \times 10^{-4} q_{\infty}\right) \\
& +\left(-2.2077 \times 10^{-5} q_{\infty}^{3}+1.3363 \times 10^{-3} q_{\infty}^{2}-3.8151 \times 10^{-2} q_{\infty}\right) \alpha
\end{aligned}
$$

Therefore, the rigid-wing lift coefficient of FLAP0 configuration is estimated to be

$$
C_{L}^{*}=0.1866+4.3892 \alpha
$$

This rigid-wing lift coefficient is contrasted to the lift coefficient of the flexible wing at $q_{\infty}=20$ psf for run 116

$$
C_{L}=0.1679+3.9841 \alpha
$$

For FLAP7 configuration, the lift coefficient is estimated as

$$
\begin{aligned}
C_{L} & =0.2502+4.1760 \alpha+\left(-2.2090 \times 10^{-6} q_{\infty}^{3}+1.5512 \times 10^{-4} q_{\infty}^{2}-5.0359 \times 10^{-3} q_{\infty}\right) \\
& +\left(8.8389 \times 10^{-6} q_{\infty}^{3}-5.0515 \times 10^{-4} q_{\infty}^{2}-3.8331 \times 10^{-3} q_{\infty}\right) \alpha
\end{aligned}
$$

The rigid-wing lift coefficient of FLAP7 configuration is estimated to be

$$
C_{L}^{*}=0.2502+4.1760 \alpha
$$

This is compared to the lift coefficient of the flexible wing at $q_{\infty}=20$ psf for run 123

$$
C_{L}=0.1938+3.9680 \alpha
$$

The rigid incremental lift coefficient of FLAP7 configuration from the baseline FLAP0 configuration is

$$
\Delta C_{L}^{*}=0.0636-0.2132 \alpha
$$

The aeroelastic incremental lift coefficient of FLAP7 configuration from the baseline FLAP0 configuration is computed by subtracting the $q_{\infty}$-dependent term in Eq. (59) from that in Eq. (56)

$$
\begin{aligned}
\Delta C_{L} & =\left(-2.3963 \times 10^{-6} q_{\infty}^{3}+1.7468 \times 10^{-4} q_{\infty}^{2}-4.4176 \times 10^{-3} q_{\infty}\right) \\
& +\left(3.0915 \times 10^{-5} q_{\infty}^{3}-1.8414 \times 10^{-3} q_{\infty}^{2}+3.4318 \times 10^{-2} q_{\infty}\right) \alpha
\end{aligned}
$$

The resulting aeroelastic incremental lift coefficient of FLAP7 configuration is negative within the range of the angle of attack and dynamic pressure in the wind tunnel test. Control reversal occurs when $\Delta C_{L}=-\Delta C_{L}^{*}$. For $\alpha=0$, this occurs at $q_{\infty}=45 \mathrm{psf}$. As the angle of attack increases, the dynamic pressure at which control reversal occurs increases significantly. 
Due to the nature of data extrapolation which can introduce uncertainty, it is of interest to examine the linear variation of $C_{L}$ with $q_{\infty}$. For FLAP0 configuration, the lift coefficient is then estimated to be

$$
C_{L}=0.1907+4.2460 \alpha+\left(-1.1601 \times 10^{-3}-1.3068 \times 10^{-2} \alpha\right) q_{\infty}
$$

Then, the rigid-wing lift coefficient of FLAP0 configuration is

$$
C_{L}^{*}=0.1907+4.2460 \alpha
$$

Comparing this expression to that estimated by the cubic variation of $C_{L}$ with $q_{\infty}$, the agreement is very good as shown in Fig. 55. The cubic extrapolation seems to produce a higher lift curve slope as shown in Fig. 57.

The estimated lift coefficient as a function of $q_{\infty}$ and rigid-wing coefficient for FLAP7 configuration based on a linear variation of $C_{L}$ with $q_{\infty}$ are obtained as

$$
\begin{aligned}
& C_{L}=0.2283+4.2229 \alpha+\left(-1.6696 \times 10^{-3}-1.2681 \times 10^{-2} \alpha\right) q_{\infty} \\
& C_{L}^{*}=0.2283+4.2229 \alpha
\end{aligned}
$$

Comparing the rigid-wing lift coefficients for FLAP7 configuration estimated by the cubic and linear variations of $C_{L}$ with $q_{\infty}$, the cubic extrapolation consistently produces higher estimates of the rigid-wing lift coefficients at angles of attack from $-2^{\circ}$ to $4^{\circ}$, as shown in Fig. 56. The discrepancy between the two estimation methods does introduce some uncertainty in the estimation of the rigid-wing lift coefficient for FLAP7 configuration.

Using the lift coefficient as a function of $\alpha$ and $q_{\infty}$, one can correct for the lift coefficient for a flexible wing wind tunnel model. Applying the two $q_{\infty}$-correction methods using the cubic and linear variation of $C_{L}$ with $q_{\infty}$ to the lift curves for FLAP0 and FLAP7 configurations from runs 116 to 125, we get the estimated rigid-wing lift curves are shown in Figs. 57 and 58.

It is noted that FLAP0 and FLAP7 configurations for run 116 and 123, respectively, at $q_{\infty}=20 \mathrm{psf}$ for some reason produce noticeably different lift curves from FLAP0 and FLAP7 configurations from the earlier runs. This is shown in Figs. 59 and 60. In particular, the lift curve for FLAP7 configuration from run 123 is significantly different from that from runs 64 to 68. This discrepancy will be examined in further detail in the future. Assuming that the dependency of $C_{L}$ on $q_{\infty}$ from runs 116 to 125 is applicable to the data from the earlier runs in light of the significant difference in the lift curves, especially for FLAP7 configuration, then the $q_{\infty}$-correction method can be applied to the lift curves for FLAP0 and FLAP7 configurations in Figs. 17 and 31 to obtain the estimated rigid-wing lift curves as shown in Figs. 59 and 60.

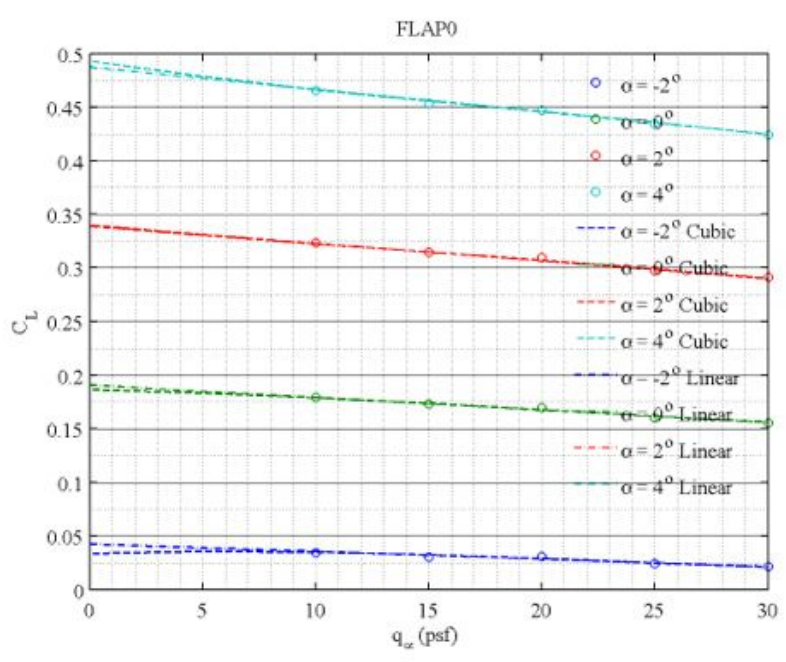

Fig. 55 - FLAP0 Cubic and Linear Variations of $C_{L}$ with $q_{\infty}$

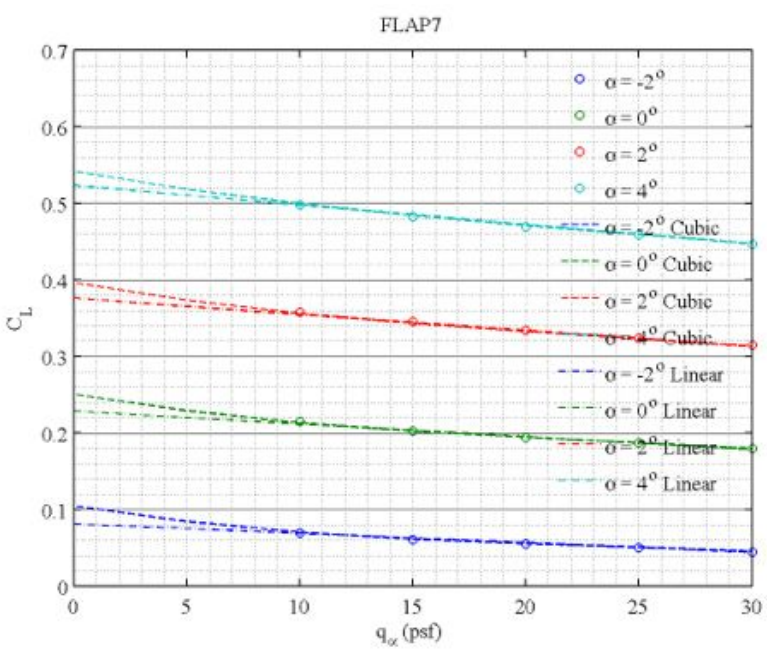

Fig. 56 - FLAP7 Cubic and Linear Variations of $C_{L}$ with $q_{\infty}$ 


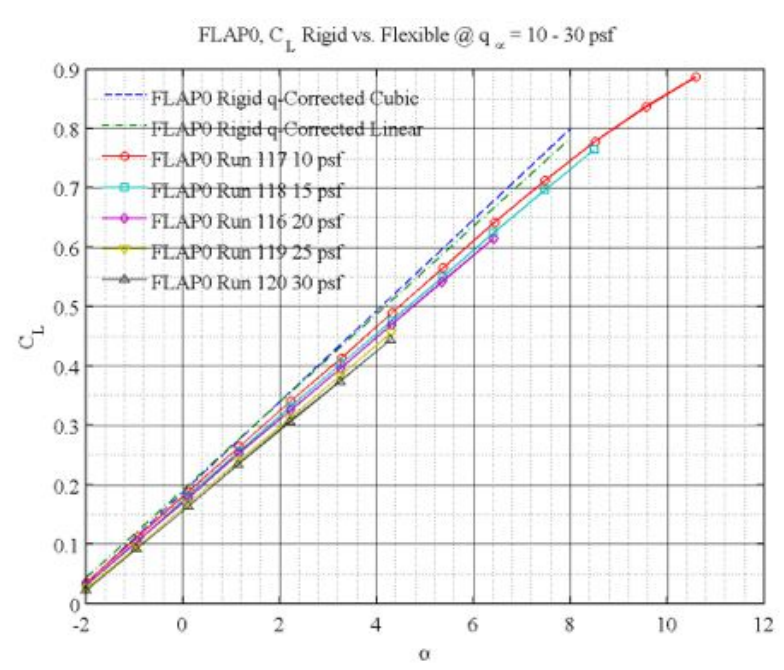

Fig. 57 - FLAP0 Runs $116-120 C_{L}$ of Flexible and Rigid Wing

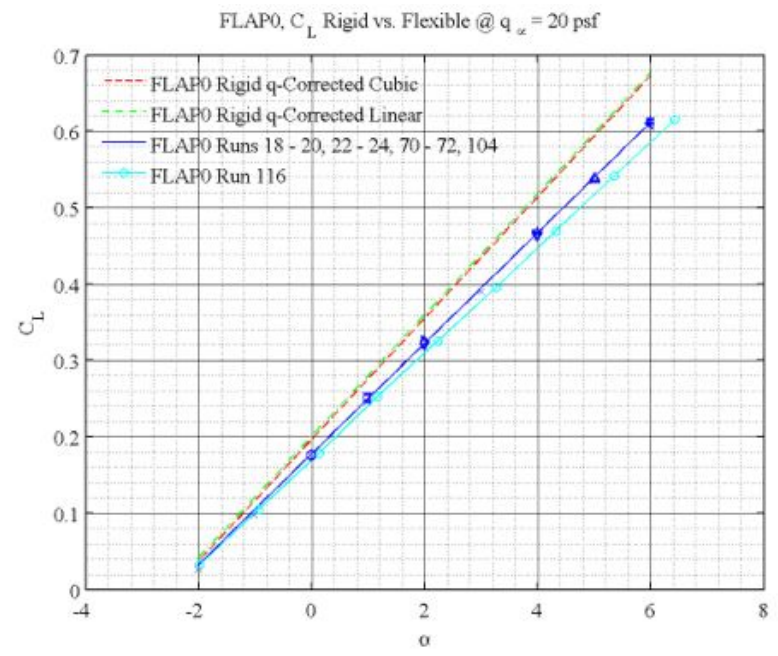

Fig. 59 - FLAP0 Runs 18 - 20, 22 - 24, $70-72,104 C_{L}$ of Flexible and Rigid Wing

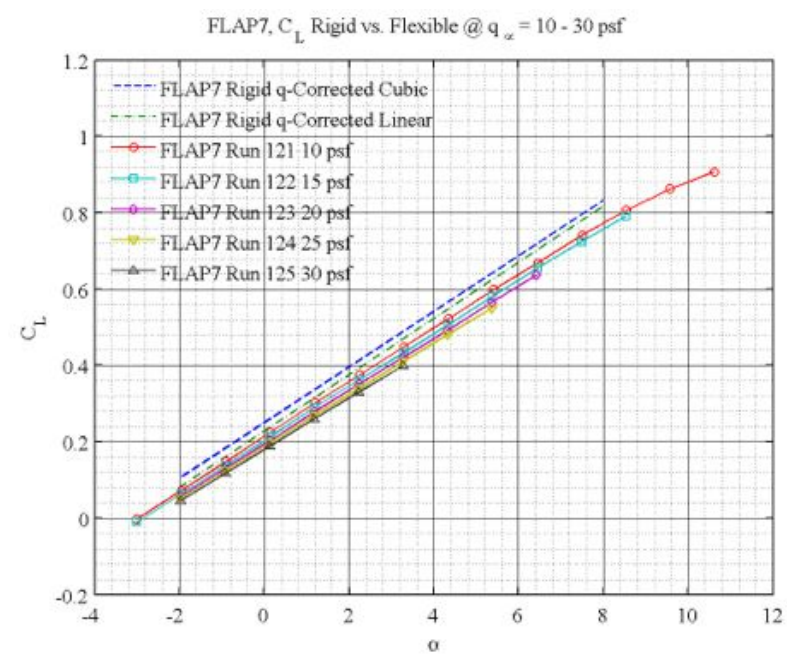

Fig. 58 - FLAP7 Runs $121-125 C_{L}$ of Flexible and Rigid Wing

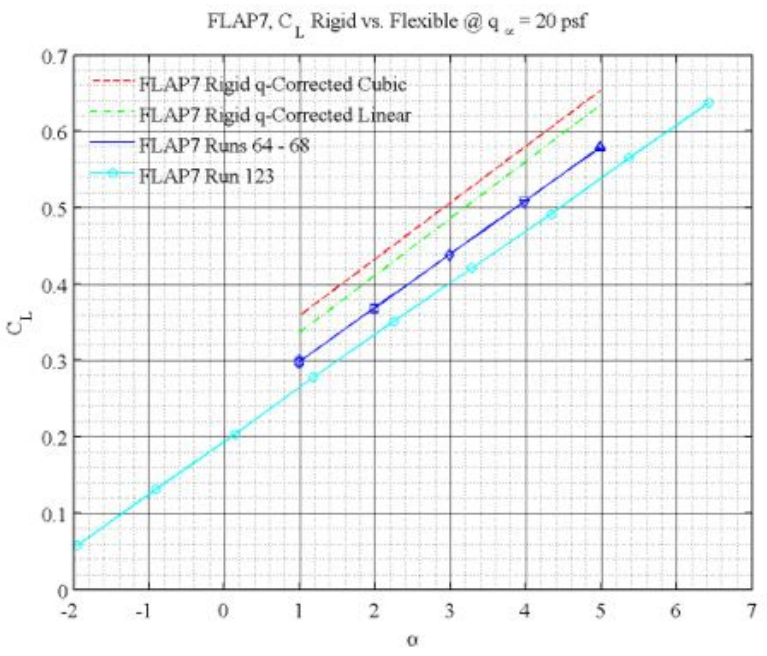

Fig. 60 - FLAP7 Runs 64 - $68 C_{L}$ of Flexible and Rigid Wing

\section{Aeroelastic Deflection Data Analysis}

Aeroelastic deflection measurements were taken with the VICON motion tracking system. The VICON system uses 54 optical targets, called dots, to create a three-dimensional displacement of the wind tunnel model as it is loaded. Figure 61 is a photograph of the wind tunnel model with the VICON dots. The displacement measurements at these dots are reduced to the spanwise out-of-plane deflections and twists at six spanwise locations, referred to as Chord 1, Chord 2, Chord 3, Chord 4, Chord 5, and Chord 6, as shown in Fig. 62. The deflection measurement locations are referenced to the origin of the wind tunnel model which starts at the intersection of the wing trailing edge and the center body. 


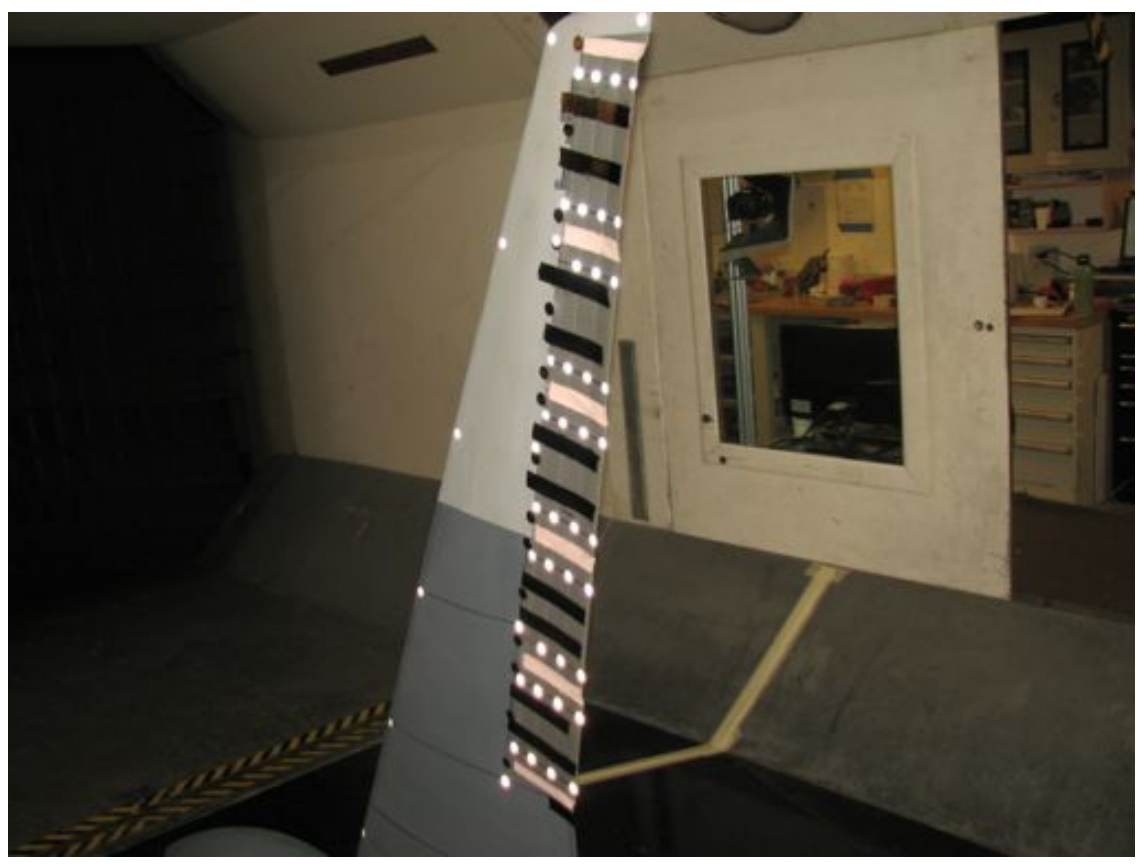

Fig. 61 - Wind Tunnel Model with VICON Dots in UWAL Test Section (Courtesy of University of Washington)

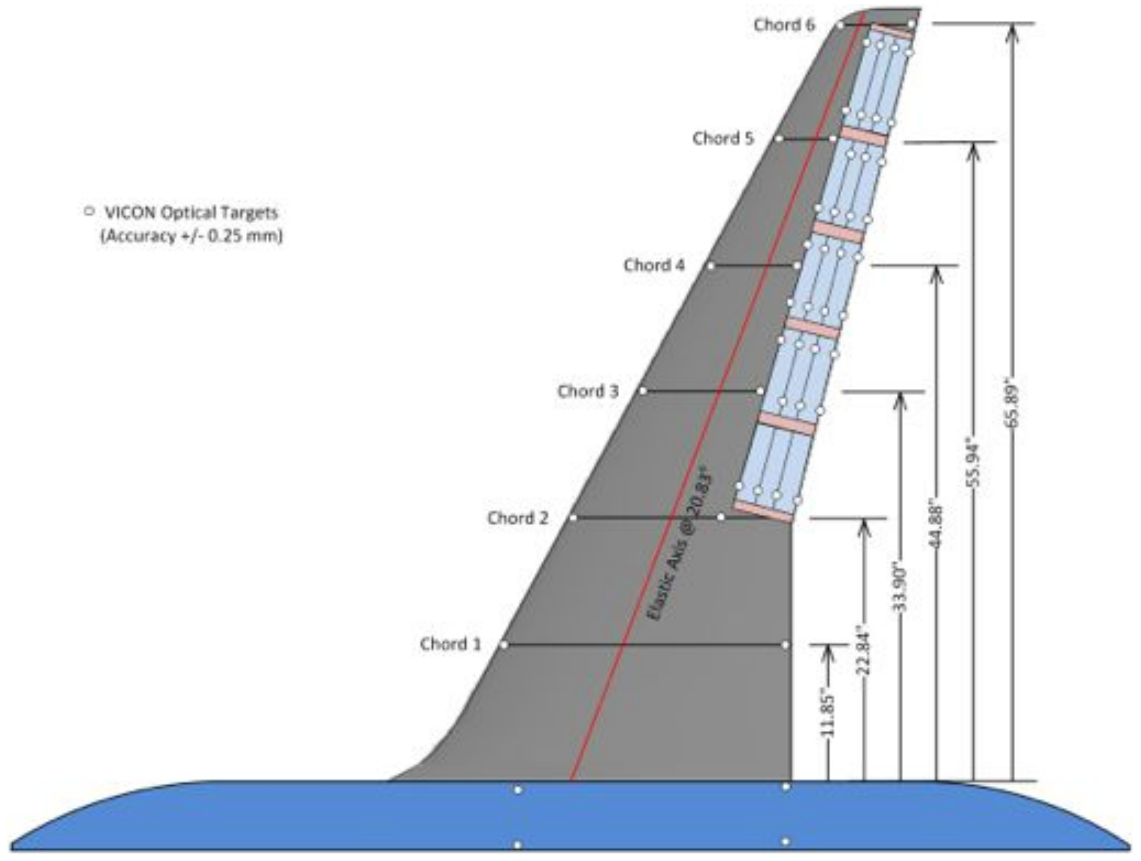

Fig. 62 - VICON Target and Spanwise Deflection Measurement Locations

The aeroelastic deflections can provide useful information on the influence of aeroelasticity on the aerodynamics of the wind tunnel model. In particular, using the aeroelastic deflections, it is possible that the rigid-wing lift coefficient of the wind tunnel model can be estimated. The static bending and torsional mode shapes in Eqs. (24) and (21) can be estimated from Galerkin's method by minimizing the cost function

$$
\begin{aligned}
J_{\Theta} & =\sum_{i=1}^{n}\left[\Theta_{i}-\Psi\left(\bar{y}_{i}\right) \theta\right]^{2} \\
J_{W} & =\sum_{i=1}^{n}\left[W_{i}-\Phi\left(\bar{y}_{i}\right) w\right]^{2}
\end{aligned}
$$


where $\Theta_{i}$ and $W_{i}$ are the torsional and bending deflection measurements at the location $\bar{y}=\bar{y}_{i}$, where $i=1, \ldots, n$ and $n$ is the number of measurements.

Consider the static torsional mode shape estimation. Minimizing the cost function yields

$$
\frac{\partial J_{\Theta}}{\partial \theta}=2 \sum_{i=1}^{n} \Psi^{\top}\left(\bar{y}_{i}\right)\left[\Theta_{i}-\Psi\left(\bar{y}_{i}\right) \theta\right]=0
$$

Then the generalized coordinates $\theta$ can be solved as a linear regression problem

$$
\boldsymbol{\theta}=\left[\sum_{i=1}^{n} \Psi^{\top}\left(\bar{y}_{i}\right) \Psi\left(\bar{y}_{i}\right)\right]^{-1} \sum_{i=1}^{n} \Psi^{\top}\left(\bar{y}_{i}\right) \Theta_{i}
$$

The deflection measurements for FLAP0 configuration from runs 18, 19, 20, 23, and 24 are presented in Table 5.

\begin{tabular}{|c|c|c|c|c|c|c|c|c|c|c|c|c|}
\hline$\alpha$ & $W_{1}$ & $W_{2}$ & $W_{3}$ & $W_{4}$ & $W_{5}$ & $W_{6}$ & $\Delta \alpha_{1}$ & $\Delta \alpha_{2}$ & $\Delta \alpha_{3}$ & $\Delta \alpha_{4}$ & $\Delta \alpha_{5}$ & $\Delta \alpha_{6}$ \\
\hline \hline 0 & 0.078 & 0.222 & 0.488 & 0.903 & 1.398 & 1.898 & -0.04 & -0.24 & -0.68 & -0.84 & -1.02 & -1.23 \\
\hline 1 & 0.076 & 0.301 & 0.713 & 1.33 & 2.064 & 2.808 & -0.16 & -0.48 & -0.87 & -0.95 & -1.35 & -1.17 \\
\hline 2 & 0.133 & 0.403 & 0.958 & 1.767 & 2.76 & 3.715 & -0.15 & -0.57 & -1.09 & -1.49 & -1.77 & -1.94 \\
\hline 5 & 0.261 & 0.698 & 1.629 & 2.986 & 4.677 & 6.303 & -0.10 & -0.96 & -1.67 & -2.11 & -2.75 & -2.80 \\
\hline 6 & 0.291 & 0.802 & 1.876 & 3.430 & 5.365 & 7.230 & -0.14 & -0.95 & -1.83 & -2.53 & -3.10 & -3.73 \\
\hline
\end{tabular}

Table 5 - FLAP0 Measured Bending Deflection $W_{i}$ (inches) and Twist about Pitch Axis $\Delta \alpha_{i}(\mathrm{deg}), i=1, \ldots, 6$

The measured twist is about the wing pitch axis that includes both the torsional and bending deflections according to

$$
\Delta \alpha_{i}=-\Theta_{i} \cos \Lambda-W_{i} \sin \Lambda
$$

To estimate the static mode shapes, the elastic axis definition needs to be established. Based on the NASTRAN beam-stick model generated by UWAL presented in Ref., ${ }^{9}$ the equivalent elastic axis is estimated to be approximately at the $40 \%$ chord location of the wing root section with a sweep angle of $20.83^{\circ}$. A linear regression is then performed using the first six bending mode shapes to compute the generalized displacements

$$
w=\left[\sum_{i=1}^{n} \Phi^{\top}\left(\bar{y}_{i}\right) \Phi\left(\bar{y}_{i}\right)\right]^{-1} \sum_{i=1}^{n} \Phi^{\top}\left(\bar{y}_{i}\right) W_{i}
$$

These generalized displacements are plotted in Fig. 63 as functions of the angle of attack.

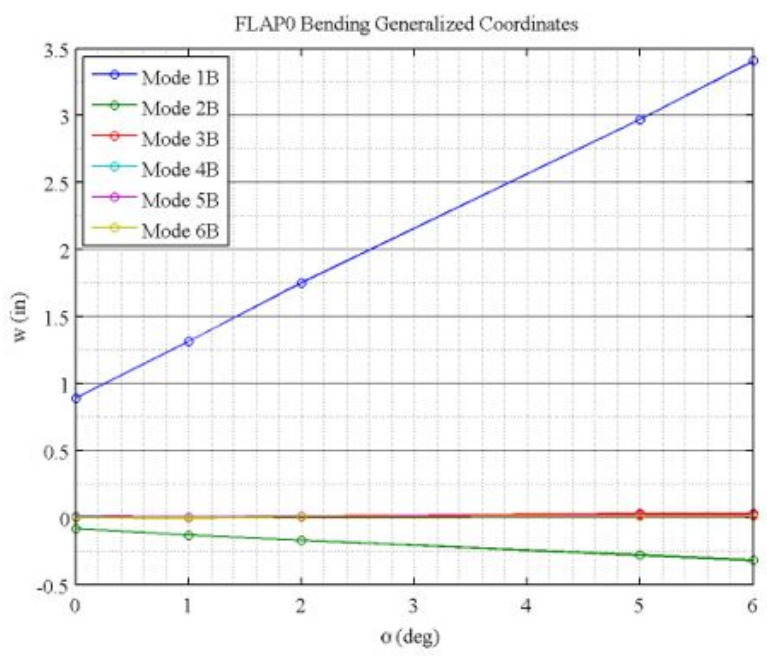

Fig. 63 - FLAP0 Bending Generalized Displacements 
The results show a linear variation of the generalized coordinates with respect to the angle of attack. The two significant modes that contribute to the static bending mode shapes are the first bending mode (1B) and the second bending mode (2B). Therefore, we can express

$$
w=w_{0}+\frac{\partial w}{\partial \alpha} \alpha
$$

The static bending mode shapes can be re-constructed from the generalized displacements, as shown in Fig. 64. The wing bending slopes then can be estimated readily from the static bending mode shapes, as shown in Fig. 65 .

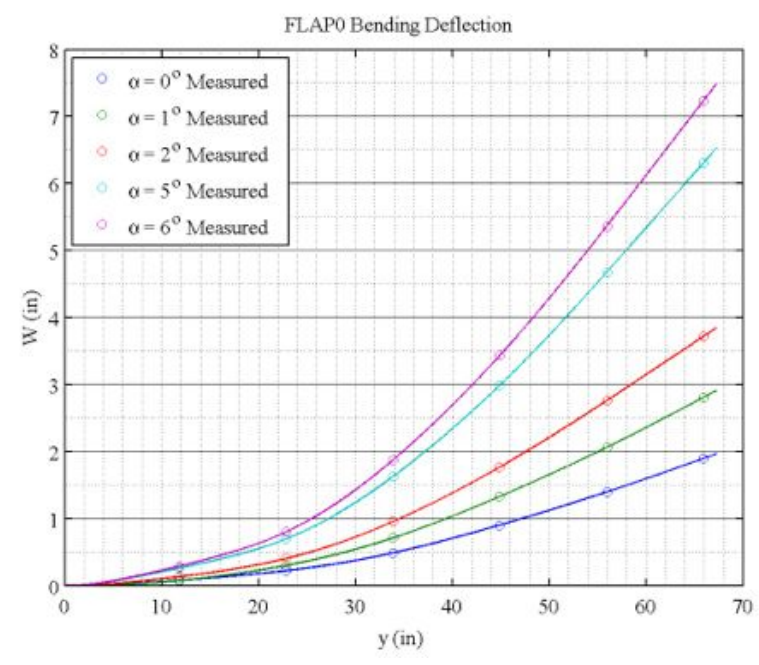

Fig. 64 - FLAP0 Bending Deflection

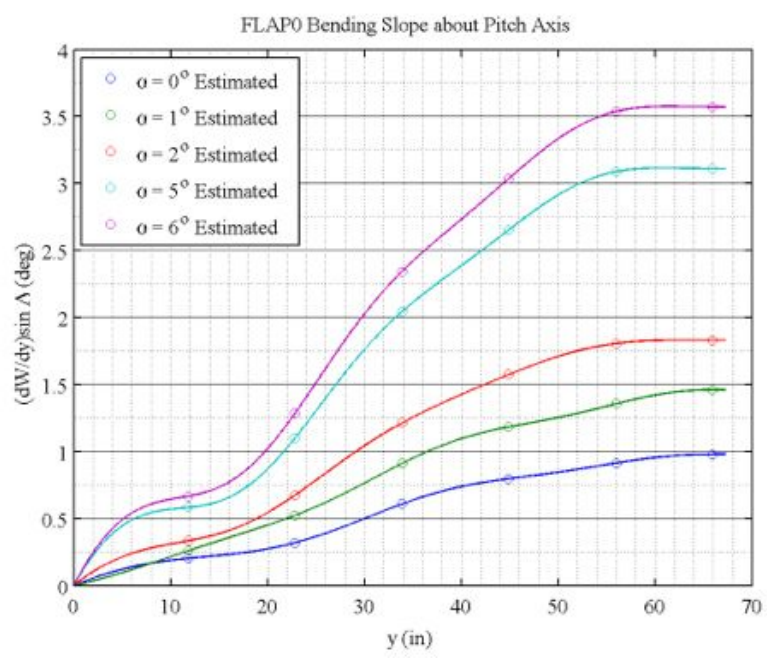

Fig. 65 - FLAP0 Bending Slope about Pitch Axis

The torsional deflections at the measurement locations about the elastic axis can be computed by

$$
\Theta_{i}=-\frac{\Delta \alpha_{i}}{\cos \Lambda}-\frac{d W_{i}}{d \bar{y}} \tan \Lambda
$$

The results are shown in Table 6. The torsional deflections are observed to be quite small. Thus, it is evident that bending deflection contributes primarily to the sectional angle of attack changes.

\begin{tabular}{|c|c|c|c|c|c|c|c|c|c|c|c|c|}
\hline$\alpha$ & $d W_{1} / d \bar{y}$ & $d W_{2} / d \bar{y}$ & $d W_{3} / d \bar{y}$ & $d W_{4} / d \bar{y}$ & $d W_{5} / d \bar{y}$ & $d W_{6} / d \bar{y}$ & $\Theta_{1}$ & $\Theta_{2}$ & $\Theta_{3}$ & $\Delta \alpha_{4}$ & $\Delta \alpha_{5}$ & $\Delta \alpha_{6}$ \\
\hline \hline 0 & 0.571 & 0.900 & 1.715 & 2.242 & 2.569 & 2.755 & -0.174 & -0.085 & 0.075 & 0.046 & 0.114 & 0.268 \\
\hline 1 & 0.732 & 1.476 & 2.565 & 3.328 & 3.812 & 4.107 & -0.107 & -0.048 & -0.045 & -0.250 & -0.006 & -0.311 \\
\hline 2 & 0.940 & 1.897 & 3.414 & 4.429 & 5.079 & 5.157 & -0.197 & -0.112 & -0.133 & -0.091 & -0.039 & 0.114 \\
\hline 5 & 1.638 & 3.096 & 5.750 & 7.461 & 8.687 & 8.752 & -0.516 & -0.151 & -0.401 & -0.581 & -0.363 & -0.334 \\
\hline 6 & 1.877 & 3.614 & 6.595 & 8.537 & 9.950 & 10.047 & -0.564 & -0.358 & -0.551 & -0.541 & -0.469 & 0.168 \\
\hline
\end{tabular}

Table 6 - FLAP0 Calculated Bending Slope $d W_{i} / d \bar{y}$ (inches) and Torsional Deflection about Elastic Axis $\Theta_{i}(\operatorname{deg}), i=1, \ldots, 6$

The torsional generalized rotations are computed and plotted in Fig. 66 as functions of the angle of attack. Since the torsional deflections are quite small, there is a considerable scatter in the data. The static torsional mode shapes are shown in Fig. 67. These mode shapes do not exhibit well-behaved trends due to small amplitudes in the torsional deflections.

The rigid-wing lift coefficient can now be estimated from the static bending and torsional mode shapes of the wing. The wing aeroelastic deflection results in an effective change in the angle of attack, which is computed as

$$
\Delta \alpha=-\frac{1}{C_{L_{\alpha}}^{*} S} \int_{-b / 2}^{b / 2} c_{L_{\alpha}}^{*}(y)\left[\Psi(\bar{y}) \theta \cos \Lambda+\frac{d \Phi(\bar{y})}{d \bar{y}} w \sin \Lambda\right] c(y) d y
$$

where $c_{L_{\alpha}}^{*}(y)$ is the sectional lift curve slope for the rigid wing.

Thus, to estimate the effective change in the angle of attack, we need to have a knowledge of the sectional lift curve slope, which could only be estimated from an aerodynamic model. Absent of this information, we can assume that the sectional lift 
curve slope is the same as the wing lift curve slope. Then, by this assumption, the effective change in the angle of attack can be computed as

$$
\Delta \alpha=-\frac{1}{S} \int_{-b / 2}^{b / 2}\left[\Psi(\bar{y}) \theta \cos \Lambda+\frac{d \Phi(\bar{y})}{d \bar{y}} w \sin \Lambda\right] c(y) d y
$$

This effective change in the angle of attack is a function of the angle of attack as

$$
\Delta \alpha=\Delta \alpha_{0}+\frac{\partial \Delta \alpha}{\partial \alpha} \alpha
$$

which is shown in Fig. 68.

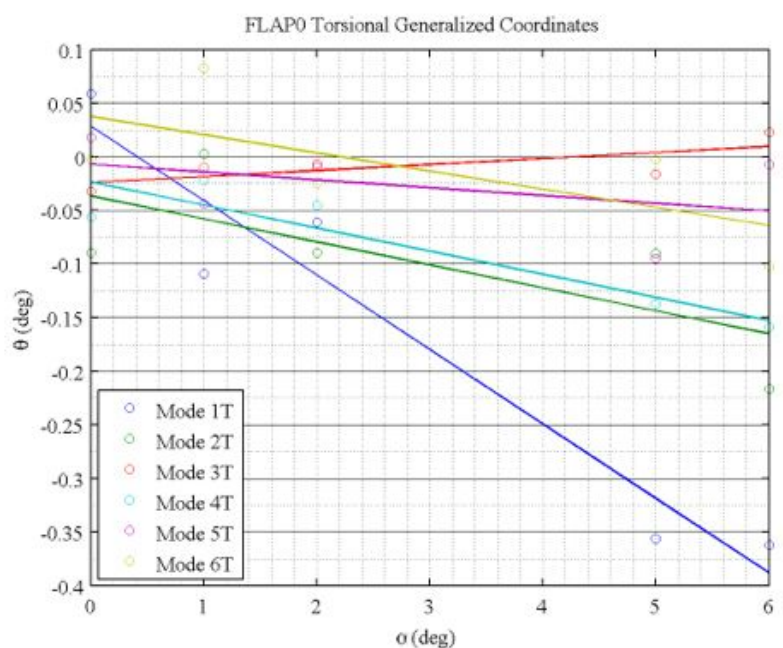

Fig. 66 - FLAP0 Torsional Generalized Rotations

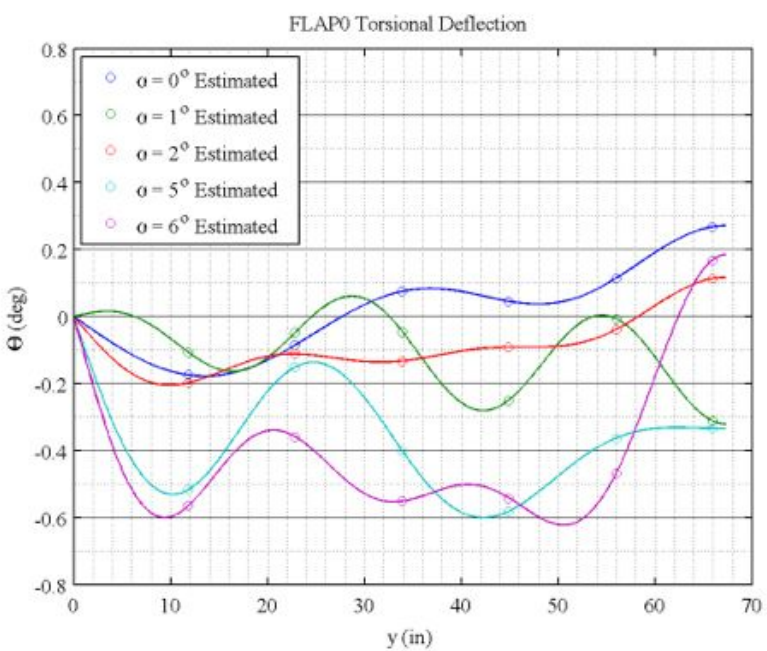

Fig. 67 - FLAP0 Torsional Deflection

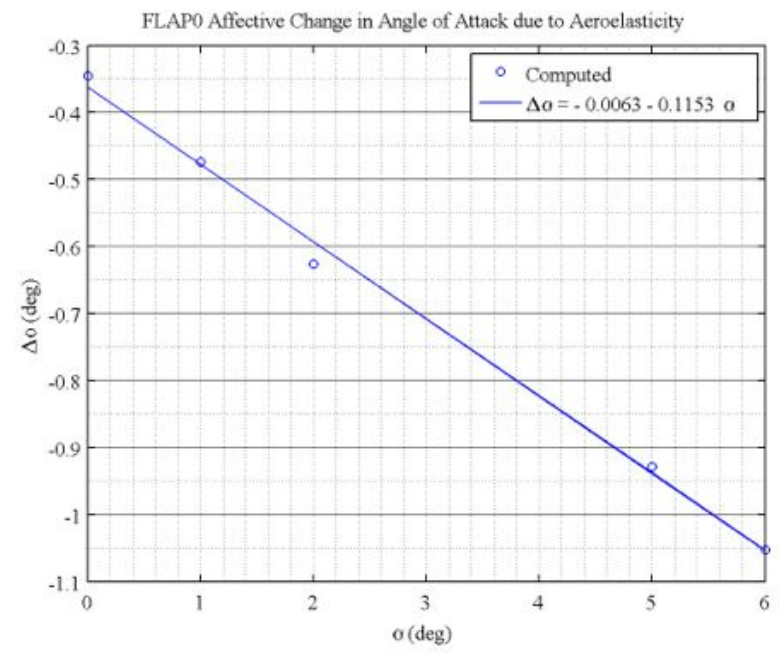

Fig. 68 - FLAP0 Change in Angle of Attack due to Aeroelasticity

From Eqs. (52) and (53), we can write

$$
\begin{gathered}
C_{L_{0}}=C_{L_{0}}^{*}+C_{L_{\alpha}}^{*} \Delta \alpha_{0} \\
C_{L_{\alpha}}=C_{L_{\alpha}}^{*}+C_{L_{\alpha}}^{*} \frac{\partial \Delta \alpha}{\partial \alpha}
\end{gathered}
$$

Solving for $C_{L_{0}}^{*}$ and $C_{L_{\alpha}}^{*}$ yields

$$
\begin{aligned}
C_{L_{0}}^{*} & =C_{L_{0}}-\frac{C_{L_{\alpha}} \Delta \alpha_{0}}{1+\frac{\partial \Delta \alpha}{\partial \alpha}} \\
C_{L_{\alpha}}^{*} & =\frac{C_{L_{\alpha}}}{1+\frac{\partial \Delta \alpha}{\partial \alpha}}
\end{aligned}
$$


Thus, the rigid-wing lift coefficient is computed from the flexible-wing lift coefficient as

$$
C_{L}^{*}=C_{L}-\frac{C_{L_{\alpha}} \Delta \alpha}{1+\frac{\partial \Delta \alpha}{\partial \alpha}}
$$

Therefore, the rigid-wing lift coefficient for FLAP0 configuration from Fig. 18 is obtained as

$$
C_{L}^{*}=0.2074+4.6889 \alpha
$$

Assuming that the aeroelastic contribution to the lift coefficient for FLAP0 configuration from run 116 to 120 holds for the data from runs $18,19,20,23$, and 24 in light of the different lift curves between these two data sets, the rigid-wing lift coefficient expression can also be estimated by the $q_{\infty}$-correction method using the cubic variation for $C_{L}$ at $q_{\infty}=20 \mathrm{psf}$ as

$$
\begin{aligned}
C_{L}^{*} & =0.1778+4.1482 \alpha-\left(1.8731 \times 10^{-7} q_{\infty}^{3}-1.9561 \times 10^{-5} q_{\infty}^{2}-6.1839 \times 10^{-4} q_{\infty}\right) \\
& -\left(-2.2077 \times 10^{-5} q_{\infty}^{3}+1.3363 \times 10^{-3} q_{\infty}^{2}-3.8151 \times 10^{-2} q_{\infty}\right) \alpha=0.1965+4.5533 \alpha
\end{aligned}
$$

Alternatively, using the linear variation for $C_{L}$ at $q_{\infty}=20 \mathrm{psf}$, the rigid-wing lift coefficient for FLAP0 configuration is estimated to be

$$
\begin{aligned}
C_{L}^{*} & =0.1778+4.1482 \alpha-\left(-1.1601 \times 10^{-3}-1.3068 \times 10^{-2} \alpha\right) q_{\infty} \\
& =0.2010+4.4096 \alpha
\end{aligned}
$$

Figure 69 shows the rigid-wing lift coefficients estimated by the aeroelastic deflection correction and the two $q_{\infty}$-correction methods as compared to the flexible-wing lift coefficient. The aeroelastic deflection correction method yields the highest estimated rigid-wing lift coefficient. Since there are some differences in the three estimated rigid-wing lift coefficients, an average rigid-wing lift coefficient is computed and is shown in Fig. 69.

Both the aeroelastic deflection correction method and the $q_{\infty}$-correction method exhibit some degree of uncertainty. For the aeroelastic deflection correction method, the assumption of the sectional lift curve slope being the same as the wing lift curve slope is made. This assumption needs to be verified by aerodynamic analysis using CFD. Also, the aeroelastic deflection measurements have uncertainty involved with the accuracy of the VICON system. This uncertainty can be quantified by an uncertainty quantification analysis which is not within the scope of this study.

For the $q_{\infty}$-correction method, data extrapolation is usually not considered to be a reliable experimental data analysis technique. Depending on the type of regressors such as the cubic and linear functions, the results can differ. Thus, the estimation of the rigid-wing lift coefficient is subject to some degree of uncertainty. The average rigid-wing lift coefficient therefore represents the best estimate of the uncertain estimation of the three sets of rigid-wing lift coefficients.

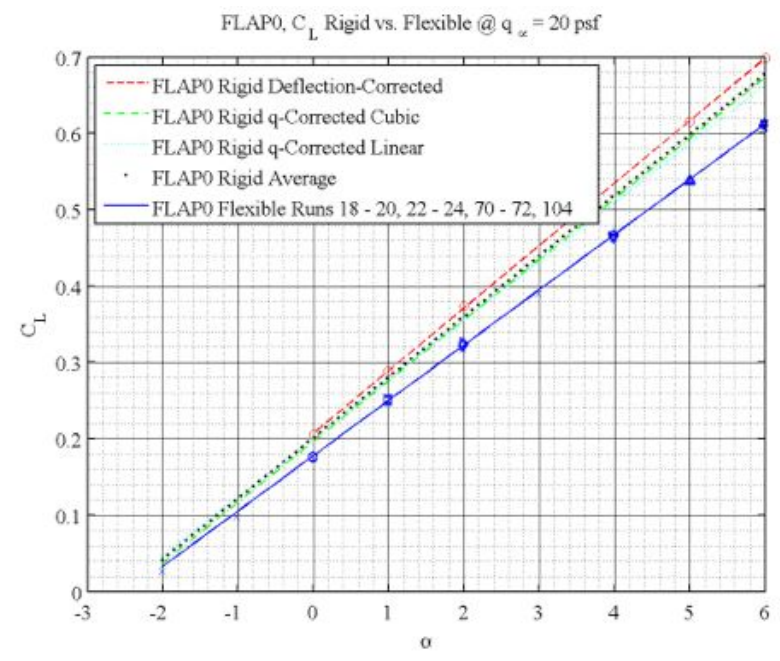

Fig. 69 - FLAP0 Runs 18 - 20, 22 - 24, 70 - 72, $104 C_{L}$ of Flexible and Rigid Wing

Using this approach, we can estimate the rigid-wing lift coefficients for the other VCCTEF configurations for which aeroelastic deflection measurements are available. FLAP7 configuration is examined next for comparison to the estimated rigid-wing lift coefficient computed by the $q_{\infty}$-correction method. Figures 70 and 71 are the plots of the static bending mode shape and 
the estimated rigid-wing lift curves of FLAP7 configuration. The rigid-wing lift coefficient computed by the $q_{\infty}-$ correction method using the cubic variation of $C_{L}$ with $q_{\infty}$ is closer to the rigid-wing lift coefficient computed by the aeroelastic deflection correction method than that computed by the $q_{\infty}$-correction method using the linear variation. The results indicate a higher uncertainty for FLAP7 configuration which mainly is to due to the linear and cubic data extrapolations used in the $q_{\infty}$-correction method. The results also seem to suggest that the linear variation of $C_{L}$ with $q_{\infty}$ is perhaps less accurate than the cubic variation. This is consistent with the theory developed in the aeroelastic analysis which shows that the aeroelastic effect on the lift coefficient is described by a higher-degree polynomial in $q_{\infty}$. If the estimated rigid-wing lift coefficient using the linear variation is not included, then the estimates obtained from the aeroelastic deflection correction method and the $q_{\infty}$-correction method are reasonably consistent. The average estimated rigid-wing lift coefficient is also computed and plotted in Fig. 71.

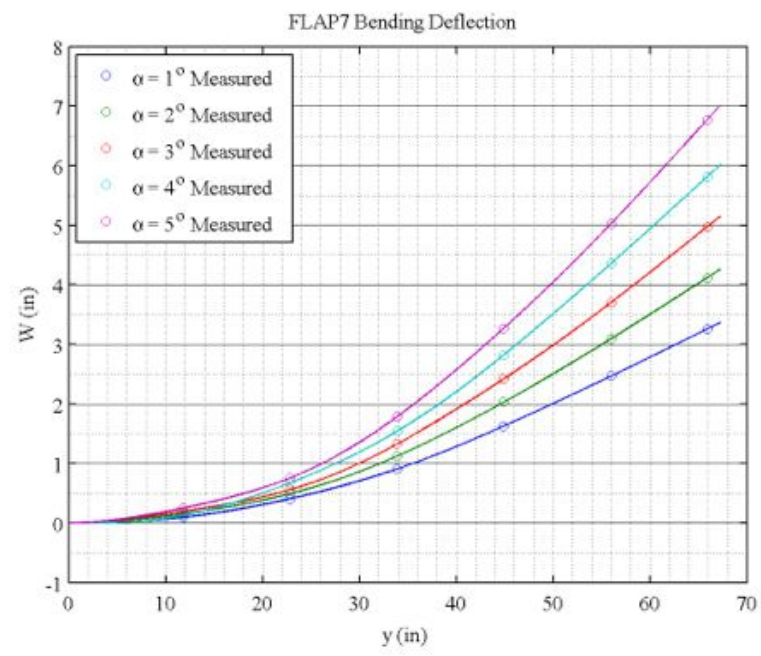

Fig. 70 - FLAP7 Bending Deflection

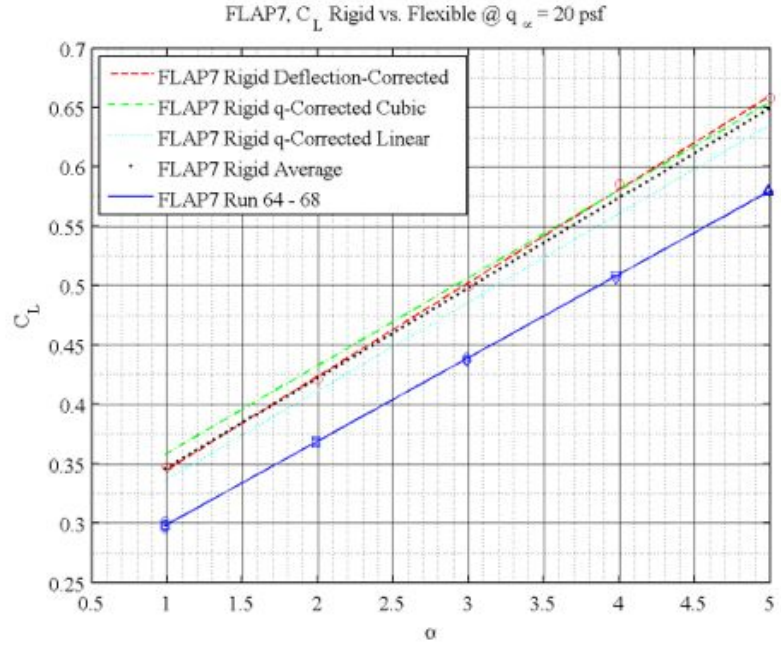

Fig. 71 - FLAP7 Runs 68 - $68 C_{L}$ of Flexible and Rigid Wing

Figures 72 to 89 show the rigid-wing lift coefficients of all the other VCCTEF configurations computed by the aeroelastic deflection correction method. It can be seen that the aeroelastic deflections can cause a significant loss of lift for high-lift configurations such as FLAP1 configuration. There is a clear trend that, as the incremental lift increases due to the VCCTEF deflections, the difference between the rigid-wing lift coefficient and the flexible-wing lift coefficients also increases.

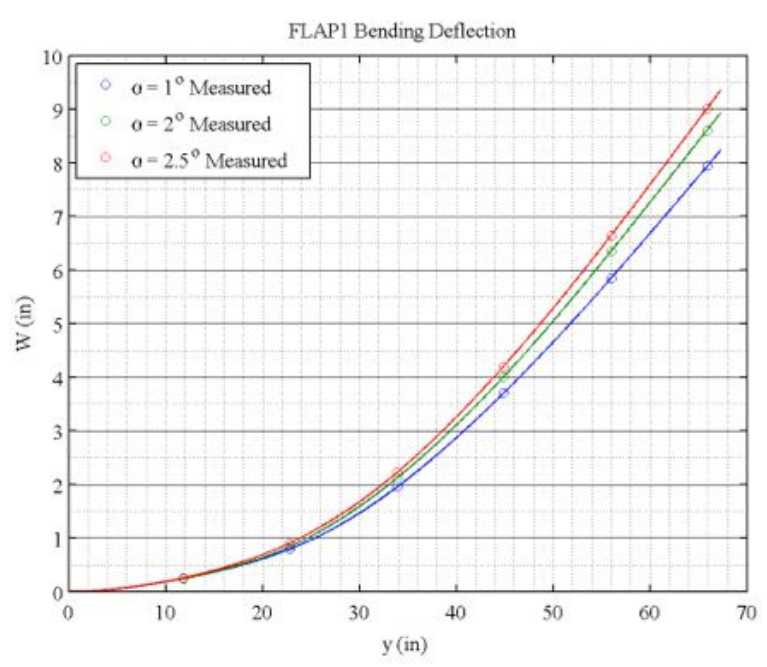

Fig. 72 - FLAP1 Bending Deflection

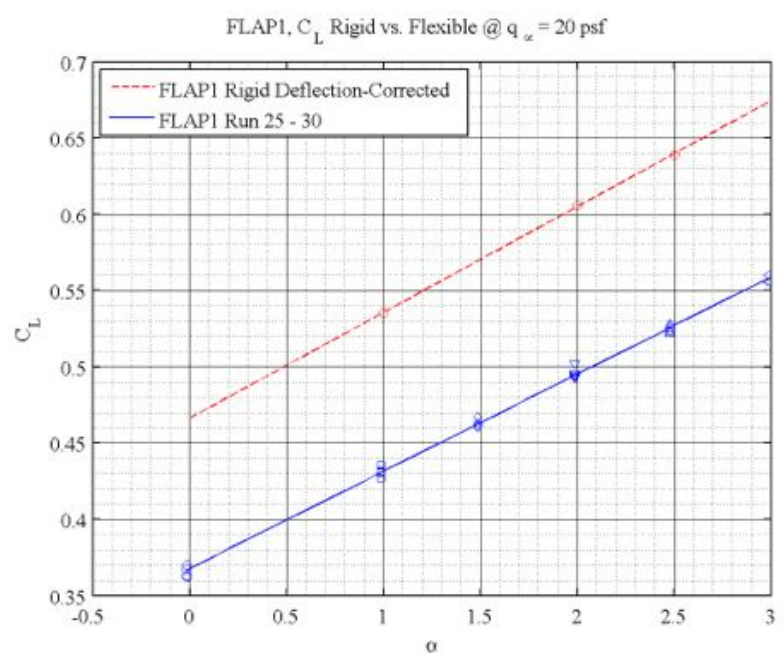

Fig. 73 - FLAP1 Runs $25-30 C_{L}$ of Flexible and Rigid Wing 


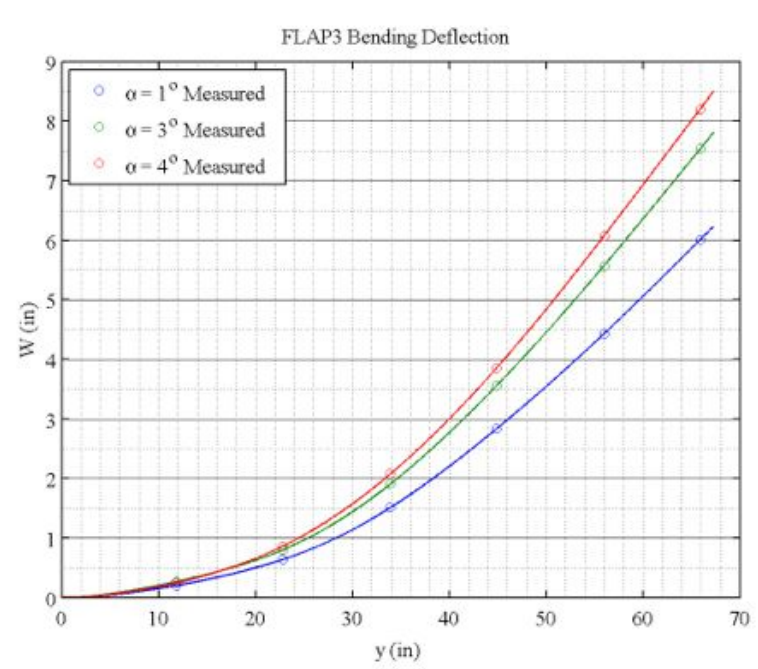

Fig. 74 - FLAP3 Bending Deflection

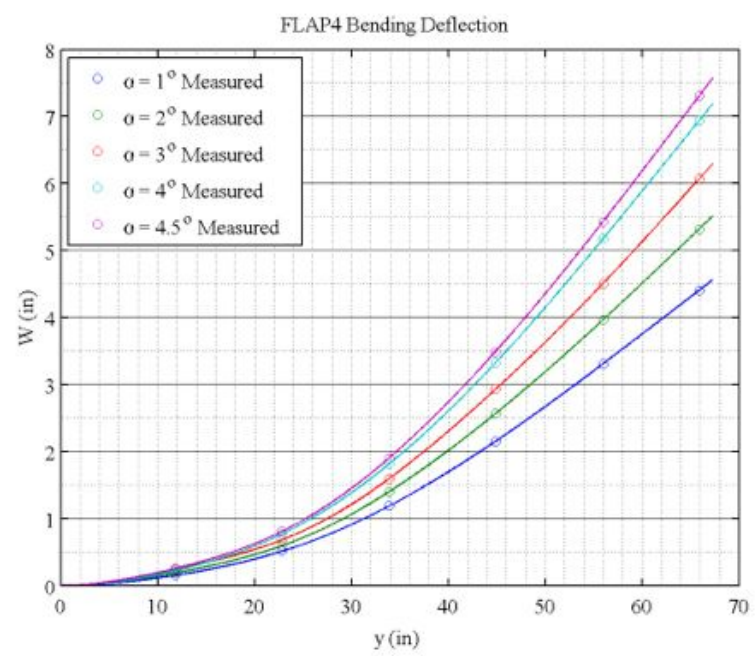

Fig. 76 - FLAP4 Bending Deflection

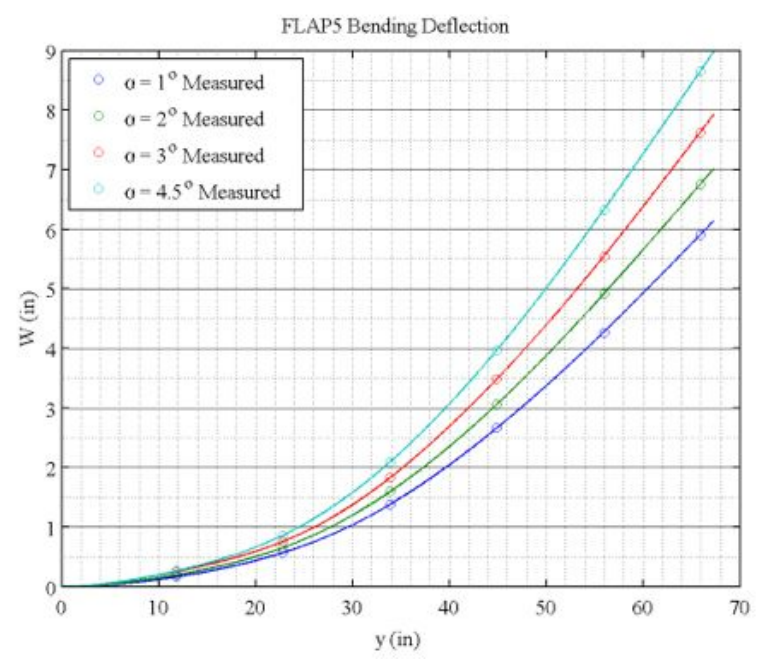

Fig. 78 - FLAP5 Bending Deflection

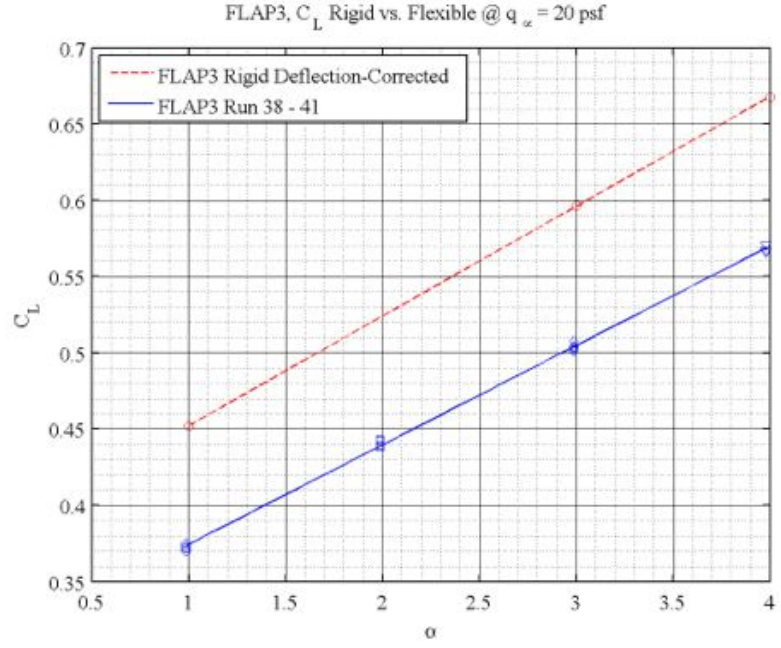

Fig. 75 - FLAP3 Runs $38-41 C_{L}$ of Flexible and Rigid Wing

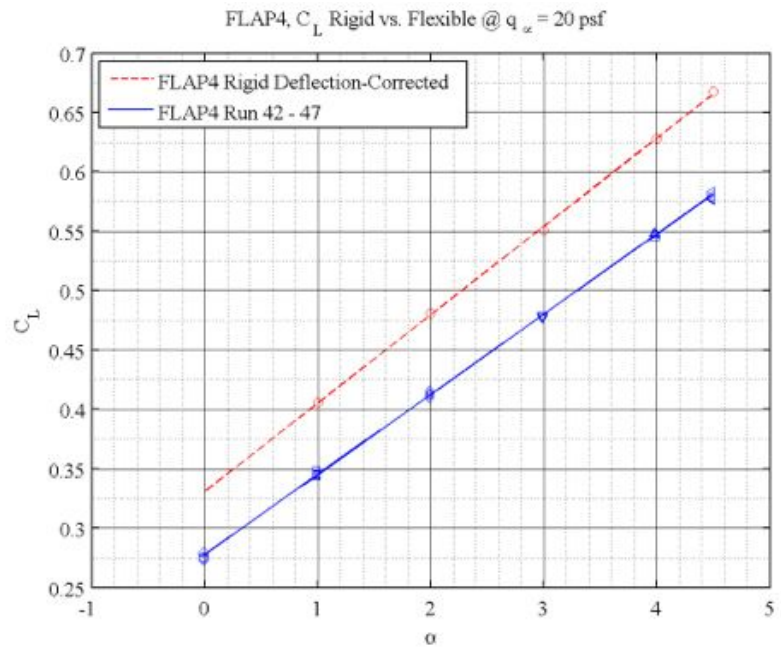

Fig. 77 - FLAP4 Runs $42-47 C_{L}$ of Flexible and Rigid Wing

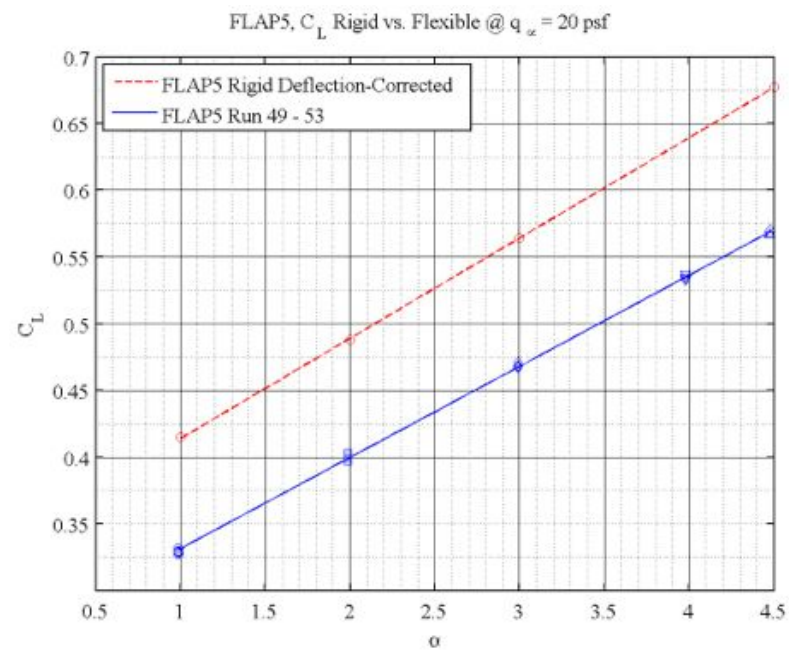

Fig. 79 - FLAP5 Runs $49-53 C_{L}$ of Flexible and Rigid Wing 


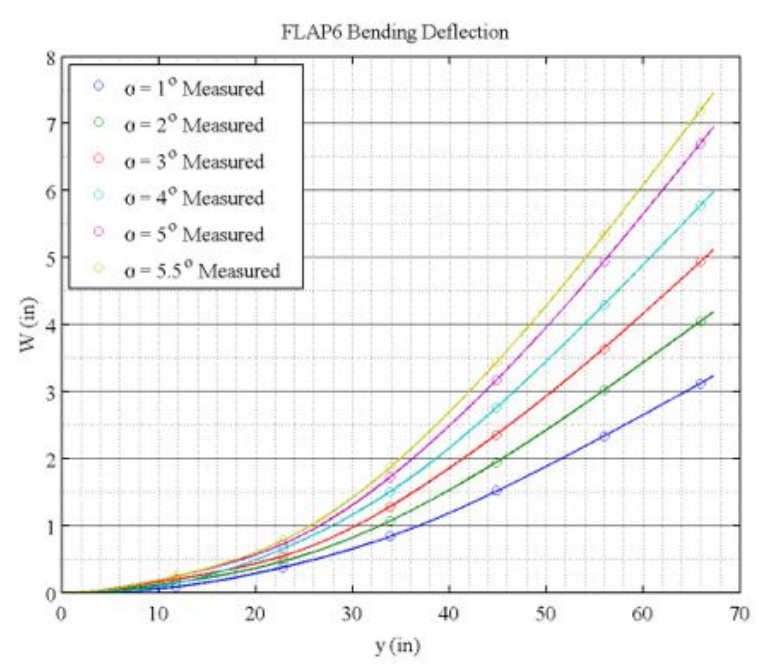

Fig. 80 - FLAP6 Bending Deflection

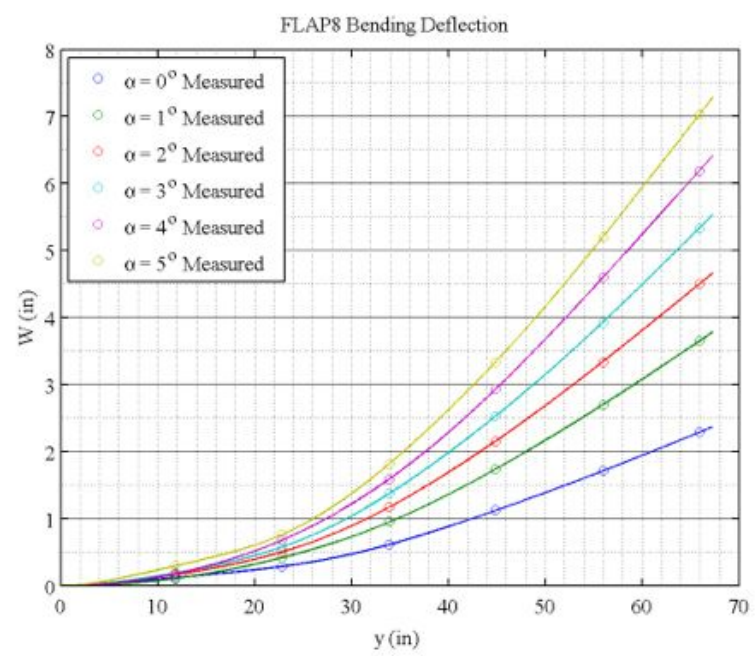

Fig. 82 - FLAP8 Bending Deflection

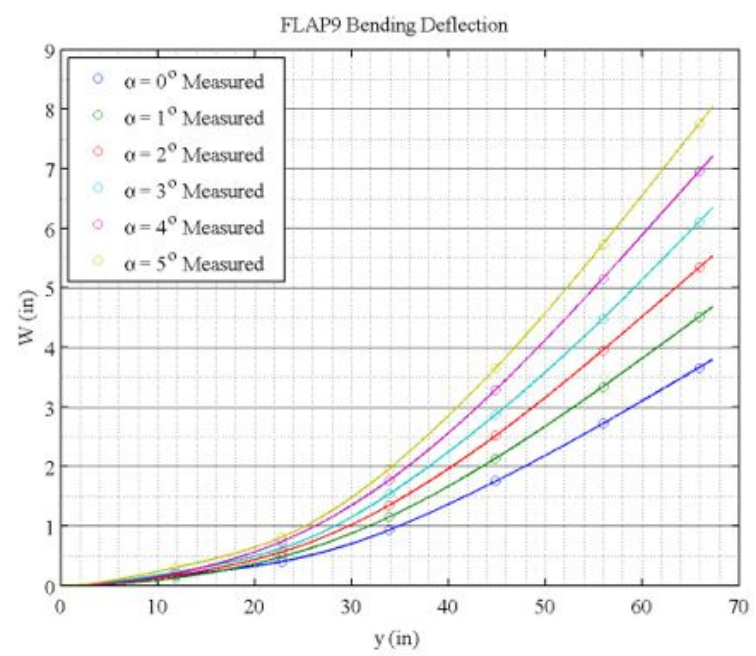

Fig. 84 - FLAP9 Bending Deflection
FLAP6, C Rigid vs. Flexible @ $\mathrm{q}_{\alpha}=20 \mathrm{psf}$

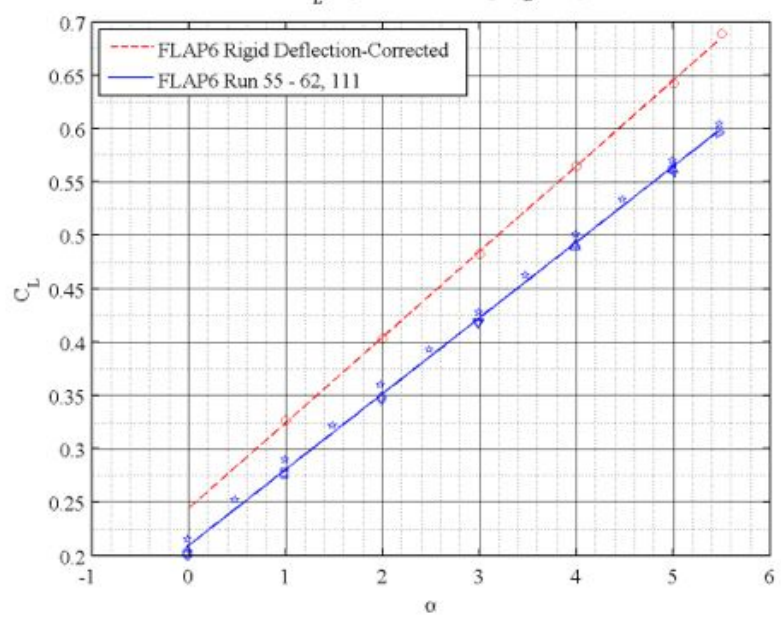

Fig. 81 - FLAP6 Runs 55 - 62, $111 C_{L}$ of Flexible and Rigid Wing

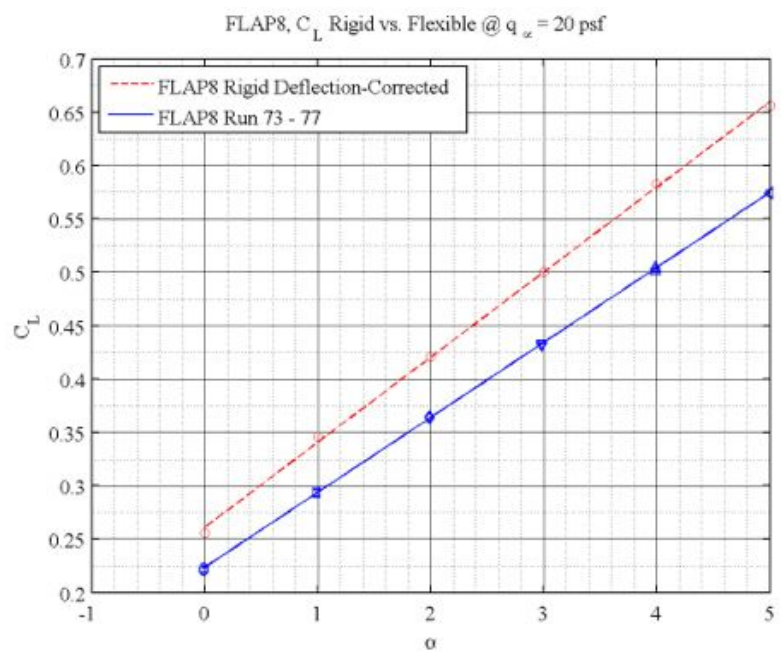

Fig. 83 - FLAP8 Runs 73 - $77 C_{L}$ of Flexible and Rigid Wing

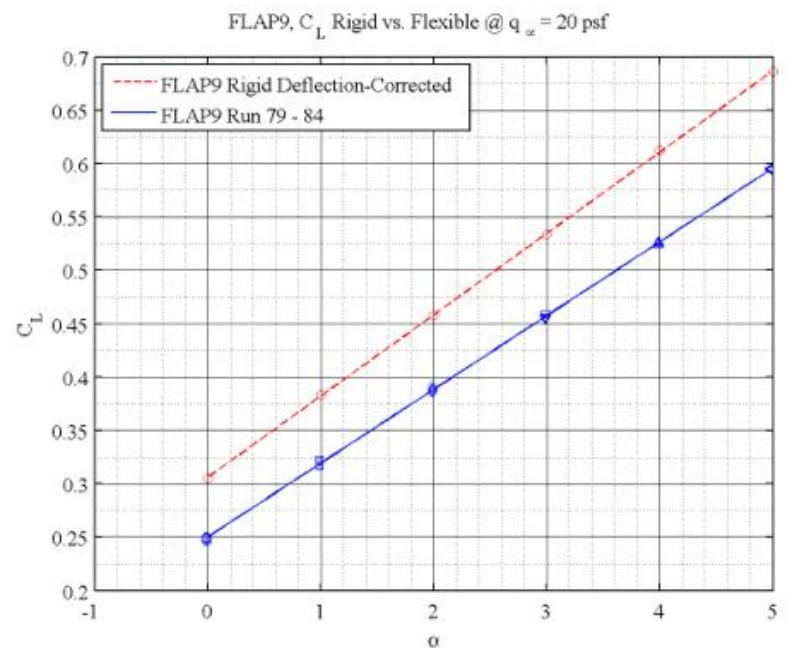

Fig. 85 - FLAP9 Runs $79-84 C_{L}$ of Flexible and Rigid Wing 


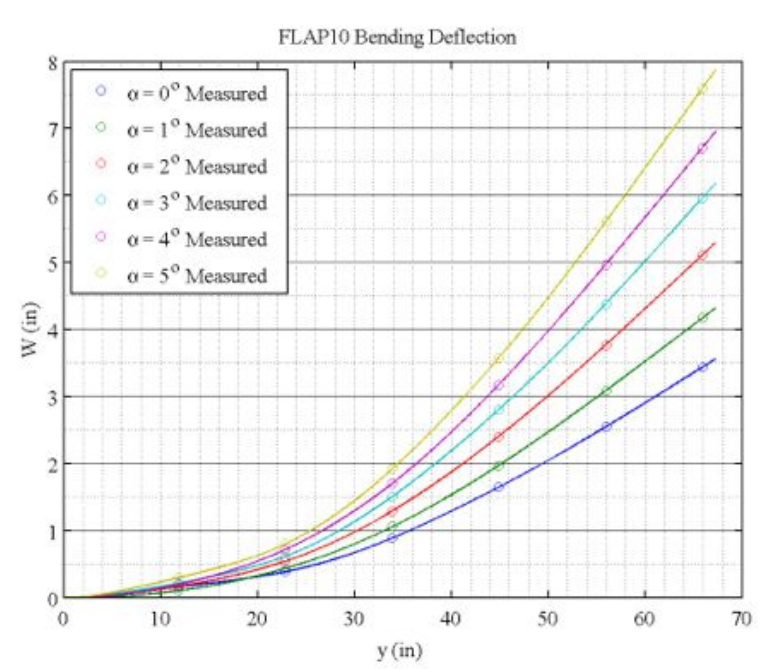

Fig. 86 - FLAP10 Bending Deflection

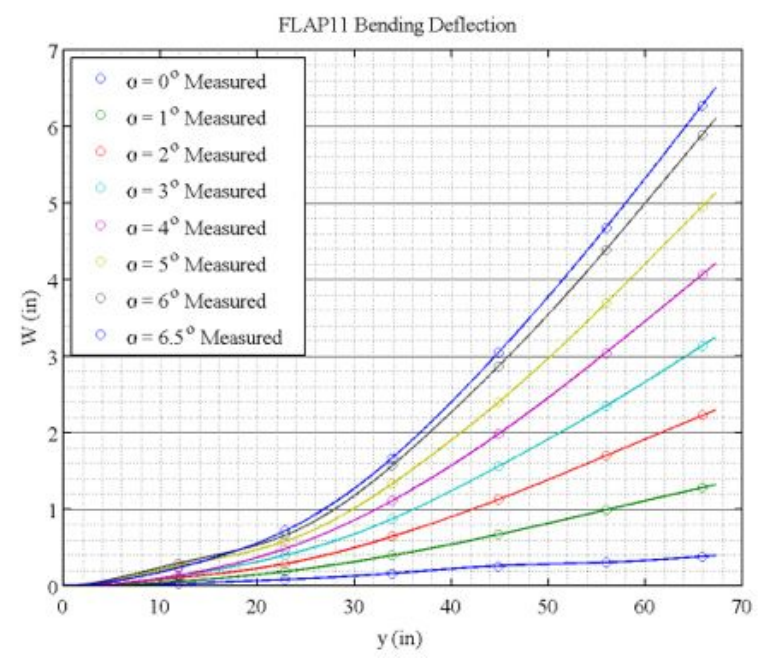

Fig. 88 - FLAP11 Bending Deflection

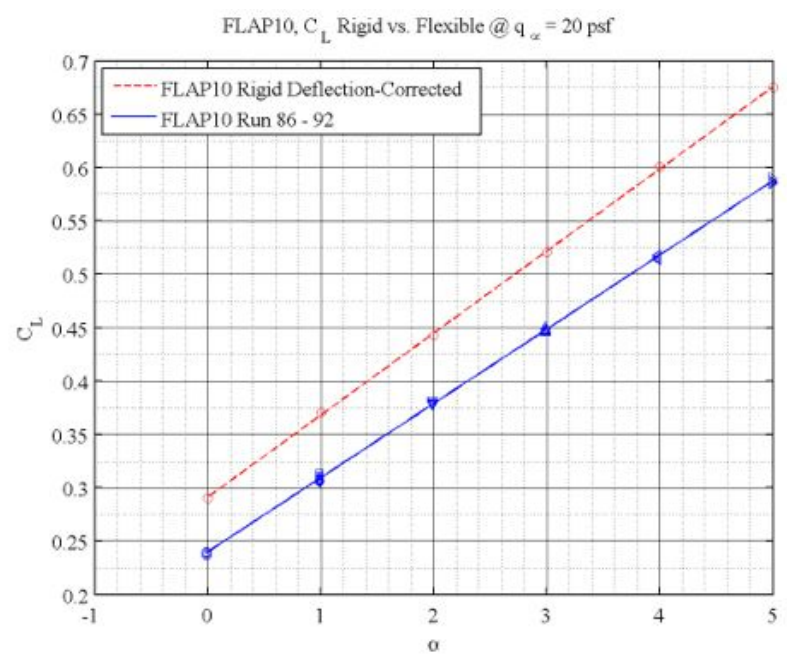

Fig. 87 - FLAP10 Runs $79-84 C_{L}$ of Flexible and Rigid Wing

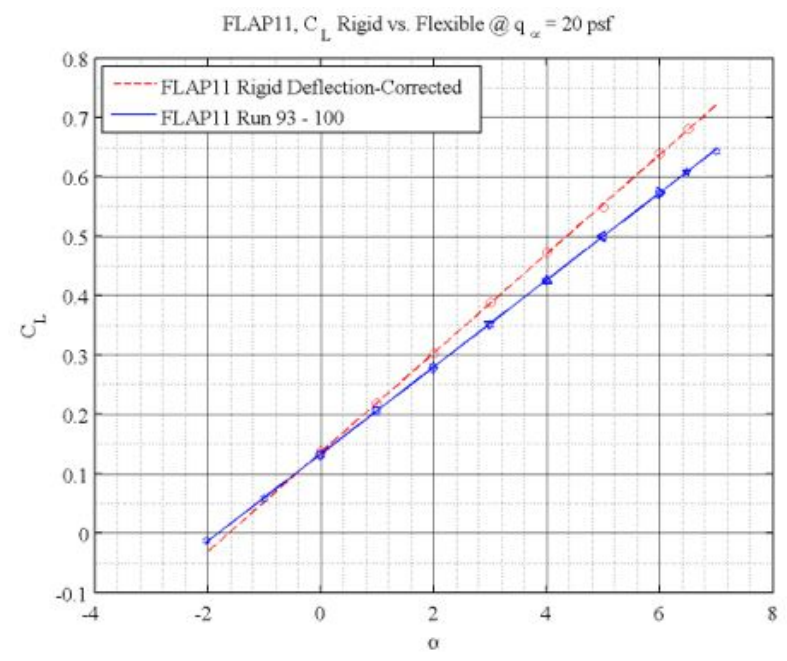

Fig. 89 - FLAP11 Runs $93-100 C_{L}$ of Flexible and Rigid Wing

\section{Conclusion}

This paper presents experimental results of a flexible wing wind tunnel model with a variable camber continuous trailing edge flap (VCCTEF) design for drag minimization tested at the University of Washington Aeronautical Laboratory (UWAL). The wind tunnel test was designed to explore the relative merit of the VCCTEF concept for improved cruise efficiency and the use of low-cost aeroelastic model test techniques. The flexible wing model is a $10 \%$-scaled model of a typical transport wing and is constructed of woven fabric composites and foam core. The wing structural stiffness in bending is tailored to be half the stiffness of a Boeing 757-era transport wing while the torsional stiffness is about the same. This stiffness reduction results in a wing tip deflection of about $10 \%$ of the wing semi-span. The VCCTEF is a multi-segment flap design having three chordwise camber segments and five spanwise flap segments for a total of 15 flap segments. The three chordwise camber segments can be positioned appropriately to create a desired trailing edge camber. Elastomeric material is used to cover the gaps in between the spanwise flap segments, thereby creating a continuous trailing edge. Wind tunnel data indicate a high degree of data correlation and repeatability. The VCCTEF can achieve a drag reduction of up to $6.31 \%$ and an improvement in $L / D$ of up to $4.85 \%$. These improvements in $L / D$ are applicable to low-speed incompressible flow for a wing-body configuration. Further experimental investigations will be needed to fully assess the performance of the VCCTEF for a full configuration in high-speed flow. The paper also presents two methods for estimating the lift coefficient of the rigid wing using a dynamic pressure correction and an aeroelastic deflection correction. Both methods provide good estimates of the rigid-wing lift coefficient, although they both 
have some degree of uncertainty. Aerodynamic modeling will be conducted in the future to refine the estimates of the rigid-wing lift coefficient presented in this study.

\section{Acknowledgment}

The authors would like to thank the Fixed Wing Project under the Fundamental Aeronautics Program of NASA Aeronautics Research Mission Directorate (ARMD) for funding support of this work. The authors also would like to acknowledge Boeing Research and Technology and the University of Washington for their collaboration with NASA under NASA contract NNL11AA05B task order NNL11AD25T entitled "Development of Variable Camber Continuous Trailing Edge Flap System."

\section{References}

${ }^{1}$ Nguyen, N., "Elastically Shaped Future Air Vehicle Concept," NASA Innovation Fund Award 2010 Report, October 2010, Submitted to NASA Innovative Partnerships Program.

${ }^{2}$ Nguyen, N., Trinh, K., Reynolds, K., Kless, J., Aftosmis, M., Urnes, J., and Ippolito, C., "Elastically Shaped Wing Optimization and Aircraft Concept for Improved Cruise Efficiency," AIAA Aerospace Sciences Meeting, AIAA-2013-0141, January 2013.

${ }^{3}$ Boeing Report No. 2012X0015, "Development of Variable Camber Continuous Trailing Edge Flap System," October 4, 2012.

${ }^{4}$ Urnes, J., Nguyen, N., Ippolito, C., Totah, J., Trinh, K., and Ting, E., “A Mission Adaptive Variable Camber Flap Control System to Optimize High Lift and Cruise Lift to Drag Ratios of Future N+3 Transport Aircraft," AIAA Aerospace Sciences Meeting, AIAA-2013-0214, January 2013.

${ }^{5}$ Jordan, T. L., Langford, W. M., Belcastro, C. M., Foster, J. M., Shah, G. H., Howland, G., and Kidd, R., "Development of a Dynamically Scaled Generic Transport Model Testbed for Flight Research Experiments," AUVSI Unmanned Unlimited, Arlington, VA, 2004

${ }^{6}$ Bisplinghoff, R. L, Ashley, H., and Halfman, R. L, Aeroelasticity, Addison-Wesley, 1955, pp. 478 - 480.

${ }^{7}$ Anderson, J. D., Fundamentals of Aerodynamics, McGraw-Hill, 2001, pp. 289 - 310.

${ }^{8}$ Hodges, D. and Pierce, G., Introduction to Structural Dynamics and Aeroelasticity, Cambridge University Press, 2002.

${ }^{9}$ Precup, N., Mor, M., and Livne, E., "Design, Construction, and Tests of an Aeroelastic Wind Tunnel Model of a Variable Camber Continuous Trailing Edge Flap (VCCTEF) Concept Wing," AIAA Aviation and Aeronautics Forum and Exposition, Atlanta, GA, June 2014.

${ }^{10}$ Rodriguez, D., Aftosmis, M., Nemec, M., and Smith, S., "Static Aeroelastic Analysis with an Inviscid Cartesian Method," AIAA Science and Technology Forum, AIAA-2014-0836, National Harbor, MD, January 2014. 